\title{
MODELOS UNIVARIADO E MULTIVARIADO PARA ANÁLISE DE MEDIDAS REPETIDAS E VERIFICAÇÃO DA ACURÁCIA DO MODELO UNIVARIADO POR MEIO DE SIMULAÇÃO
}

\section{LARA HOFFMANN XAVIER}

Bacharel em Estatística

Orientador:Prof Dr Carlos Tadeu dos Santos Dias

Dissertação apresentada à Escolā Superior de Agricultura "Luiz de Queiroz", Universidade de São Paulo, para obtenção do título de Mestre em Agronomia, Área de Concentração: Estatística e Experimentação Agronômica.

PIRACICABA

Estado de São Paulo - Brasil

Maio - 2000 


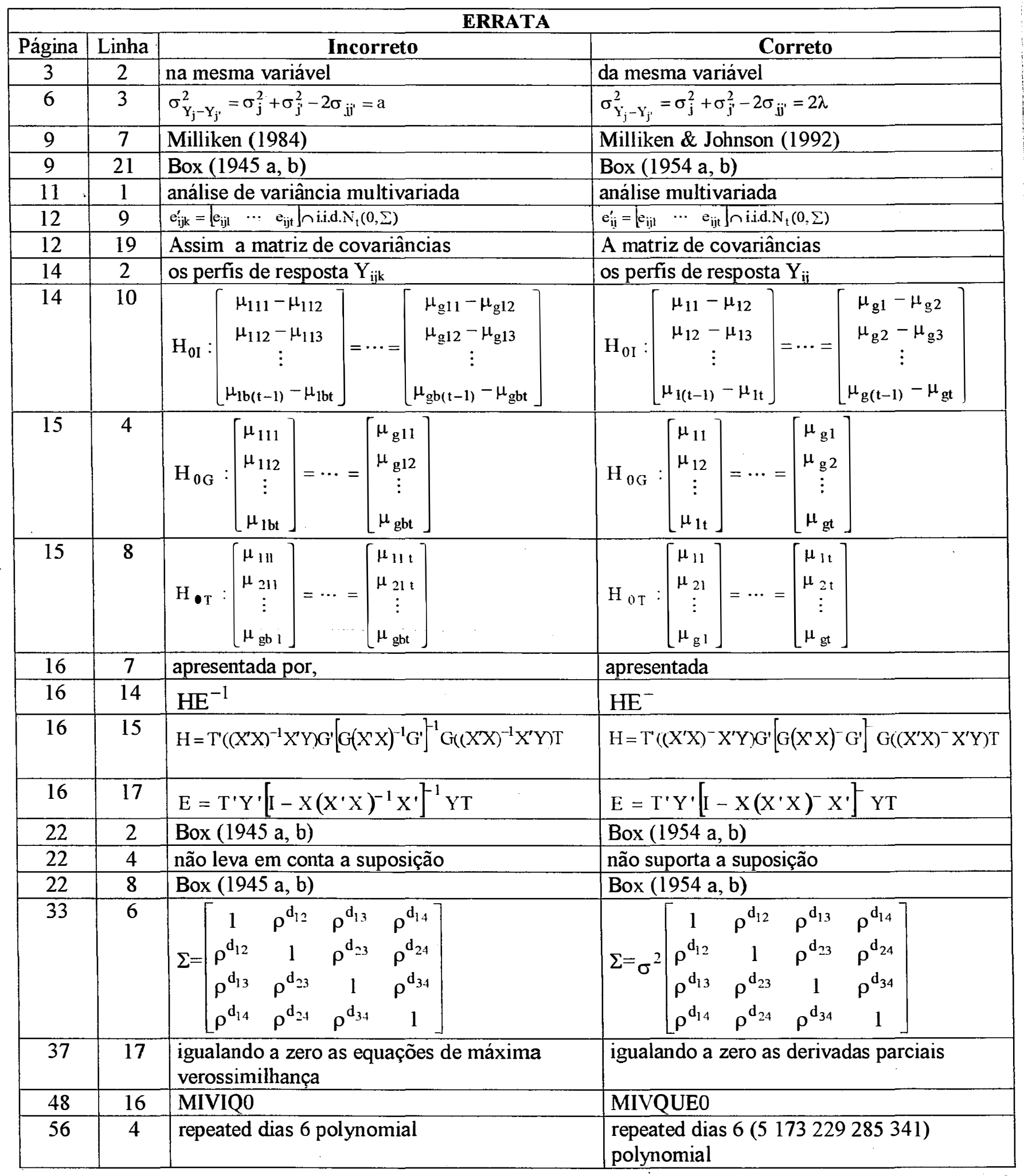


Dados Internacionais de Catalogação na Publicação (CIP)

DIVISÃO DE BIBLIOTECA E DOCUMENTAÇÃO - Campus “Luiz de Oueiroz"/USP

Xavier, Lara Hoffmann

Modelos univariado e multivariado para análise de medidas repetidas e verificação da acurácia do modelo univariado por meio de simulação / Lara Hoffmann Xavier. - Piracicaba, 2000.

$91 \mathrm{p}$.

Dissertação (mestrado) - - Escola Superior de Agricultura Luiz de Queiroz, 2000. Bibliografia.

1. Análise de covariância 2. Análise de variância 3. Estatística aplicada 4. Matriz de variância e covariância 5 . Método de máxima verossimilhança 6 . Método estatistico 7. Simulação I. Título

CDD 519.5

Permitida a copia total ou parcial deste documento, desde gue citada a fonte $=0$ antor? 
A Deus,
pela confiança e coragem.

Aos meus pais Nelsindo e Vanaeba,

pelo amor incondicional e incentivo, OFEREÇO.

Ao Almir, pela força, paciência e carinho, com muito amor, DEDICO. 


\section{AGRADECIMENTOS}

Ao Prof. Dr. Carlos Tadeu dos Santos Dias, pela amizade e orientação.

Aos professores do Departamento de Ciências Exatas da ESALQ/USP, principalmente à Prof ${ }^{a}$. Dr ${ }^{\mathrm{a}}$. Clarice Garcia Borges Demétrio, ao Prof. Dr. Décio Barbin, ao Prof. Dr. Antonio Francisco Iemma e ao Prof. Dr. Antonio Augusto Franco Garcia, pelos ensinamentos e amizade.

À CAPES - Coordenação de Aperfeiçoamento de Pessoal de Nível Superior, pelo apoio financeiro.

Aos funcionários do Departamento de Ciências Exatas da ESALQ/USP, pelo atendimento.

Aos colegas de curso, em especial ao Arlei pelo companheirismo e amizade.

A Rosa e a Diva, pelo carinho e amizade com que me acolheram.

A Iza, pela amizade, conselhos e ensinamentos.

Aos meus pais e irmão, por sempre acreditarem em mim.

Ao Almir, pela compreensão, confiança e incentivo constantes.

A todos aqueles que de alguma forma colaboraram para a realização deste trabalho. 


\section{SUMÁRIO}

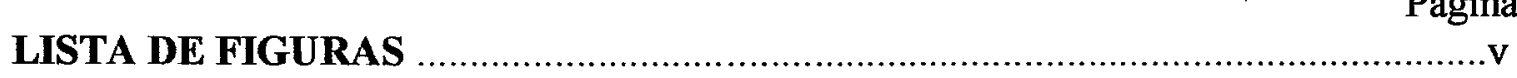

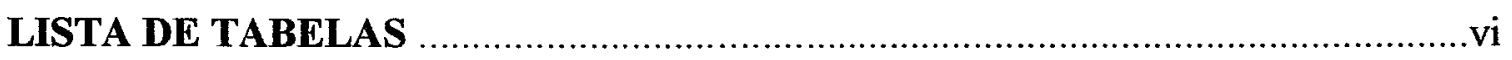

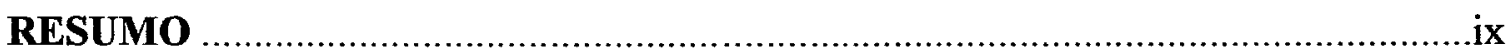

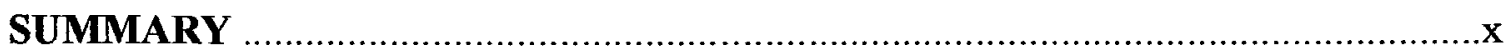

1 INTRODUÇ̃̃

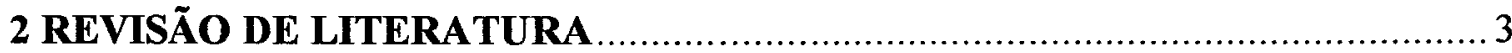

2.1 Teste de Esfericidade de Mauchly ........................................................... 7

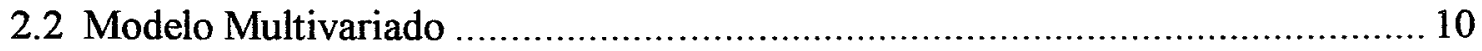

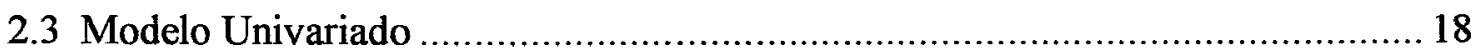

2.4 Correções para os Números de Graus de Liberdade .................................... 22

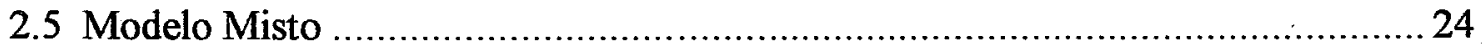

2.6 Estruturas da Matriz de Covariâncias ....................................................... 26

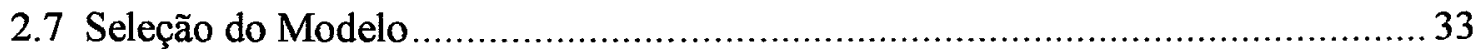

2.7.1 Teste Assintótico da Razão de Verossimilhança .................................. 34

2.7.2 Critério de Informação de Akaike (AIC) .......................................... 34

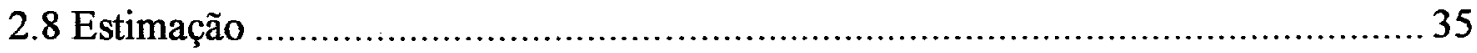

2.8.1 Método da Máxima Verossimilhança (MV) _...................................... 36

2.8.2 Método da Máxima Verossimilhança Restrita (MVR) ............................ 39

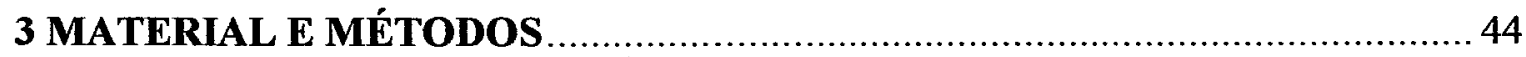

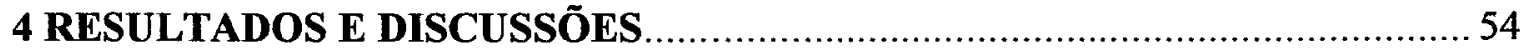

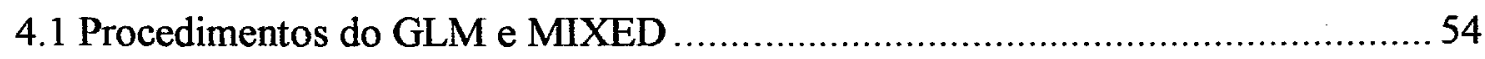

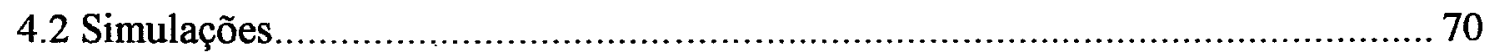

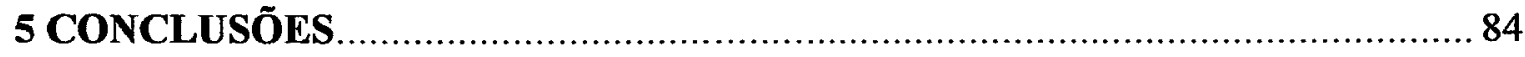

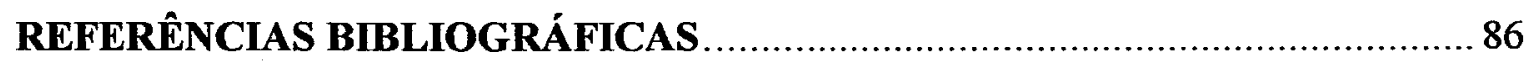




\section{LISTA DE FIGURAS}

Página

Figura 1. Diferenças verticais entre as curvas de tratamentos ...............................20

Figura 2. Curvas não são constantes ao longo do tempo ....................................21

Figura 3. Curvas diferem entre os tratamentos ao longo do tempo ..........................21

Figura 4. Esquema da análise de medidas repetidas no proc GLM do SAS ..............48

Figura 5. Esquema da análise de medidas repetidas no proc MIXED do SAS ...........49

Figura 6. Perfis médios dos tratamentos ao longo dos dias ...................................63

Figura 7. Modelo ajustado para os tratamentos ao longo dos dias .........................68

Figura 8. Dispersão dos valores estimados vs. resíduos .....................................69

Figura 9. Gráfico de probabilidade normal ..................................................... 70

Figura 10. Modelo final ajustado para os tratamentos ao longo dos dias .................71 


\section{LISTA DE TABELAS}

Página

Tabela 1. Análise da Variância, Esperanças dos Quadrados Médios e Teste F.......19

Tabela 2. Dados obtidos do experimento com Bromélias em função dos tratamentos e das medidas realizadas ao longo do tempo para a variável Número Médio de Folhas

Tabela 3. Resumo de comparações de procedimentos para análise de medidas

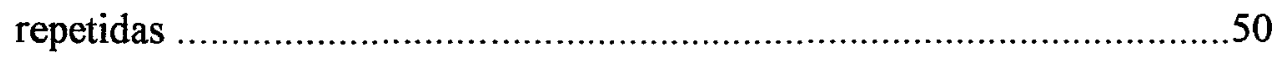

Tabela 4. Resultados da análise univariada usando o proc GLM .........................55

Tabela 5. Testes multivariados para os fatores intra-indivíduos .........................57

Tabela 6. Teste de Esfericidade ...............................................................58

Tabela 7. Testes univariados e correções para o número de graus de liberdade para os efeitos intra-indivíduos .................................................58

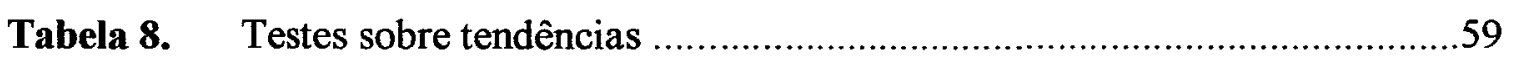

Tabela 9. Informações sobre os modelos univariados com estruturas para a matriz de covariâncias do tipo Huynh-Feldt e Sem Estrutura utilizando o proc MIXED

Tabela 10. Testes para os efeitos fixos dos modelos com estruturas para a matriz de covariâncias do tipo Huynh-Feldt e Sem Estrutura utilizando o proc MIXED

Tabela 11. Testes multivariados produzidos pelo proc MIXED …........................62

Tabela 12. Critério de informação de Akaike (AIC) para o modelo completo .........65

Tabela 13. Testes para os efeitos fixos do modelo completo com a estrutura da matriz de covariâncias do tipo Sem Estrutura ...................................66

Tabela 14. Critério de informação de Akaike (AIC) para o modelo reduzido ... .66 
Tabela 15. Testes para os efeitos fixos do modelo completo com a estrutura da matriz de covariâncias do tipo Sem Estrutura com Correlações

Tabela 16. Teste de Tukey-Kramer para o fator tratamentos do modelo reduzido com a estrutura da matriz de covariâncias do tipo Sem Estrutura com Correlações 68

Tabela 17. Estimativas do modelo final ajustado 70

Tabela 18. Estruturas da matriz de covariâncias utilizadas para as simulaçõe .74

Tabela 19. Valores da estatística $\chi^{2}$ para o teste de aderência da distribuição de freqüência dos níveis mínimos de significância associados aos valores da estatística $F$ para Dias e interação Tratamentos $\times$ Dias, com dados balanceados e desbalanceados para a distribuição normal .75

Tabela 20. Valores da estatística $\chi^{2}$ para o teste de aderência da distribuição de freqüência dos níveis mínimos de significância associados aos valores da estatística $F$ para Dias e interação Tratamentos $\times$ Dias, com dados balanceados e desbalanceados para a distribuição normal contaminada

Tabela 21. Frequências dos níveis mínimos de significância nas primeiras classes, associados aos valores da estatística F para Dias e interação Tratamentos $\times$ Dias, com dados balanceados e desbalanceados para a distribuição normal 79

Tabela 22. Frequências dos níveis mínimos de significância nas primeiras classes, associados aos valores da estatística $F$ para Dias e interação Tratamentos $\times$ Dias, com dados balanceados e desbalanceados para a distribuição normal contaminada $\operatorname{com} \alpha=0,05$ e $(1-\alpha)=0,95$ .80

Tabela 23. Frequências dos níveis mínimos de significância nas primeiras classes, associados aos valores da estatística F para Dias e interação Tratamentos $\times$ Dias, com dados balanceados e desbalanceados para a distribuição normal contaminada $\operatorname{com} \alpha=0,10$ e $(1-\alpha)=0,90$ 80 


\title{
MODELOS UNIVARIADO E MULTIVARIADO PARA ANÁLISE DE MEDIDAS REPETIDAS E VERIFICAÇÃO DA ACURÁCIA DO MODELO UNIVARIADO POR MEIO DE SIMULAÇÃO
}

\author{
Autor: Lara Hoffmann Xavier \\ Orientador: Prof. Dr. Carlos Tadeu dos Santos Dias
}

\section{RESUMO}

O presente trabalho discute algumas técnicas para análise de dados de medidas repetidas, utilizando modelos univariado, multivariado e misto.

Discutem-se também algumas questões sobre o problema, bem como alguns procedimentos para seleção do melhor modelo, quando a abordagem de estimação dos parâmetros do modelo misto é via máxima verossimilhança e máxima verossimilhança restrita.

Quanto ao modelo univariado de medidas repetidas, foram realizadas simulações para verificação da acurácia dos testes quando as suposições exigidas são válidas, ou não.

Este trabalho também discute e compara os procedimentos GLM e MIXED do SAS ${ }^{\circledR}$, quando utilizados para análise de dados de medidas repetidas. 


\title{
UNIVARIATE AND MULTIVARIATE MODELS FOR THE ANALYSIS OF REPEATED MEASURES AND ACCURACY VERIFICATION OF THE UNIVARIATE MODEL THROUGH SIMULATION
}

\author{
Author: Lara Hoffmann Xavier \\ Adviser: Prof. Dr. Carlos Tadeu dos Santos Dias
}

\section{SUMMARY}

Some technical aspects of repeated measures analysis, using univariate, multivariate and mixed models are discussed in this dissertation.

Some problems and procedures to select the adequate model, when the parameters estimation are effectuated through maximum likelihood and restricted maximum likelihood estimation.

As for repeated measures, univariate models simulations were done to verify the accuracy of the tests, both when the assumptions are valid or not.

This dissertation also discusses and compares the SAS procedures GLM and MIXED when utilized for repeated measures analysis. 


\section{INTRODUÇÃO}

Experimentos com medidas repetidas no tempo são bastante comuns na prática, sendo utilizados por pesquisadores de diversas áreas, quando o objetivo é verificar o comportamento de um determinado indivíduo, ao longo do tempo, seja o mesmo uma planta, um animal, uma máquina, uma pessoa, uma empresa etc.

Neste trabalho será enfocado o caso em que as avaliações de uma determinada característica, em vários tempos, serão realizadas sobre o mesmo indivíduo, produzindo uma determinada forma de relação entre as observações obtidas. E, por esse motivo, experimentos assim realizados, requerem outras suposições, além das usuais, para que a análise seja correta e os testes produzam resultados válidos.

Dados de medidas repetidas no tempo, tanto podem ser analisados através de um modelo univariado, que impõe uma restrição rigorosa para a matriz de covariâncias, como por meio de um modelo multivariado, que adota uma matriz de covariâncias sem restrições, ou seja, a matriz é sem estrutura, ou ainda através de um modelo misto, que possibilita a utilização de diferentes estruturas para a matriz de covariâncias.

Essas técnicas serão empregadas utilizando-se um delineamento aleatorizado em blocos.

Os objetivos deste trabalho são:

a) discutir algumas técnicas utilizadas para a análise de dados de medidas repetidas, através de modelos univariado, multivariado e misto; 
b) no caso do modelo univariado, que é o mais utilizado na prática, simular algumas situações em que as suposições do modelo são válidas, ou não, para verificar a eficiência dos testes, $\mathrm{e}$

c) realizar comparações entre os procedimentos GLM e MIXED do SAS quando se tem interesse em trabalhar com dados de medidas repetidas. 


\section{REVISÃO DE LITERATURA}

O termo medidas repetidas, segundo Diggle (1988) e Crowder \& Hand (1990), é usado para designar medidas feitas na mesma variável ou na mesma unidade experimental em mais de uma ocasião.

A estrutura de parcelas subdivididas, em um estudo de medidas repetidas no tempo, é caracterizada quando se aplicam às parcelas os níveis do fator $\mathrm{A}$ e nos quais se tomam medidas repetidas, em ocasiões sucessivas, sob a mesma parcela, admitindo-se que essas medidas, tomadas em ocasiões distintas, têm variâncias homogêneas e são igualmente correlacionadas.

Em estudos de medidas repetidas no tempo, em um delineamento no esquema de parcelas subdivididas, por exemplo, os níveis desse tempo não podem ser aleatorizados para seus intervalos. Dessa forma, a análise de variância usual pode não ser válida, porque com a falta de aleatorização os erros correspondentes às respectivas unidades experimentais ou indivíduos podem ter uma matriz de covariânciạs que não é igual àquela exigida para que a análise usual de um delineamento seja válida, isto é, variâncias homogêneas.

Fernandez (1991) também salienta o problema de que quando o experimento é sistematicamente arranjado, sem aleatorização, a análise de um experimento de medidas repetidas com delineamento de parcelas subdivididas pode inflacionar a probabilidade de falsamente rejeitar a hipótese nula (erro tipo I).

Para o modelo de análise de parcelas subdivididas, são feitas pressuposições de que, tanto o erro da parcela, que engloba o fator de tratamentos ou grupos, como o erro da subparcela, onde são alocados os tempos e a interação 
tempos $\times$ tratamentos, tenham distribuição normal, sejam independentes e identicamente distribuídos, com variâncias constantes, cujas pressuposições são as mesmas feitas para uma análise usual. $\mathrm{O}$ erro da parcela também é conhecido como erro entre indivíduos, e o erro da subparcela como intra-individuos.

Huynh \& Feldt (1970) mostraram que, em um delineamento de parcelas subdivididas com medidas repetidas no tempo, o teste $F$ com relação à parcela tem distribuição $\mathrm{F}$ exata, mas com relação à subparcela, só terá distribuição $\mathrm{F}$ exata se a matriz de covariâncias satisfizer certa pressuposição, além das citadas anteriormente.

De acordo com Milliken \& Johnson (1992), tais pressuposições nem sempre são apropriadas para um delineamento de parcelas subdivididas com medidas repetidas no tempo, sendo, porém, uma análise correta quando realizada sob suposições mais gerais. Essas suposições mais gerais requerem certa forma para a matriz de covariâncias dos erros denotada por $\Sigma$.

Uma condição suficiente para que o teste $F$ da análise de variância usual, em nível de subparcela, para o fator tempos e interação tempos $\times$ tratamentos, seja válido, é que a matriz de covariâncias tenha uma forma chamada de simetria composta, que ocorre quando a matriz de covariâncias $\Sigma$ puder ser expressa, por exemplo, como:

$$
\Sigma=\left[\begin{array}{cccc}
\left(\sigma^{2}+\sigma_{1}^{2}\right) & \sigma_{1}^{2} & \sigma_{1}^{2} & \sigma_{1}^{2} \\
\sigma_{1}^{2} & \left(\sigma^{2}+\sigma_{1}^{2}\right) & \sigma_{1}^{2} & \sigma_{1}^{2} \\
\sigma_{1}^{2} & \sigma_{1}^{2} & \left(\sigma^{2}+\sigma_{1}^{2}\right) & \sigma_{1}^{2} \\
\sigma_{1}^{2} & \sigma_{1}^{2} & \sigma_{1}^{2} & \left(\sigma^{2}+\sigma_{1}^{2}\right)
\end{array}\right]
$$

onde:

$\sigma^{2}$ : é a variância da subparcela (intra-individuos);

$\sigma_{1}^{2}$ : é a variância da parcela (entre indivíduos).

A condição de simetria composta implica que a variável aleatória seja igualmente correlacionada e tenha variâncias iguais, considerando as diferentes ocasiões. 
Uma condição mais geral da forma de $\Sigma$ é descrita por Huynh \& Feldt (1970). Essa condição, denominada de HUYNH-FELDT (H-F), especifica que os elementos da matriz de covariâncias $\Sigma$ sejam expressos, para um $\lambda>0$, como

$$
\Sigma=\left[\begin{array}{cccc}
\sigma_{1}^{2} & \frac{\left(\sigma_{1}^{2}+\sigma_{2}^{2}\right)}{2}-\lambda & \frac{\left(\sigma_{1}^{2}+\sigma_{3}^{2}\right)}{2}-\lambda & \frac{\left(\sigma_{1}^{2}+\sigma_{4}^{2}\right)}{2}-\lambda \\
\frac{\left(\sigma_{2}^{2}+\sigma_{1}^{2}\right)}{2}-\lambda & \sigma_{2}^{2} & \frac{\left(\sigma_{2}^{2}+\sigma_{3}^{2}\right)}{2}-\lambda & \frac{\left(\sigma_{2}^{2}+\sigma_{4}^{2}\right)}{2}-\lambda \\
\frac{\left(\sigma_{3}^{2}+\sigma_{1}^{2}\right)}{2}-\lambda & \frac{\left(\sigma_{3}^{2}+\sigma_{2}^{2}\right)}{2}-\lambda & \sigma_{3}^{2} & \frac{\left(\sigma_{3}^{2}+\sigma_{4}^{2}\right)}{2}-\lambda \\
\frac{\left(\sigma_{4}^{2}+\sigma_{1}^{2}\right)}{2}-\lambda & \frac{\left(\sigma_{4}^{2}+\sigma_{2}^{2}\right)}{2}-\lambda & \frac{\left(\sigma_{4}^{2}+\sigma_{3}^{2}\right)}{2}-\lambda & \sigma_{4}^{2}
\end{array}\right],
$$

onde $\lambda$ é a diferença entre a média das variâncias e a média das covariâncias.

Por exemplo, se a matriz de covariâncias tivesse a seguinte forma:

$$
\Sigma=\left[\begin{array}{cccc}
5,0 & 2,5 & 5,0 & 7,5 \\
2,5 & 10,0 & 7,5 & 10,0 \\
5,0 & 7,5 & 15,0 & 12,5 \\
7,5 & 10,0 & 12,5 & 20,0
\end{array}\right]
$$

$\lambda$ seria calculado da seguinte maneira:

$$
\begin{gathered}
\lambda=\text { média das variâncias }- \text { média das covariâncias } \\
\lambda=\frac{(5,0+10,0+15,0+20,0)}{4}-\frac{(2,5+5,0+7,5+7,5+10,0+12,5)}{6}= \\
\lambda=12,5-7,5=5
\end{gathered}
$$

Assim, por exemplo, o elemento $\mathrm{a}_{12}$ da matriz é obtido por:

$$
a_{12}=\frac{\left(\sigma_{1}^{2}+\sigma_{2}^{2}\right)}{2}-\lambda=\frac{(5,0+10,0)}{2}-5,0=7,5-5,0=2,5
$$

A condição de H-F é uma condição necessária e suficiente para que o teste F da análise de variância usual, no esquema de delineamento de parcelas subdivididas no tempo, seja válido. A condição de H-F é equivalente a especificar que variâncias da diferença entre pares de erros sejam todas iguais, e se as variâncias são todas iguais então a condição é equivalente à de simetria composta. 
Isso pode ser verificado utilizando-se novamente a matriz (1), calculandose todas as variâncias das diferenças dos possíveis pares de erros:

$$
\sigma_{Y_{j}-Y_{j^{\prime}}}^{2}=\sigma_{j}^{2}+\sigma_{j^{\prime}}^{2}-2 \sigma_{i j^{\prime}}=a,
$$

constante para todo $\mathrm{j}$ e $\mathrm{j}^{\prime}\left(\mathrm{j} \neq \mathrm{j}^{\prime}\right)$

$$
\begin{aligned}
& \sigma_{\mathrm{Y}_{1}-\mathrm{Y}_{2}}^{2}=5,0+10,0-2(2,5)=10,0 \\
& \sigma_{\mathrm{Y}_{1}-\mathrm{Y}_{3}}^{2}=5,0+15,0-2(5,0)=10,0 \\
& \sigma_{\mathrm{Y}_{1}-\mathrm{Y}_{4}}^{2}=5,0+20,0-2(7,5)=10,0 \\
& \sigma_{\mathrm{Y}_{2}-\mathrm{Y}_{3}}^{2}=10,0+15,0-2(7,5)=10,0 \\
& \sigma_{\mathrm{Y}_{2}-\mathrm{Y}_{4}}^{2}=10,0+20,0-2(10,0)=10,0 \\
& \sigma_{\mathrm{Y}_{3}-\mathrm{Y}_{4}}^{2}=15,0+20,0-2(12,5)=10,0
\end{aligned}
$$

Desde que $\sigma_{\mathrm{Y}_{\mathrm{j}} \mathrm{Y}_{\mathrm{j}}}^{2}$ seja igual a uma constante para todo $\mathrm{j}$ e j' $(\mathrm{j} \neq \mathrm{j}$ '), $\Sigma$ é dita do tipo H-F.

As matrizes de covariâncias $\Sigma$, na forma da simetria composta e erros independentes, são casos especiais da condição de H-F, isto é, a covariância é a média das variâncias.

Um problema com relação à validade dos testes surge quando se têm estruturas da matriz de covariâncias diferentes das estruturas de simetria composta, erros independentes e da condição de H-F, levando a testes $\mathrm{F}$ não exatos.

Para se verificar se a matriz de covariâncias atende à condição de H-F, Mauchly (1940) propôs um teste chamado teste de esfericidade, que verifica se uma população multivariada apresenta variâncias iguais e correlações nulas.

Meredith \& Stehman (1991) verificaram que a violação da condição de HF leva a testes muito liberais para os fatores da subparcela, para tempos e para a interação tempos $\times$ tratamentos.

Quando a estrutura envolvida apresenta outra forma é necessário utilizar outros métodos para encontrar um modelo que permita a utilização da estrutura da matriz de covariâncias que melhor represente o conjunto de dados em questão, ou então a 
utilização de um fator de correção para o número de graus de liberdade do fator da subparcela.

A seleção do melhor modelo de estrutura da matriz $\Sigma$ pode, então, ser feita utilizando o Critério de Informação de Akaike (AIC) ou através de um teste de razão de máxima verossimilhança.

\subsection{Teste de Esfericidade de Mauchly}

Mauchly (1940) apresenta a estatística de teste para a condição de esfericidade, que verifica se uma população normal multivariada apresenta variâncias iguais e as correlações nulas. Caso uma população apresente essa simetria, será chamada de "esférica".

Esse teste utiliza a condição de H-F para a matriz de covariâncias das $\mathbf{t}$ medidas repetidas dos indivíduos requeridos nos ( $\mathrm{t}-1)$ contrastes ortogonais normalizados, para as medidas repetidas não correlacionadas com variâncias iguais.

Pode-se dizer que dois contrastes são ortogonais quando a soma dos pares de produtos dos coeficientes dos contrastes forem iguais a zero (contrastes perpendiculares).

A ortogonalidade dos contrastes garante que:

a) cada contraste é associado a uma única porção da variabilidade explicada pelo efeito que se está testando;

b) está sendo testado o número máximo de hipóteses, onde cada hipótese é associada a uma única porção da variabilidade explicada pelo modelo;

c) o teste é aproximadamente independente.

Para $\mathrm{t}$ tempos existem mais de um conjunto de (t-1) contrastes ortogonais, sendo que um contraste ortogonal será normalizado quando for dividido pela sua norma euclidiana. 
Seja a matriz $\Sigma$ de covariâncias das medidas repetidas. A condição que $H-$ F requer para as covariâncias dos contrastes é

$$
\mathrm{C}_{(\mathrm{t}-1) \times \mathrm{t}} \sum_{(\mathrm{(xt})} \mathrm{C}^{\prime}{ }_{\mathrm{t} \times(\mathrm{t}-1)}=\lambda \mathrm{I}_{(\mathrm{t}-1) \times(\mathrm{t}-1)}
$$

onde:

C: é a matriz dos coeficientes dos contrastes ortogonais normalizados que representa o total de hipóteses nulas;

$\Sigma$ : é a matriz de covariâncias;

$\lambda$ : é um escalar maior do que zero e

I: é a matriz identidade.

Se a condição (2) for satisfeita, a matriz de covariâncias $\Sigma$ será dita esférica.

Kuehl (1994) e Kirk (1995) descrevem o teste de esfericidade da seguinte forma: seja $\mathrm{s}_{\mathrm{ij}} \mathrm{o}$ elemento na i-ésima linha e j-ésima coluna da matriz de covariâncias amostral $\mathrm{S}_{(\mathrm{t} x \mathrm{t})}$, para o erro intra-indivíduos, com $v$ graus de liberdade. Escolhem-se (t-1) contrastes ortogonais normalizados nas $t$ medidas repetidas, e sendo a matriz $\mathrm{C}_{(t-1) \times t}$, onde as linhas são contrastes ortogonais normalizados nas $\mathrm{t}$ medidas repetidas, calcula-se a matriz $\operatorname{CSC}_{(t-1) \times(-1))}^{\prime}$. Então, a estatística de teste formulada por Mauchly (1940) para a hipótese nula

será

$$
\mathrm{H}_{0}: \mathrm{C}^{\mathrm{C}} \mathrm{C}^{\prime}=\lambda \mathrm{I}
$$

$$
\mathrm{W}=\frac{(\mathrm{t}-1)^{t-1}\left|\operatorname{CSC}^{\prime}\right|}{\left(\operatorname{tr}\left(\operatorname{CSC}^{\prime}\right)\right)^{t-1}}
$$

com

$$
\mathrm{f}=\frac{1}{2} \mathrm{t}(\mathrm{t}-1)-1 \text { graus de liberdade }
$$

utilizando-se ainda, para melhorar a acurácia desta aproximação pela distribuição de Quiquadrado, um fator de escala definido como 


$$
\gamma=(\mathrm{gb}-\mathrm{g}-\mathrm{b}+1)-\frac{2 \mathrm{t}^{2}-3 \mathrm{t}+3}{6(\mathrm{t}-1)}
$$

A hipótese nula será rejeitada ao nível $\alpha$ de significância se

$$
-\gamma \ln \mathrm{W}>\chi_{\alpha, \mathrm{f}}^{2} \text {. }
$$

Koch et al. (1985), Von Ende (1993) comentam e Vonesh \& Chinchilli (1997) mostram que o teste de esfericidade não é muito poderoso para amostras pequenas e não é robusto quando há violação da suposição de normalidade.

Segundo Milliken (1984), Vonesh \& Chinchilli (1997), a análise de medidas repetidas tornar-se-á mais complexa quando houver mais de um fator em estudo com medidas repetidas, por exemplo, se existirem dois fatores intra-indivíduos como tempo, dias e a interação tempoxdias. Nesse caso haveria necessidade de se construir um teste de esfericidade, um teste $\mathrm{F}$ e correções para os números de graus de liberdade para cada um desses fatores intra-individuos.

No caso das pressuposições de normalidade, de independência e da condição de H-F para a matriz $\Sigma$ de covariâncias não serem satisfeitas, uma alternativa seria a análise multivariada, também conhecida como análise de perfis, que adota uma hipótese mais geral sobre a estrutura da matriz de covariâncias. Outra possibilidade seria utilizar análise univariada no esquema de delineamento de parcelas subdivididas no tempo, realizando o ajuste do número de graus de liberdade do teste $\mathrm{F}$ para o fator da subparcela.

As correções para os números de graus de liberdade foram inicialmente propostas por Box (1945 a, b), e aperfeiçoadas por Geisser \& Greenhouse (1958) e Huynh \& Feldt (1976). Essas correções são efetuadas pela multiplicação de um valor pelo número de graus de liberdade do fator da subparcela. Caso os dados sejam representados por uma matriz de covariâncias que não se adeque às duas técnicas citadas anteriormente, uma opção seria o ajuste de modelos mistos que podem envolver curvas de crescimento ou modelos polinomiais, que incluam a matriz de covariâncias que melhor explique o comportamento dos dados. Esses modelos são construídos levando-se em 
conta vários tipos de estruturas da matriz de covariâncias, sendo que o melhor modelo poderia ser escolhido por um teste de razão de verossimilhança ou pelo critério de informação de Akaike que penaliza os modelos com um número grande de parâmetros.

Fernandez (1991) sugere que: a) se a condição de H-F para a matriz de covariâncias for satisfeita (teste de esfericidade não significativo) o teste univariado pode ser utilizado; b) se a condição H-F para a matriz de covariâncias não for satisfeita, e o nível de significância do teste de esfericidade estiver entre 0,05 e 0,01 , poderão ser utilizados a correção para os números de graus de liberdade ou os testes multivariados, e c) se a condição de H-F para a matriz de covariâncias for rejeitada, com um nível de significância menor que 0,01 , somente testes multivariados deverão ser utilizados. Aqui o autor não faz nenhuma referência à escolha de outro modelo que não seja o univariado ou multivariado.

Diggle (1988) comenta sobre algumas dificuldades que surgem na análise de medidas repetidas, tais como: a) a resposta para cada indivíduo ser uma seqüência de medidas em uma escala contínua; b) a resposta média depender tanto do tratamento quanto do tempo em que a medida foi realizada e c) fazer inferências sobre os efeitos dos tratamentos em um perfil médio de resposta. Sugere a utilização de semivariogramas para a seleção inicial das matrizes de covariâncias, podendo incorporar uma estrutura de correlação serial no modelo de medidas repetidas. Outros detalhes sobre esse enfoque são fornecidos por Diggle et al. (1998).

\subsection{Modelo Multivariado}

Como alternativa para a análise de medidas repetidas no tempo, tem-se a análise multivariada, que pode apresentar menor poder em seus testes e às vezes indicar diferenças significativas onde realmente não existem. Mas, esses riscos podem ser minimizados garantindo-se que os erros tenham distribuição normal multivariada. Por 
esse motivo, de acordo Singer \& Andrade (1986) a análise de variância multivariada, também conhecida como análise multivariada de perfis, é uma solução natural para dados de medidas repetidas.

Meredith \& Stehman (1991) também salientam que não há suposição sobre a estrutura da matriz de covariâncias, sendo, por isso, uma solução natural para dados de medidas repetidas.

Segundo Vonesh \& Chinchilli (1997), geralmente, as técnicas usuais impõem suposições de que todas observações sejam independentes, mas essa suposição não é adequada para dados de medidas repetidas onde as observações feitas no mesmo indivíduo usualmente são correlacionadas.

Vonesh \& Chinchilli (1997) sugerem, para um experimento com delineamento de parcelas subdivididas, com medidas repetidas no tempo, o seguinte modelo:

$$
\mathrm{y}_{\mathrm{ijk}}=\mu+\beta_{\mathrm{i}}+\tau_{\mathrm{j}}+\gamma_{\mathrm{k}}+(\tau \gamma)_{\mathrm{jk}}+\mathrm{e}_{\mathrm{ijk}}
$$

onde:

$\mathrm{y}_{\mathrm{ijk}}$ : é o valor observado para a variável resposta no k-ésimo tempo para o j-ésimo tratamento no i-ésimo bloco;

$\mu$ : é uma constante inerente a todas as observações;

$\beta_{\mathrm{i}}$ : $\quad$ é o efeito do i-ésimo bloco;

$\tau_{\mathrm{j}}$ : é o efeito do j-ésimo tratamento;

$\gamma_{\mathrm{k}}$ : é o efeito do k-ésimo tempo observado;

$(\tau \gamma)_{\mathbf{j k}}$ : é o efeito da interação entre o j-ésimo tratamento com o k-ésimo tempo;

$\mathrm{e}_{\mathrm{ijk}}$ : é o erro aleatório correspondente às observações do k-ésimo tempo para o iésimo bloco no j-ésimo tratamento (variação do acaso sobre as observações), supostos homocedásticos, independentes e normalmente distribuídos.

onde: 
$\mathrm{i}=1, \ldots, \mathrm{b}$ é o índice para níveis do fator blocos;

$\mathrm{j}=1, \ldots, \mathrm{g}$ é o índice para níveis do fator entre indivíduos (tratamentos);

$\mathrm{k}=1, \ldots, \mathrm{t}$ é $\mathrm{o}$ índice para níveis do fator intra-indivíduos (tempos).

Observe-se que nesse modelo (3), o erro da parcela não é incluído.

Para que esse modelo tenha posto completo, é necessário impor as seguintes restrições:

$\sum_{\mathrm{i}=1}^{\mathrm{b}} \beta_{\mathrm{i}}=0 ; \quad \sum_{\mathrm{j}=1}^{\mathrm{g}} \tau_{\mathrm{j}}=0 ; \quad \sum_{\mathrm{k}=1}^{\mathrm{t}} \gamma_{\mathrm{k}}=0 ; \quad \sum_{\mathrm{j}=1}^{\mathrm{g}}(\tau \gamma)_{\mathrm{jk}}=0$, para $\mathrm{k}=1, \ldots, \mathrm{t} \quad \mathrm{e} \sum_{\mathrm{k}=1}^{\mathrm{t}}(\tau \gamma)_{\mathrm{jk}}=0$, para $\mathbf{j}=1, \ldots, \mathrm{g}$ e com vetor aleatório

$$
\mathrm{e}_{\mathrm{ijk}}^{\prime}=\left[\begin{array}{lll}
\mathrm{e}_{\mathrm{ij} 1} & \cdots & \mathrm{e}_{\mathrm{ijt}}
\end{array}\right] \text { กi.i.d. } \mathrm{N}_{\mathrm{t}}(0, \Sigma)
$$

onde $\Sigma$ é uma matriz $\mathrm{t} \times \mathrm{t}$, positiva definida com uma estrutura geral.

Nesse caso, utilizando-se a análise multivariada, as hipóteses de interesse testadas são:

a) a hipótese de igualdade do efeito de tratamentos, que corresponde à hipótese de perfis coincidentes;

b) a hipótese de igualdade do efeito de tempos, que corresponde à hipótese de perfis constantes;

c) a hipótese de não interação de tempos $\times$ tratamentos, que corresponde à hipótese de perfis paralelos.

Assim a matriz de covariâncias $\Sigma$ desse modelo é sem estrutura com $\mathrm{t}(\mathrm{t}+1) / 2$ parâmetros.

A análise multivariada de perfis também pode ser feita através de um modelo matricial expresso da seguinte maneira:

$$
\mathrm{Y}=\mathrm{XB}+\Psi
$$

onde:

$\mathrm{Y}$ : é a matriz dos dados observados gb $\mathrm{gt}$ de $\mathrm{t}$ respostas para os $\mathrm{n}$ indivíduos; 
$\mathrm{X}$ : é a matriz $\mathrm{gb} \times(\mathrm{g}+\mathrm{b}+1)$ de delineamento conhecida. Essa matriz corresponde aos valores da variável explanatória e das variáveis "dummy" associadas com a classificação das variáveis;

B: é a matriz $(g+b+1) \times t$ de parâmetros dos efeitos fixos desconhecidos;

$\Psi$ : é a matriz $g b \times t$ do erro experimental.

Esse modelo assume que $\Psi$ de (4) seja uma matriz de variáveis aleatórias independentes, onde as linhas são não correlacionadas e têm uma distribuição normal multivariada com a média 0 e matriz de covariâncias $\Sigma$. Assim, para o modelo usual linear multivariado, assumindo

$$
\mathrm{E}\left[\mathrm{e}_{\mathrm{i}}\right]=0 \text { e } \operatorname{Var}\left[\mathrm{e}_{\mathrm{i}}\right]=\Sigma, \mathrm{i}=1,2, \ldots, \mathrm{n}
$$

onde $\Psi_{i}$ de (4) é o vetor de erro experimental associado com o i-ésimo indivíduo, na forma matricial tem-se que

$$
\begin{aligned}
& \mathrm{Y}_{(\mathrm{gb} \times \mathrm{t})}=\left[\begin{array}{cccc}
\mathrm{y}_{111} & \mathrm{y}_{112} & \cdots & \mathrm{y}_{11 \mathrm{t}} \\
\mathrm{y}_{121} & \mathrm{y}_{122} & \cdots & \mathrm{y}_{12 \mathrm{t}} \\
\vdots & \vdots & & \vdots \\
\mathrm{y}_{1 b 1} & \mathrm{y}_{1 b 2} & \cdots & \mathrm{y}_{1 \mathrm{bt}} \\
\vdots & \vdots & & \vdots \\
\mathrm{y}_{\mathrm{g} 11} & \mathrm{y}_{\mathrm{g} 12} & \cdots & \mathrm{y}_{\mathrm{glt}} \\
\vdots & \vdots & & \vdots \\
\mathrm{y}_{\mathrm{gbl}} & \mathrm{y}_{\mathrm{gb2}} & \cdots & \mathrm{y}_{\mathrm{gbt}}
\end{array}\right] \quad \Psi_{(\mathrm{gb} \times \mathrm{t})}=\left[\begin{array}{cccc}
\mathrm{e}_{111} & \mathrm{e}_{112} & \cdots & \mathrm{e}_{11 \mathrm{t}} \\
\mathrm{e}_{121} & \mathrm{e}_{122} & \cdots & \mathrm{e}_{12 \mathrm{t}} \\
\vdots & \vdots & & \vdots \\
\mathrm{e}_{1 b 1} & \mathrm{e}_{1 \mathrm{~b} 2} & \cdots & \mathrm{e}_{1 \mathrm{bt}} \\
\vdots & \vdots & & \vdots \\
\mathrm{e}_{\mathrm{g} 11} & \mathrm{e}_{\mathrm{g} 12} & \cdots & \mathrm{e}_{\mathrm{glt}} \\
\vdots & \vdots & & \vdots \\
\mathrm{e}_{\mathrm{gbl}} & \mathrm{e}_{\mathrm{gb} 2} & \cdots & \mathrm{e}_{\mathrm{gbt}}
\end{array}\right] \\
& X_{(g b \times(g+b+1))}=\left[X_{1} \vdots X_{2} \vdots X_{3}\right] \text {, }
\end{aligned}
$$

$\operatorname{com} X_{1}=\left\lfloor l_{g b}\right\rfloor, X_{2}=\left[\begin{array}{l}I_{b} \\ I_{b} \\ \cdots \\ I_{b}\end{array}\right]$ e $X_{3}=\left[\begin{array}{cccc}1_{b} & 0 & \cdots & 0 \\ 0 & 1_{b} & \cdots & 0 \\ \vdots & \vdots & & \vdots \\ 0 & 0 & \cdots & 1_{b}\end{array}\right]$,

onde $1_{(\mathrm{g} \times \mathrm{x})}$ é um vetor de uns, $\mathrm{I}_{(\mathrm{b} \times \mathrm{b})}$ é uma matriz identidade e $1_{(\mathrm{b} \times 1)}$ é um vetor de uns, e 


$$
\mathrm{B}_{((\mathrm{g}+\mathrm{b}+\mathrm{l}) \times \mathrm{t})}=\left[\begin{array}{cccc}
\mu_{1} & \mu_{2} & \cdots & \mu_{\mathrm{t}} \\
\beta_{11} & \beta_{12} & \cdots & \beta_{1 \mathrm{t}} \\
\beta_{21} & \beta_{22} & \cdots & \beta_{2 \mathrm{t}} \\
\vdots & \vdots & & \vdots \\
\beta_{\mathrm{b} 1} & \beta_{\mathrm{b} 2} & \cdots & \beta_{\mathrm{bt}} \\
\tau_{11} & \tau_{12} & \cdots & \tau_{1 \mathrm{t}} \\
\tau_{21} & \tau_{22} & \cdots & \tau_{2 \mathrm{t}} \\
\vdots & \vdots & & \vdots \\
\tau_{\mathrm{g} 1} & \tau_{\mathrm{g} 2} & \cdots & \tau_{\mathrm{gt}}
\end{array}\right]
$$

Supõe-se que os perfis de respostas $Y_{\mathrm{ijk}}$ obedeçam às distribuições Normais t-variadas e que as matrizes de covariâncias correspondentes sejam todas iguais e sigam a forma geral:

$$
\Sigma=\left[\begin{array}{cccc}
\sigma_{1}^{2} & \sigma_{12} & \ldots & \sigma_{1 \mathrm{t}} \\
\sigma_{21} & \sigma_{2}^{2} & \ldots & \sigma_{2 \mathrm{t}} \\
\vdots & \vdots & & \vdots \\
\sigma_{\mathrm{t} 1} & \sigma_{\mathrm{t} 2} & \ldots & \sigma_{\mathrm{t}}^{2}
\end{array}\right]
$$

Aqui, as hipóteses de interesse são:

a) $\mathrm{H}_{01}$ : os perfis médios de resposta correspondentes são paralelos, isto é, não existe interação entre o fator tratamentos e o fator tempos. $\mathrm{Na}$ forma matricial, em relação aos parâmetros do modelo:

$$
\mathrm{H}_{0 \mathrm{I}}:\left[\begin{array}{c}
\mu_{111}-\mu_{112} \\
\mu_{112}-\mu_{113} \\
\vdots \\
\mu_{1 \mathrm{~b}(\mathrm{t}-1)}-\mu_{\mathrm{lbt}}
\end{array}\right]=\cdots=\left[\begin{array}{c}
\mu_{\mathrm{g} 11}-\mu_{\mathrm{g} 12} \\
\mu_{\mathrm{g} 12}-\mu_{\mathrm{g} 13} \\
\vdots \\
\mu_{\mathrm{gb}(\mathrm{t}-1)}-\mu_{\mathrm{gbt}}
\end{array}\right]
$$


b) $\mathrm{H}_{0 \mathrm{G}}$ : os perfis médios de resposta correspondentes aos diversos tratamentos são coincidentes, isto é, não existe efeito desse fator. $\mathrm{Na}$ forma matricial, em relação aos parâmetros do modelo:

$$
\mathrm{H}_{0 \mathrm{G}}:\left[\begin{array}{c}
\mu_{111} \\
\mu_{112} \\
\vdots \\
\mu_{1 \mathrm{bt}}
\end{array}\right]=\cdots=\left[\begin{array}{c}
\mu_{\mathrm{g} 11} \\
\mu_{\mathrm{g} 12} \\
\vdots \\
\mu_{\mathrm{gbt}}
\end{array}\right]
$$

c) $\mathrm{H}_{0 \mathrm{~T}}$ : os perfis médios de resposta correspondentes aos diversos tratamentos são paralelos ao eixo das abcissas, isto é, não existe efeito do fator tempos, em relação aos parâmetros do modelo:

$$
\mathrm{H}_{0 \mathrm{~T}}:\left[\begin{array}{c}
\mu_{111} \\
\mu_{211} \\
\vdots \\
\mu_{\mathrm{gbl}}
\end{array}\right]=\cdots=\left[\begin{array}{c}
\mu_{11 \mathrm{t}} \\
\mu_{21 \mathrm{t}} \\
\vdots \\
\mu_{\mathrm{gbt}}
\end{array}\right]
$$

As hipóteses a serem testadas também podem ser expressas na forma da hipótese linear geral:

$$
\mathrm{H}: \mathrm{GBT}=0
$$

onde $G_{(g-1) \times(g+b+1)}$ e $T_{t \times(t-l)}$ são matrizes de constantes conhecidas com postos $g \mathrm{e}$ $t$, respectivamente. Tem-se que a matriz $G$ é responsável por comparações entre os grupos (linhas da matriz B), e a matriz $\mathrm{T}$ é responsável por comparações entre os tempos (colunas da matriz B). As seguintes possíveis correspondências podem ser obtidas através da hipótese linear geral (que não são únicas na forma de expressá-las):

$$
\mathrm{H}_{\mathrm{OI}}: \mathrm{G}_{1_{(\mathrm{g}-1) \times(\mathrm{g}+\mathrm{b}+1)}}=\left[\begin{array}{rrrrrrrrr}
0 & 0 & \cdots & 0 & 1 & -1 & 0 & \cdots & 0 \\
0 & 0 & \cdots & 0 & 1 & 0 & -1 & \cdots & 0 \\
\vdots & \vdots & & \vdots & \vdots & \vdots & \vdots & & \vdots \\
0 & 0 & \cdots & 0 & 1 & 0 & 0 & \cdots & -1
\end{array}\right]
$$

e 


$$
\begin{gathered}
\mathrm{T}_{\mathbf{1}_{(\mathrm{t} \times(\mathrm{t}-1))}}=\left[\begin{array}{rrrr}
1 & 0 & \cdots & 0 \\
-1 & 1 & \cdots & 0 \\
0 & -1 & \cdots & 0 \\
\vdots & \vdots & & \vdots \\
0 & 0 & \cdots & 1 \\
0 & 0 & \cdots & -1
\end{array}\right] \\
\mathrm{H}_{\mathrm{OG}}: \mathrm{G}_{1} \text { e } \mathrm{T}_{2}=1_{\mathrm{t}} \\
\mathrm{H}_{\mathrm{OT}}: \mathrm{G}_{2}=1_{(\mathrm{g}+\mathrm{b}+1)}^{\prime} \text { e } \mathrm{T}_{1}
\end{gathered}
$$

onde $1_{t}$ e $1^{\prime}{ }_{(g+b+1)}$ são vetores de uns de dimensões $t$ e $(g+b+1)$ respectivamente e $G_{1}$ e $T_{1}$ definidos como antes.

Andrade \& Singer $(1994)^{1}$, citado por Lima (1996), mostra uma estratégia apresentada por, para a análise de perfis para a situação de não existência de interação entre blocos e tempo, que depende do tipo da matriz $\mathrm{T}$ que define as hipóteses de interesse. Considerando-se três casos: a) todas as colunas de T são contrastes; b) a matriz $\mathrm{T}$ tem uma única coluna que não é um contraste, e c) a matriz $\mathrm{T}$ tem mais de uma coluna e nem todas são contrastes

Segundo Singer \& Andrade (1986) os testes para a hipótese linear geral podem ser obtidos através de diversos critérios. Em geral, as estatísticas de testes correspondentes são funções das raízes características da matriz $\mathrm{HE}^{-1}$, onde

$$
H=T^{\prime}\left(\left(X^{\prime} X\right)^{-1} X^{\prime} Y\right) G^{\prime}\left[G\left(X^{\prime} X\right)^{-1} G^{\prime}\right]^{-1} G\left(\left(X^{\prime} X\right)^{-1} X^{\prime} Y\right) T
$$

é a matriz de soma de quadrados e produtos cruzados devido à hipótese nula, e

$$
E=T^{\prime} Y^{\prime}\left[I-X\left(X^{\prime} X\right)^{-1} X^{\prime}\right]^{-1} Y T
$$

é a matriz de somas de quadrados e produtos cruzados devida ao erro.

\footnotetext{
${ }^{1}$ ANDRADE, D.F.; SINGER, J.M. Profile analysis for randomized complete block experiments. Relatório Técnico No.6772. IME - USP. 1994
} 
Várias estatísticas de testes disponíveis são obtidas através dos princípios da união-intersecção de Roy e da razão de verossimilhança de Wilks. Definindo $\theta_{\mathrm{i}}=\lambda_{\mathrm{i}}\left(1+\lambda_{\mathrm{i}}\right)^{-1}$, onde $\lambda_{\mathrm{i}}$ é a i-ésima raiz característica de $\mathrm{HE}^{-1}$, essas estatísticas são dadas por:

1. $\mathbf{P}=\sum_{\mathrm{i}=1}^{\mathrm{s}} \theta_{\mathrm{i}}:$ Traço de Pillai

2. $\Delta=\prod_{\mathrm{i}=1}^{\mathrm{s}}\left(1-\theta_{\mathrm{i}}\right):$ Lambda de Wilks onde $\mathrm{s}=\min (\mathrm{t}-1, \mathrm{~g}-1)$, onde $\mathrm{t}$ são os tempos e $\mathrm{g}$ os tratamentos.

3. $\mathrm{T}=\sum_{\mathrm{i}=1}^{\mathrm{s}} \theta_{\mathrm{i}}\left(1-\theta_{\mathrm{i}}\right)^{-1}:$ Traço de Lawley-Hotelling

4. $\theta_{\mathrm{S}}=\max \left(\theta_{\mathrm{i}}\right):$ Roy

As distribuições exatas dessas estatísticas, sob a hipótese nula, dependem unicamente dos parâmetros $\mathrm{m}_{1}=(|(\mathrm{t}-1)-(\mathrm{g}-1)|-1) / 2$ e $\mathrm{m}_{2}=(\mathrm{n}-\mathrm{g}-(\mathrm{t}-1)-1) / 2$.

Esses testes não requerem a condição de $\mathrm{H}-\mathrm{F}$, pois são baseados em uma matriz de covariâncias sem estrutura. As estimativas da matriz de covariâncias podem ser obtidas pelo método dos momentos.

Os quatro testes multivariados podem produzir diferentes níveis descritivos. Em geral, a ordem de preferência em termos de poder é Traço de Pillai, Lambda de Wilks, Traço de Lawley-Hotelling e Roy. Entretanto, Lambda de Wilks é o teste mais comumente usado.

Uma desvantagem da análise multivariada, segundo Meredith \& Stehman (1991), é a falta de poder para estimar os parâmetros da matriz de covariâncias, isto quando $\mathrm{t}$ (número de ocasiões medidas ou tempos) é grande e $\mathrm{n}$ é pequeno.

Sob a condição de H-F, os testes univariados para o efeito intra-indivíduos são usualmente mais poderosos que os testes multivariados, proporcionando uma maior probabilidade de detectar efeitos significativos, quando esses realmente existem. 


\subsection{Modelo Univariado}

$\mathrm{Na}$ análise de variância, considerando-se que o experimento foi instalado seguindo o esquema de parcelas subdivididas no tempo (análise de medidas repetidas), tem-se o seguinte modelo matemático sugerido por Vonesh \& Chinchilli (1997), onde se acrescenta ao modelo (3) um novo termo de erro:

$$
\mathrm{y}_{\mathrm{ijk}}=\mu+\beta_{\mathrm{i}}+\tau_{\mathrm{j}}+(\beta \tau)_{\mathrm{ij}}+\gamma_{\mathrm{k}}+(\tau \gamma)_{\mathrm{jk}}+\mathrm{e}_{\mathrm{ijk}}
$$

onde:

$\mathrm{y}_{\mathrm{j} \mathrm{jk}}$ : é o valor observado para a variável resposta no k-ésimo tempo para o j-ésimo tratamento no i-ésimo bloco;

$\mu$ : $\quad$ é uma constante inerente a todas as observações;

$\beta_{\mathrm{i}}$ : é o efeito do i-ésimo bloco;

$\tau_{\mathrm{j}}: \quad$ é o efeito do j-ésimo tratamento;

$(\beta \tau)_{\mathrm{ij}}$ : é o efeito aleatório devido a interação do i-ésimo bloco com o j-ésimo tratamento;

$\gamma_{\mathbf{k}}$ : é o efeito do k-ésimo tempo observado;

$(\tau \gamma)_{\mathrm{jk}}$ : é o efeito da interação entre o j-ésimo tratamento com o k-ésimo tempo;

$\mathrm{e}_{\mathrm{ijk}}$ : é o erro aleatório correspondente às observações do k-ésimo tempo para o jésimo tratamento no i-ésimo bloco (variação do acaso sobre as observações), supostos homocedásticos, independentes e normalmente distribuídos.

onde:

$\mathrm{i}=1, \ldots, \mathrm{b}$ é o índice para níveis do fator blocos;

$\mathrm{j}=1, \ldots, \mathrm{g}$ é o índice para níveis do fator entre indivíduos (tratamentos);

$\mathrm{k}=1, \ldots, \mathrm{t}$ é o índice para níveis do fator intra-indivíduos (tempos).

Steel \& Torrie (1960), sugerem que, quando o delineamento for aleatorizado em blocos, a interação entre blocosxtempos, além do efeito de blocos, seja 
isolada e acrescentada ao modelo (5), por considerar que existe grande probabilidade dessa interação ser significativa.

Lima (1996) salienta que, geralmente, blocos são construídos somente para controlar a heterogeneidade entre as unidades experimentais e não se espera uma interação significativa entre a interação blocos $\times$ tempos.

O esquema de análise de variância e teste $\mathrm{F}$ para os fatores de interesse do modelo (5) é o seguinte:

Tabela 1. Análise da Variância, Esperanças dos Quadrados Médios e Teste F.

\begin{tabular}{lccccc}
\hline Causas de Variação & G.L. & S.Q. & Q.M. & E[Q.M.] & F \\
\hline$\beta$ & $(\mathrm{b}-1)$ & $\mathrm{SQ}_{1}$ & $\mathrm{QM}_{1}$ & $\sigma_{\mathrm{e}}^{2}+\mathrm{t} \sigma_{\beta \tau}^{2}+\mathrm{gt \sigma} \sigma_{\beta}^{2}$ & \\
$\tau$ & $(\mathrm{g}-1)$ & $\mathrm{SQ}_{2}$ & $\mathrm{QM}_{2}$ & $\sigma_{\mathrm{e}}^{2}+\mathrm{t} \sigma_{\beta \tau}^{2}+\phi_{1}$ & $\mathrm{QM}_{2} / \mathrm{QM}_{3}$ \\
$(\beta \tau)$ & $(\mathrm{b}-1)(\mathrm{g}-1)$ & $\mathrm{SQ}_{3}$ & $\mathrm{QM}_{3}$ & $\sigma_{\mathrm{e}}^{2}+\sigma_{\beta \tau}^{2}$ & \\
$\gamma$ & $(\mathrm{t}-1)$ & $\mathrm{SQ}_{4}$ & $\mathrm{QM}_{4}$ & $\sigma_{\mathrm{e}}^{2}+\phi_{2}$ & $\mathrm{QM}_{4} / \mathrm{QM}_{6}$ \\
$(\tau \gamma)$ & $(\mathrm{g}-1)(\mathrm{t}-1)$ & $\mathrm{SQ}_{5}$ & $\mathrm{QM}_{5}$ & $\sigma_{\mathrm{e}}^{2}+\phi_{3}$ & $\mathrm{QM}_{5} / \mathrm{QM}_{6}$ \\
Resíduo & $(\mathrm{n}-\mathrm{g})(\mathrm{t}-1)$ & $\mathrm{SQ}_{6}$ & $\mathrm{QM}_{6}$ & $\sigma_{\mathrm{e}}^{2}$ & \\
\hline Total corrigido & $\mathrm{nt}-1$ & $\mathrm{SQ}_{7}$ & $\mathrm{QM}_{7}$ & & \\
\hline
\end{tabular}

onde

$\phi_{1}=\mathrm{bt} \frac{\sum_{\mathrm{j}=1}^{\mathrm{g}} \tau_{\mathrm{j}}^{2}}{(\mathrm{~g}-1)}, \quad \phi_{2}=\mathrm{gb} \frac{\sum_{\mathrm{k}=1}^{\mathrm{t}} \gamma_{\mathrm{k}}^{2}}{(\mathrm{t}-1)} \mathrm{e} \quad \phi_{3}=\mathrm{b} \frac{\sum_{\mathrm{j}=1}^{\mathrm{g}} \sum_{\mathrm{k}=1}^{\mathrm{t}}(\tau \gamma)_{\mathrm{jk}}^{2}}{(\mathrm{~g}-1)(\mathrm{t}-1)}$.

Nesse caso, utilizando-se a análise univariada, as hipóteses de interesse testadas são:

a) $\mathrm{A}$ razão $\mathrm{QM}_{2} / \mathrm{QM}_{3}$ testa a hipótese:

$$
\begin{aligned}
& H_{0}: \tau_{1}=\tau_{2}=\cdots=\tau_{g}=0 . \\
& H_{a}: \text { Pelo menos um } \tau_{j} \neq 0 .
\end{aligned}
$$


b) A razão $\mathrm{QM}_{4} / \mathrm{QM}_{6}$ testa a hipótese:

$$
\mathrm{H}_{0}: \gamma_{1}=\gamma_{2}=\cdots=\gamma_{\mathrm{t}}=0
$$

$\mathrm{H}_{\mathrm{a}}$ : Pelo menos um $\gamma_{\mathrm{k}} \neq 0$.

c) A razão $\mathrm{QM}_{5} / \mathrm{QM}$ testa a hipótese:

$$
\begin{aligned}
& \mathrm{H}_{0}:(\tau \gamma)_{11}=(\tau \gamma)_{12}=\cdots=(\tau \gamma)_{\mathrm{gt}}=0 . \\
& \mathrm{H}_{\mathrm{a}}: \text { Pelo menos um }(\tau \gamma)_{\mathrm{jk}} \neq 0 .
\end{aligned}
$$

Geralmente, considera-se como nível mínimo para a rejeição da hipótese $\mathrm{H}_{0}, 0,05$, ou seja, sempre que o valor da probabilidade do teste $\mathrm{F}$ for menor ou igual a 0,05, aceita-se que há diferença entre os níveis dos fatores. A interpretação dos testes deve ser iniciada pelas interações, considerando-se primeiramente a interação dupla. E, se a interação não for significativa então consideram-se os testes para os efeitos principais.

Podem-se obter as seguintes interpretações geométricas, caso as hipóteses testadas sejam significativas (Figuras 1, 2 e 3). Foram usados valores arbitrários para as médias, apenas por questões didáticas.

a) Quando a hipótese $H_{0}: \tau_{1}=\tau_{2}=\cdots=\tau_{\mathrm{g}}=0$ é rejeitada, indica que existem diferenças verticais entre as curvas de grupos ou tratamentos;

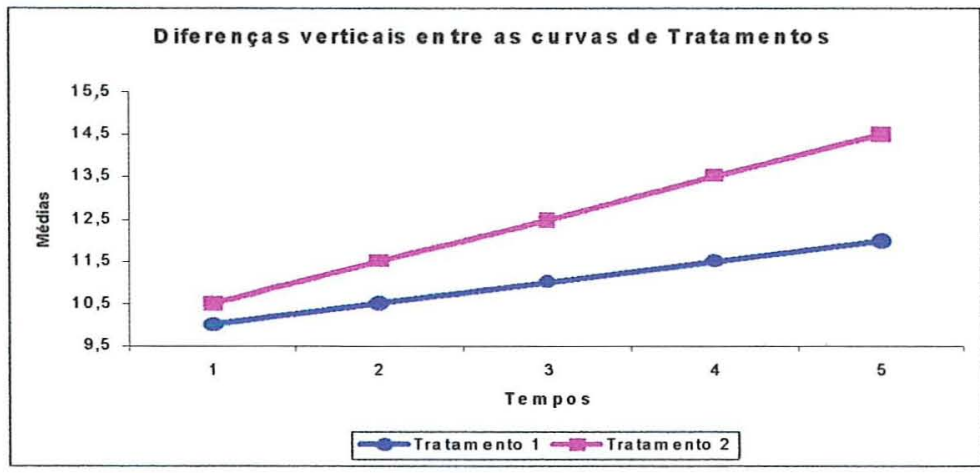

Figura 1. Diferenças verticais entre as curvas de Tratamentos. 
b) Quando a hipótese $H_{0}: \gamma_{1}=\gamma_{2}=\cdots=\gamma_{t}=0$ é rejeitada, indica que as curvas não são constantes no tempo;

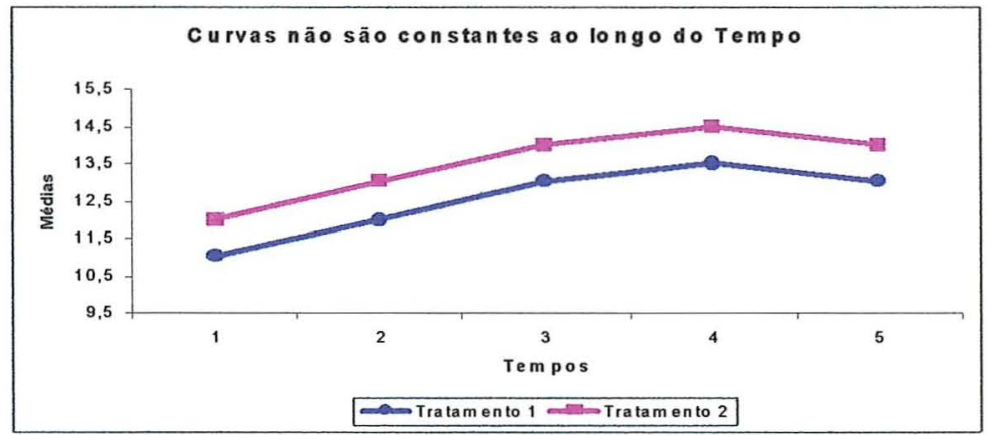

Figura 2. Curvas não são constantes ao longo do Tempo.

c) Quando a hipótese $\mathrm{H}_{0}:(\tau \gamma)_{11}=(\tau \gamma)_{12}=\cdots=(\tau \gamma)_{\mathrm{gt}}=0$ é rejeitada, indica que a forma das curvas diferem entre os tratamentos ao longo do tempo.

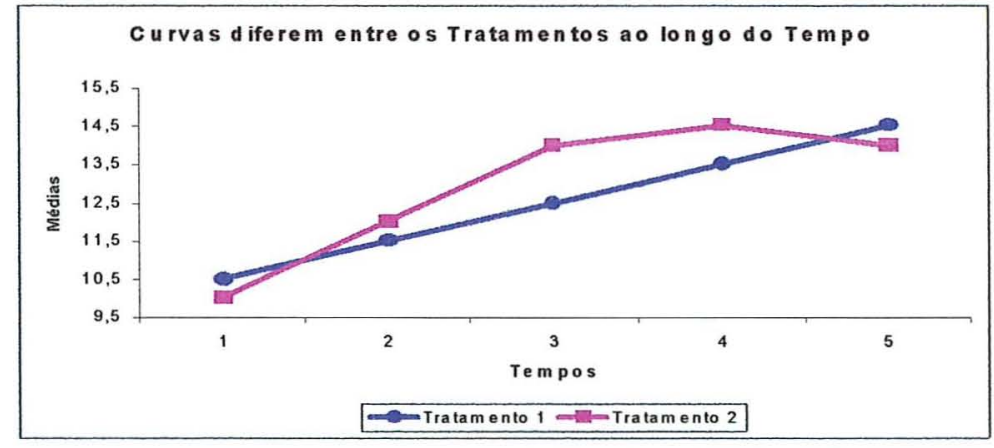

Figura 3. Curvas diferem entre os Tratamentos ao longo do Tempo. 


\subsection{Correções para os Números de Graus de Liberdade}

Box (1945 a,b) foi o primeiro a sugerir a correção para o número de graus de liberdade, a fim de se obter uma aproximação da distribuição $F$, quando a matriz de covariâncias dos erros intra-indivíduos não leva em conta a suposição de variância constante .

Geisser \& Greenhouse (1958), e Huynh \& Feldt (1976) também propuseram ajustes para o número de graus de liberdade do teste $\mathrm{F}$, para o fator de erro intra-indivíduos. Essas correções foram baseadas no trabalho de Box (1945 a, b), sendo a correção de HUYNH-FELDT uma simples função da correção de GEISSERGREENHOUSE.

De acordo com Kuehl (1994), para a obtenção das correções de GEISSER-GREENHOUSE e HUYNH-FELDT considera-se $s_{i j}$ a i-ésima linha e a jésima coluna da matriz de covariâncias amostral $S_{(t \times t)}$, como sendo o erro experimental intra-individuos. Escolhem-se $q=(t-1)$ contrastes ortogonais normalizados, sobre $t$ medidas repetidas e toma-se a matriz $\mathbf{C}_{(\mathrm{q} \times t)}$ onde as linhas são contrastes ortogonais normalizados nas $\mathrm{t}$ medidas repetidas. Calculando-se a matriz

$$
{ }_{q} A_{q}=\mathrm{CSC}^{\prime} \text {, }
$$

com $a_{\mathrm{ij}}$ definindo um elemento genérico, pode-se obter então o ajuste de GEISSERGREENHOUSE $\hat{\varepsilon}$

$$
\hat{\varepsilon}=\frac{\left(\sum_{i=1}^{q} a_{i i}\right)^{2}}{(t-1) \sum_{i=1}^{q} \sum_{j=1}^{q} a_{i j}^{2}},
$$

e o ajuste de HUYNH-FELDT $\widetilde{\varepsilon}$

$$
\widetilde{\varepsilon}=\frac{(N(t-1) \hat{\varepsilon}-2)}{(t-1)[(t-1)(b-1)-(t-1) \hat{\varepsilon}]},
$$


onde $\mathrm{N}$ é o número total de indivíduos, $\mathrm{g}$ é o número de níveis do fator da parcela e t é o número de medidas repetidas (tempos).

Por exemplo, dada uma estatística F, baseada em mínimos quadrados, com números de graus de liberdade $v_{1}$ e $v_{2}$, o ajuste para esses números de graus de liberdade seria $\varepsilon v_{1}$ e $\varepsilon v_{2}$.

A correção de HUYNH-FELDT ( $\widetilde{\varepsilon}$ ) é mais liberal do que a correção de GEISSER-GREENHOUSE ( $\hat{\varepsilon}$ ). Porém, a correção de HUYNH-FELDT não deve ser usada se $\widetilde{\varepsilon} \geq 1$, mas é recomendada quando $\hat{\varepsilon} \geq 0,75$ para reduzir o vício de grandes valores da correção de GEISSER-GREENHOUSE, Huynh \& Feldt (1976) e Huynh (1978).

Kirk (1995) discute que, quando a suposição de esfericidade é satisfeita, os fatores de correção $\hat{\varepsilon}$ e $\widetilde{\varepsilon}$ são iguais a 1, caso contrário, são menores, mas devem ter um mínimo de $\frac{1}{(t-1)}$.

De acordo com Muller \& Barton (1989), com a correção do número de graus de liberdade obtêm-se testes mais conservativos, que são limitados a assegurar que $\alpha$ esteja abaixo de um certo nível. Isso para casos em que um teste aproximado não é desejável, e casos no qual a matriz de covariâncias é diferente de tratamento para tratamento.

Quanto à escolha de qual correção para o número de graus de liberdade usar, Muller \& Barton (1989), depois de vários estudos com simulações, verificando o poder dos testes quando as correções são utilizadas, sugerem que a correção de GEISSER-GREENHOUSE seja utilizada já que o teste produz aceitável controle do erro tipo I enquanto maximiza o poder. Mas, segundo Huynh \& Feldt (1976) a correção de GEISSER-GREENHOUSE tem a desvantagem de superestimar o verdadeiro nível de significância.

Sendo assim, a análise univariada é recomendada, mesmo que a condição de H-F para a matriz de covariâncias não seja satisfeita, porém, utilizando-se a correção 
de HUYNH-FELDT, desde que o teste de esfericidade seja significativo, com um nível de probabilidade entre 0,01 e 0,05 .

\subsection{Modelo Misto}

Como já visto anteriormente, no item 2.3 , em análise de medidas repetidas no tempo, o modelo univariado requer certa suposição referente à estrutura da matriz de covariâncias, que quando não é satisfeita leva a testes aproximados.

Com relação ao modelo multivariado, item 2.2 , por apresentar uma estrutura geral para a matriz de covariâncias, é de dificil utilização quando os dados são desbalanceados. Para contornar essa situação, uma técnica alternativa aos modelos uni e multivariados, quando a suposição da matriz de covariâncias não é satisfeita, com relação à condição de H-F, é a análise com modelos mistos que são uma extensão do modelo linear geral.

Os modelos mistos englobam análise de curvas de crescimento, ou curvas polinomiais, que levam em conta a estrutura da matriz de covariâncias que melhor explica o comportamento das observações, tendo a vantagem de ajustar modelos que reduzem o número de parâmetros, Von Ende (1993), Littell et al. (1998).

De acordo com Kshirsagar \& Smith (1995) modelos de curvas de crescimento ou polinomiais são mais gerais do que o modelo usual de medidas repetidas. A diferença entre eles está no interesse do pesquisador, por exemplo, em modelos de medidas repetidas o interesse básico é detectar diferença entre os tratamentos no decorrer do tempo. Já em modelos de curvas de crescimento, o objetivo básico é estimar e predizer os efeitos de tratamentos em algum tempo.

Em modelos de curvas de crescimento, ou polinomiais, parte-se do princípio que existe uma relação funcional entre os efeitos de tratamentos e o tempo de aplicação, e que esta relação pode ser modelada. A função pode ser aproximada por um 
polinômio, devendo os coeficientes dessa representação polinomial, bem como variâncias e covariâncias, serem estimadas através dos dados.

A equação polinomial de crescimento pode ser usada para descrever aproximadamente outras curvas, sendo que a precisão será maior ou menor, adicionandose ou retirando-se termos de maior ordem.

Esses modelos permitem a utilização de várias estruturas de covariâncias no processo de modelagem.

Os modelos mistos, em dois estágios, abordados por Laird \&Ware (1982), Ware (1985), Jennrich \& Schluchter (1986) e Diggle et al. (1998), consideram os efeitos fixos no primeiro estágio para obtenção da curva polinomial média, e no segundo estágio permitem diferentes curvas para cada indivíduo.

O modelo é expresso da seguinte forma:

$$
y_{i}=X_{i} \beta+Z_{i} \gamma_{i}+e_{i}
$$

onde:

$\mathrm{y}_{\mathrm{i}}$ : $\quad$ é um vetor coluna $\left(\mathrm{n}_{\mathrm{i}} \times 1\right)$, das $\mathrm{n}_{\mathrm{i}}$ observações tomadas da unidade $\mathrm{i}$ ao longo do tempo ou condição de avaliação;

$\quad \beta$ : $\quad$ é um vetor $(\mathrm{p} \times 1)$ de parâmetros fixos desconhecidos, onde a dimensão $\mathrm{p}$ é fixa para qualquer unidade;

$\mathrm{X}_{\mathrm{i}}$ : $\quad$ é uma matriz $\left(\mathrm{n}_{\mathrm{i}} \mathrm{x} \mathrm{p}\right)$ que faz a seleção dos elementos do vetor $\beta$;

$\gamma_{\mathrm{i}}$ : é um vetor $(\mathrm{g} \times 1)$ de g efeitos aletórios desconhecidos, onde $\gamma_{\mathrm{i}} \cap \mathrm{N}(0, \mathrm{G})$;

$\mathrm{Z}_{\mathrm{i}}$ : $\quad$ é uma matriz $\left(\mathrm{n}_{\mathrm{i}} \times \mathrm{g}\right)$ que faz a seleção dos elementos do vetor $\gamma_{\mathrm{i}}$;

$e_{i}$ : é um vetor $\left(n_{i} \times 1\right)$ de erros aleatórios, onde $e_{i} \cap N\left(0, R_{i}\right)$.

A matriz $G$ é positiva semidefinida e $R_{i}\left(t_{i} \times t_{i}\right)$ é uma matriz positiva definida. Os $\gamma_{i}$ são independentes entre si e dos erros $e_{i}$.

Assim

$$
\mathrm{y}_{\mathrm{i}} \cap \mathrm{N}\left(\mathrm{X}_{\mathrm{i}} \beta, \Sigma_{\mathrm{i}}\right) \text {, }
$$

onde $\Sigma_{\mathbf{i}}=Z_{\mathbf{i}} G Z_{i}^{\prime}+R_{i}$. 
Deve-se ressaltar que os elementos do vetor $\gamma_{\mathrm{i}}$ consistem de parâmetros individuais, que representam as diferenças entre a curva do tratamento e a curva de cada individuo, e são inseridos no modelo com o objetivo de proporcionar um melhor ajuste.

As estimativas $\hat{\beta}$ e $\hat{\gamma}$ são obtidas por máxima verossimilhança ou máxima verossimilhança restrita através de algum método iterativo (Laird \& Ware, 1982).

De acordo com Guimarães (1994) e Matsushita (1994) as estimativas de máxima verossimilhança são dadas por

$$
\hat{\beta}=\left(\sum_{i=1}^{n} X_{i}^{\prime} \hat{\Sigma}_{i}^{-1} X_{i}\right)^{-1}\left(\sum_{i=1}^{n} X_{i}^{\prime} \hat{\Sigma}_{i}^{-1} y_{i}\right)
$$

e

$$
\hat{\gamma}=\hat{G} Z_{i}^{\prime} \hat{\Sigma}_{i}^{-1}\left(y_{i}-X_{i} \hat{\beta}\right)
$$

Detalhes sobre os estimadores dos parâmetros que envolvem máxima verossimilhança e máxima verossimilhança restrita serão apresentados no decorrer do texto.

A vantagem de se trabalhar com modelos mistos, que envolvem curvas de crescimento ou polinômios, é a possibilidade de poder optar pela estrutura de covariâncias que melhor represente os dados.

\subsection{Estruturas da Matriz de Covariâncias}

Nos itens anteriores foram apresentadas três técnicas para analisar um experimento com medidas repetidas no tempo, onde as estruturas para a matriz de covariâncias são diferentes. No caso multivariado, a estrutura da matriz de covariâncias é da forma mais geral possível, ou seja, as variâncias e covariâncias podem ser diferentes. No caso univariado faz-se a exigência de que a estrutura da matriz de covariâncias satisfaça a condição de $\mathrm{H}-\mathrm{F}$, já discutida anteriormente, no item 2 . $\mathrm{E}$, finalmente, o caso em que se trabalha com modelos mistos, onde a estrutura da matriz de covariâncias pode 
ser modelada da forma que melhor represente os dados, ou seja, pode levar em consideração se os dados são independentes, dependentes, correlacionados ou ainda apresentar outra relação que a matriz de covariâncias usual não consegue explicar.

Por esse motivo, a seguir, serão apresentadas algumas das estruturas da matriz de covariâncias mais utilizadas, e que já se encontram implementadas no "software" SAS:

1. Componente de Variância (VC):

Variâncias iguais e observações independentes.

$$
\Sigma=\left[\begin{array}{cccc}
\sigma^{2} & 0 & 0 & 0 \\
0 & \sigma^{2} & 0 & 0 \\
0 & 0 & \sigma^{2} & 0 \\
0 & 0 & 0 & \sigma^{2}
\end{array}\right]
$$

\section{Simetria Composta (CS):}

Igualdade de variâncias e covariâncias, ou seja, covariâncias constantes entre quaisquer observações de uma mesma unidade devido a erros independentes.

$$
\Sigma=\left[\begin{array}{cccc}
\left(\sigma^{2}+\sigma_{1}^{2}\right) & \sigma_{1}^{2} & \sigma_{1}^{2} & \sigma_{1}^{2} \\
\sigma_{1}^{2} & \left(\sigma^{2}+\sigma_{1}^{2}\right) & \sigma_{1}^{2} & \sigma_{1}^{2} \\
\sigma_{1}^{2} & \sigma_{1}^{2} & \left(\sigma^{2}+\sigma_{1}^{2}\right) & \sigma_{1}^{2} \\
\sigma_{1}^{2} & \sigma_{1}^{2} & \sigma_{1}^{2} & \left(\sigma^{2}+\sigma_{1}^{2}\right)
\end{array}\right]
$$

\section{Sem Estrutura (UN):}

Todas as variâncias e as covariâncias podem ser desiguais. Especifica uma matriz completamente geral, parametrizada diretamente em termos de variâncias e covariâncias. As variâncias são restritas a valores não negativos e as covariâncias não têm restrições. 


$$
\Sigma=\left[\begin{array}{llll}
\sigma_{11} & \sigma_{12} & \sigma_{13} & \sigma_{14} \\
\sigma_{12} & \sigma_{22} & \sigma_{23} & \sigma_{24} \\
\sigma_{13} & \sigma_{23} & \sigma_{33} & \sigma_{34} \\
\sigma_{14} & \sigma_{24} & \sigma_{34} & \sigma_{44}
\end{array}\right]
$$

\section{4. "Banded" (UN(q)):}

Variâncias desiguais e covariâncias arbitrárias para cada defasagem q-1 (onde q é o número de parâmetros de covariâncias) e zeros para defasagens maiores. Por exemplo $\mathrm{q}=3$.

$$
\Sigma=\left[\begin{array}{cccc}
\sigma_{1} & \sigma_{5} & \sigma_{8} & 0 \\
\sigma_{5} & \sigma_{2} & \sigma_{6} & \sigma_{9} \\
\sigma_{8} & \sigma_{6} & \sigma_{3} & \sigma_{7} \\
0 & \sigma_{9} & \sigma_{7} & \sigma_{4}
\end{array}\right]
$$

5. Diagonal Principal "Banded" (UN(1)):

Variâncias desiguais e covariâncias nulas.

$$
\Sigma=\left[\begin{array}{cccc}
\sigma_{1}^{2} & 0 & 0 & 0 \\
0 & \sigma_{2}^{2} & 0 & 0 \\
0 & 0 & \sigma_{3}^{2} & 0 \\
0 & 0 & 0 & \sigma_{4}^{2}
\end{array}\right]
$$

6. Auto-regressiva de $1^{\text {a. }}$ Ordem (AR(1)):

Dados de séries temporais igualmente espaçados e correlações diminuindo exponencialmente, ou seja, a covariância entre duas observações decresce à medida em que aumenta o intervalo de tempo entre elas, onde o parâmetro auto-regressivo é $\rho$, que para um processo estacionário assume $|\rho|<1$. 


$$
\Sigma=\sigma^{2}\left[\begin{array}{cccc}
1 & \rho & \rho^{2} & \rho^{3} \\
\rho & 1 & \rho & \rho^{2} \\
\rho^{2} & \rho & 1 & \rho \\
\rho^{3} & \rho^{2} & \rho & 1
\end{array}\right]
$$

\section{Toeplitz (TOEP):}

Dados de séries temporais igualmente espaçados e correlação arbitrária para cada defasagem.

$$
\Sigma=\left[\begin{array}{llll}
\sigma^{2} & \sigma_{1} & \sigma_{2} & \sigma_{3} \\
\sigma_{1} & \sigma^{2} & \sigma_{1} & \sigma_{2} \\
\sigma_{2} & \sigma_{1} & \sigma^{2} & \sigma_{1} \\
\sigma_{3} & \sigma_{2} & \sigma_{1} & \sigma^{2}
\end{array}\right]
$$

8. "Banded" Toeplitz (TOEP(q)):

Dados de séries temporais igualmente espaçados, correlações arbitrárias para cada defasagem $\mathrm{q}-1$ e zeros para as defasagens mais distantes. Por exemplo $\mathrm{q}=2$.

$$
\Sigma=\left[\begin{array}{cccc}
\sigma^{2} & \sigma_{1} & 0 & 0 \\
\sigma_{1} & \sigma^{2} & \sigma_{1} & 0 \\
0 & \sigma_{1} & \sigma^{2} & \sigma_{1} \\
0 & 0 & \sigma_{1} & \sigma^{2}
\end{array}\right]
$$

9. Auto-regressiva de $1^{\text {a. }}$ Ordem Heterogênea (ARH(1)):

Dados de séries temporais com variâncias e covariâncias desiguais, onde $\rho$ é o parâmetro auto-regressivo satisfazendo $|\rho|<1$.

$$
\Sigma=\left[\begin{array}{cccc}
\sigma_{1}^{2} & \sigma_{1} \sigma_{2} \rho & \sigma_{1} \sigma_{3} \rho^{2} & \sigma_{1} \sigma_{4} \rho^{3} \\
\sigma_{2} \sigma_{1} \rho & \sigma_{2}^{2} & \sigma_{2} \sigma_{3} \rho & \sigma_{2} \sigma_{4} \rho^{2} \\
\sigma_{3} \sigma_{1} \rho^{2} & \sigma_{3} \sigma_{2} \rho & \sigma_{3}^{2} & \sigma_{3} \sigma_{4} \rho \\
\sigma_{4} \sigma_{1} \rho^{3} & \sigma_{4} \sigma_{2} \rho^{2} & \sigma_{4} \sigma_{3} \rho & \sigma_{4}^{2}
\end{array}\right]
$$


10. Auto-regressiva de $1^{\text {a. }}$ Ordem Médias Móveis $(\operatorname{ARMA}(1,1))$ :

Dados de séries temporais com parâmetro auto-regressivo $\rho$, componente de médias móveis $\gamma$, sendo $\sigma^{2}$ a variância residual.

$$
\Sigma=\sigma^{2}\left[\begin{array}{rrrr}
1 & \gamma & \gamma \rho & \gamma \rho^{2} \\
\gamma & 1 & \gamma & \gamma \rho \\
\gamma \rho & \gamma & 1 & \gamma \\
\gamma \rho^{2} & \gamma \rho & \gamma & 1
\end{array}\right]
$$

11. Simetria Composta Heterogênea (CSH):

Parâmetros de variâncias diferentes para cada elemento da diagonal principal e raiz quadrada desses parâmetros nos elementos fora da diagonal principal, sendo $\sigma_{i}^{2}$ o i-ésimo parâmetro da variância e $\rho$ o parâmetro de correlação satisfazendo $|\rho|<1$.

$$
\Sigma=\left[\begin{array}{cccc}
\sigma_{1}^{2} & \sigma_{1} \sigma_{2} \rho & \sigma_{1} \sigma_{3} \rho & \sigma_{1} \sigma_{4} \rho \\
\sigma_{2} \sigma_{1} \rho & \sigma_{2}^{2} & \sigma_{2} \sigma_{3} \rho & \sigma_{2} \sigma_{4} \rho \\
\sigma_{3} \sigma_{1} \rho & \sigma_{3} \sigma_{2} \rho & \sigma_{3}^{2} & \sigma_{3} \sigma_{4} \rho \\
\sigma_{4} \sigma_{1} \rho & \sigma_{4} \sigma_{2} \rho & \sigma_{4} \sigma_{3} \rho & \sigma_{4}^{2}
\end{array}\right]
$$

12. Estrutura Fator Analítico (FA(q)):

Especifica uma estrutura com q fatores (Jennrich \& Schluchter 1986). Essa estrutura é da forma $\Lambda \Lambda^{\prime}+\mathrm{D}$, onde $\Lambda$ é uma matriz retangular (t $\left.\mathrm{x} q\right)$ e $\mathrm{D}$ é uma matriz diagonal $(\mathrm{t} \times \mathrm{t})$ de variâncias únicas. Quando $\mathrm{q}>1$, os elementos no canto superior direito (elementos na i-ésima linha e j-ésima coluna com $\mathrm{j}>\mathrm{i}$ ) de $\Lambda$ são um conjunto de zeros.

$$
\Sigma=\left[\begin{array}{cccc}
\lambda_{1}^{2}+d_{1} & \lambda_{1} \lambda_{2} & \lambda_{1} \lambda_{3} & \lambda_{1} \lambda_{4} \\
\lambda_{2} \lambda_{1} & \lambda_{2}^{2}+d_{2} & \lambda_{2} \lambda_{3} & \lambda_{2} \lambda_{4} \\
\lambda_{3} \lambda_{1} & \lambda_{3} \lambda_{2} & \lambda_{3}^{2}+d_{3} & \lambda_{3} \lambda_{4} \\
\lambda_{4} \lambda_{1} & \lambda_{4} \lambda_{2} & \lambda_{4} \lambda_{3} & \lambda_{4}^{2}+d_{4}
\end{array}\right]
$$


13. Estrutura Fator Analítico (FA0(q)):

É similar a estrutura fator analítico, exceto que não inclui $\mathrm{D}$ na diagonal da matriz. Quando $\mathrm{q}<\mathrm{k}$, isto é, quando número de fatores é menor do que a dimensão da matriz, essa estrutura é definida não negativa, mas de posto incompleto.

$$
\Sigma=\left[\begin{array}{cccc}
\lambda_{1}^{2} & \lambda_{1} \lambda_{2} & \lambda_{1} \lambda_{3} & \lambda_{1} \lambda_{4} \\
\lambda_{2} \lambda_{1} & \lambda_{2}^{2} & \lambda_{2} \lambda_{3} & \lambda_{2} \lambda_{4} \\
\lambda_{3} \lambda_{1} & \lambda_{3} \lambda_{2} & \lambda_{3}^{2} & \lambda_{3} \lambda_{4} \\
\lambda_{4} \lambda_{1} & \lambda_{4} \lambda_{2} & \lambda_{4} \lambda_{3} & \lambda_{4}^{2}
\end{array}\right]
$$

14. Estrutura Fator Analítico (FA1(q)):

É similar à estrutura fator analítico, exceto que todos os elementos em D devem ser iguais.

$$
\Sigma=\left[\begin{array}{cccc}
\lambda_{1}^{2}+\mathrm{d} & \lambda_{1} \lambda_{2} & \lambda_{1} \lambda_{3} & \lambda_{1} \lambda_{4} \\
\lambda_{2} \lambda_{1} & \lambda_{2}^{2}+\mathrm{d} & \lambda_{2} \lambda_{3} & \lambda_{2} \lambda_{4} \\
\lambda_{3} \lambda_{1} & \lambda_{3} \lambda_{2} & \lambda_{3}^{2}+\mathrm{d} & \lambda_{3} \lambda_{4} \\
\lambda_{4} \lambda_{1} & \lambda_{4} \lambda_{2} & \lambda_{4} \lambda_{3} & \lambda_{4}^{2}+d
\end{array}\right]
$$

15. Huynh-Feldt (HF):

Essa estrutura,(HUYNH \& FELDT, 1970), é similar à simetria composta heterogênea, que tem o mesmo número de parâmetros e heterogeneidade ao longo da diagonal principal. Entretanto, a construção dos elementos fora da diagonal é feita tomando-se a média aritmética entre as variâncias e subtraindo $\lambda$, onde $\lambda$ é a diferença entre a média das variâncias e a média das covariâncias.

$$
\Sigma=\left[\begin{array}{cccc}
\sigma_{1}^{2} & \frac{\left(\sigma_{1}^{2}+\sigma_{2}^{2}\right)}{2}-\lambda & \frac{\left(\sigma_{1}^{2}+\sigma_{3}^{2}\right)}{2}-\lambda & \frac{\left(\sigma_{1}^{2}+\sigma_{4}^{2}\right)}{2}-\lambda \\
\frac{\left(\sigma_{2}^{2}+\sigma_{1}^{2}\right)}{2}-\lambda & \sigma_{2}^{2} & \frac{\left(\sigma_{2}^{2}+\sigma_{3}^{2}\right)}{2}-\lambda & \frac{\left(\sigma_{2}^{2}+\sigma_{4}^{2}\right)}{2}-\lambda \\
\frac{\left(\sigma_{3}^{2}+\sigma_{1}^{2}\right)}{2}-\lambda & \frac{\left(\sigma_{3}^{2}+\sigma_{2}^{2}\right)}{2}-\lambda & \sigma_{3}^{2} & \frac{\left(\sigma_{3}^{2}+\sigma_{4}^{2}\right)}{2}-\lambda \\
\frac{\left(\sigma_{4}^{2}+\sigma_{1}^{2}\right)}{2}-\lambda & \frac{\left(\sigma_{4}^{2}+\sigma_{2}^{2}\right)}{2}-\lambda & \frac{\left(\sigma_{4}^{2}+\sigma_{3}^{2}\right)}{2}-\lambda & \sigma_{4}^{2}
\end{array}\right]
$$


16. Primeira Antedependência (ANTE(1)):

Estrutura de antedependência de $1^{\text {a. }}$ ordem, parâmetros de variâncias diferentes para cada elemento da diagonal, sendo os elementos fora da diagonal principal funções de variâncias e do k-ésimo parâmetro de autocorrelação, satisfazendo $\left|\rho_{k}\right|<1$.

$$
\Sigma=\left[\begin{array}{cccc}
\sigma_{1}^{2} & \sigma_{1} \sigma_{2} \rho_{1} & \sigma_{1} \sigma_{3} \rho_{1} \rho_{2} & \sigma_{1} \sigma_{4} \rho_{1} \rho_{2} \rho_{3} \\
\sigma_{2} \sigma_{1} \rho_{1} & \sigma_{2}^{2} & \sigma_{2} \sigma_{3} \rho_{2} & \sigma_{2} \sigma_{4} \rho_{2} \rho_{3} \\
\sigma_{3} \sigma_{1} \rho_{2} \rho_{1} & \sigma_{3} \sigma_{2} \rho_{2} & \sigma_{3}^{2} & \sigma_{3} \sigma_{4} \rho_{3} \\
\sigma_{4} \sigma_{1} \rho_{3} \rho_{2} \rho_{1} & \sigma_{4} \sigma_{2} \rho_{3} \rho_{2} & \sigma_{4} \sigma_{3} \rho_{3} & \sigma_{4}^{2}
\end{array}\right]
$$

\section{Toeplitz Heterogênea (TOEPH(q)):}

Dados de séries temporais igualmente espaçados, com parâmetros de variâncias diferentes para cada elemento da diagonal, sendo os elementos fora da diagonal principal funções de variâncias e do k-ésimo parâmetro de autocorrelação $\left(\left|\rho_{\mathrm{k}}\right|<1\right)$ para cada defasagem $\mathrm{q}-1$, e zeros para as últimas defasagens. Por exemplo, $\mathrm{q}=3$.

$$
\Sigma=\left[\begin{array}{cccc}
\sigma_{1}^{2} & \sigma_{1} \sigma_{2} \rho_{1} & \sigma_{1} \sigma_{3} \rho_{2} & 0 \\
\sigma_{2} \sigma_{1} \rho_{1} & \sigma_{2}^{2} & \sigma_{2} \sigma_{3} \rho_{1} & \sigma_{2} \sigma_{4} \rho_{2} \\
\sigma_{3} \sigma_{1} \rho_{2} & \sigma_{3} \sigma_{2} \rho_{1} & \sigma_{3}^{2} & \sigma_{3} \sigma_{4} \rho_{1} \\
0 & \sigma_{4} \sigma_{2} \rho_{2} & \sigma_{4} \sigma_{3} \rho_{1} & \sigma_{4}^{2}
\end{array}\right]
$$

\section{Correlação sem Estrutura (UNR(q)):}

Especifica uma matriz de covariâncias completamente geral em termos de variâncias e correlações. Essa estrutura ajusta o mesmo modelo que o tipo sem Estrutura, mas com diferente parametrização. $\mathrm{O}$ i-ésimo parâmetro de variância é $\sigma_{i}^{2}$. $O$ parâmetro de $\rho_{\mathrm{jk}}$, é a correlação entre a j-ésima e a k-ésima medida satisfazendo $\left(\left|\rho_{\mathrm{jk}}\right|<1\right)$. Por exemplo, $q=3$.

$$
\Sigma=\left[\begin{array}{cccc}
\sigma_{1}^{2} & \sigma_{1} \sigma_{2} \rho_{21} & \sigma_{1} \sigma_{3} \rho_{31} & \sigma_{1} \sigma_{4} \rho_{41} \\
\sigma_{2} \sigma_{1} \rho_{21} & \sigma_{2}^{2} & \sigma_{2} \sigma_{3} \rho_{32} & \sigma_{2} \sigma_{4} \rho_{42} \\
\sigma_{3} \sigma_{1} \rho_{31} & \sigma_{3} \sigma_{2} \rho_{32} & \sigma_{3}^{2} & \sigma_{3} \sigma_{4} \rho_{43} \\
\sigma_{4} \sigma_{1} \rho_{41} & \sigma_{4} \sigma_{2} \rho_{42} & \sigma_{4} \sigma_{3} \rho_{43} & \sigma_{4}^{2}
\end{array}\right]
$$


19. Potência (SP(POW)(c-list)):

Covariâncias são funções da distância Euclidiana entre os vetores especificados pelas coordenadas. O parâmetro c-list associado à essa estrutura espacial, corresponde aos nomes das variáveis numéricas no conjunto de dados. Essas variáveis são usadas como coordenadas das observações no espaço, sendo essa estrutura utilizada para dados de séries temporais desigualmente espaçados.

$$
\Sigma=\left[\begin{array}{cccc}
1 & \rho^{d_{12}} & \rho^{d_{13}} & \rho^{d_{14}} \\
\rho^{d_{12}} & 1 & \rho^{d_{23}} & \rho^{d_{24}} \\
\rho^{d_{13}} & \rho^{d_{23}} & 1 & \rho^{d_{34}} \\
\rho^{d_{14}} & \rho^{d_{24}} & \rho^{d_{34}} & 1
\end{array}\right]
$$

20. Produtos Diretos (UN@AR(1), UN@CS e UN@UN):

Essas estruturas especificam produtos diretos (Kronecker), designados para medidas repetidas multivariadas. São construídas fazendo o produto de Kronecker de uma matriz sem estrutura (modelando covariâncias sobre observações multivariadas), com uma matriz de covariâncias adicional modelando covariâncias sobre tempo ou outro fator.

\subsection{Seleção do Modelo}

Tendo em vista que o número de estruturas de covariâncias é elevado, um dos principais objetivos da análise é o de encontrar um modelo que melhor represente os dados, dentre vários modelos possiveis.

Duas técnicas serão apresentadas a seguir para auxiliar na escolha do modelo adequado. 


\subsubsection{Teste Assintótico da Razão de Verossimilhança}

Esse teste compara dois modelos estimados por máxima verossimilhança, onde um dos modelos é uma versão restrita do outro, ou seja, um modelo tem $r$ parâmetros adicionais. $\mathrm{O}$ teste irá verificar se esses parâmetros adicionais melhoram significativamente o modelo. Definindo-se $\ell_{1}$ o valor de $\ell=(-2 \ln$ da função de verossimilhança) para o modelo com o menor número de parâmetros e $\ell_{2}$ para o modelo com maior número de parâmetros, ou seja, para o modelo com r parâmetros extras, a hipótese a ser testada é a de que os dois modelos são iguais (os parâmetros extras são iguais a zero). A diferença entre os valores $\ell_{1}$ e $\ell_{2}$ é assintoticamente distribuída como uma Qui-Quadrado com r graus de liberdade,

$$
\ell_{1}-\ell_{2} \sim \chi_{\mathrm{r}}^{2} \text {. }
$$

A desvantagem desse teste, embora seja bastante eficaz, é que só pode ser usado para comparar dois modelos de cada vez, sendo que um desses modelos é sempre um caso especial do outro (Guimarães, 1994) (Matsushita, 1994).

\subsubsection{Critério de Informação de Akaike (AIC)}

O AIC é baseado na teoria de decisão e penaliza os modelos com número grande de parâmetros para evitar excesso de parametrização.

O AIC pode ser definido de duas formas:

$$
\mathrm{AIC}=-2 \ell+2 \times \mathrm{p}
$$

e

$$
\mathrm{AIC}=\ell-\mathrm{p}
$$

onde:

$\ell$ é o ln da função de verossimilhança; 
$\mathrm{p}$ é o número de parâmetros da matriz de covariâncias.

A maneira como o AIC é obtido depende da forma como o "software" utilizado realiza os cálculos. Por exemplo, no SAS, quando se trabalha com o proc REG, o AIC é definido por (7). Agora, quando o proc MIXED é utilizado o AIC é definido por (8).

Para tomada de decisão sobre qual modelo será utilizado é necessário que o AIC seja calculado para todos os modelos considerados. Quando o AIC é obtido por (7), o modelo escolhido será aquele que apresentar o menor valor de AIC. O contrário ocorre quando o cálculo do AIC é realizado por (8), nesse caso o modelo escolhido será aquele com o maior valor de AIC.

\subsection{Estimação}

Em muitos casos, como já visto anteriormente nos itens 2.2 e 2.3 , as técnicas de análise clássica para medidas repetidas não podem ser utilizadas por não satisfazerem às pressuposições necessárias, ou porque algumas observações foram perdidas, ou ainda porque o delineamento é desbalanceado por alguma razão. Também tem-se algumas estruturas de covariâncias que na sua formação levam em conta fatores fixos e aleatórios, e por esse motivo, não podem ser estimados pelo Método dos Mínimos Quadrados, sendo necessário, então, utilizar o Método da Máxima Verossimilhança ou o Método da Máxima Verossimilhança Restrita. Para se obterem as estimativas de máxima verossimilhança são usados algoritmos iterativos tais como o de Newton-Raphson, Score de Fisher e o algoritmo EM. 


\subsubsection{Método da Máxima Verossimilhança (MV)}

O método da Estimação por Máxima Verossimilhança consiste em: expressar $\mathrm{L}(\theta)$ como o produtório das densidades associadas a cada observação da amostra aleatória $y_{1}, y_{2}, \ldots, y_{n}$; diferenciar parcialmente $L(\theta)$ em relação a cada componente do vetor $\theta$; igualar cada derivada a zero, e resolver o sistema de equações formadas por essas derivadas. A solução desse sistema é o vetor de Estimativas de Máxima Verossimilhança (EMV).

Desconsiderando-se os modelos apresentados anteriormente, para ilustrar o método considere-se o modelo a seguir

$$
\mathrm{y}_{\mathrm{i}}=\mathrm{X}_{\mathrm{i}} \beta+\mathrm{e}_{\mathrm{i}}
$$

onde $y_{i}$ é um vetor $t_{i} \times 1$ contendo as respostas do indivíduo $i, i=1, \ldots, n ; X_{i}$ é uma matriz conhecida $t_{i} \times p ; \beta$ é um vetor de parâmetros desconhecidos $p \times 1$; $e_{i}$ são independentemente distribuídos como $\mathrm{N}\left(0, \Sigma_{\mathrm{i}}\right)$, assumindo que cada elemento da matriz $\Sigma_{\mathrm{i}}$, para $\mathrm{i}=1, \ldots, \mathrm{n}$, são funções de q parâmetros de covariâncias desconhecidos contidos no vetor $\theta$.

Esse modelo com $\Sigma_{\mathbf{i}}$ permite examinar algumas, ou melhor, muitas alternativas de estruturas para $\Sigma_{i}$, sendo que cada estrutura de covariâncias tem sua importância e, em alguns casos, a sua formação apresenta uma parte fixa e outra aleatória, como no caso de modelos mistos, onde o modelo seria representado por

$$
y_{i}=X_{i} \beta+Z_{i} \gamma+e_{i}
$$

sendo $Z$ uma matriz de delineamento conhecida e $\gamma$ um vetor de parâmetros aleatórios desconhecidos. Por suposição teríamos que $\gamma$ e $\mathrm{e}_{i}$ são normalmente distribuídos com

$$
\mathrm{E}\left[\begin{array}{l}
\gamma \\
\mathrm{e}
\end{array}\right]=\left[\begin{array}{l}
0 \\
0
\end{array}\right] \text { e } \operatorname{Var}\left[\begin{array}{l}
\gamma \\
\mathrm{e}
\end{array}\right]=\sigma^{2}\left[\begin{array}{ll}
\mathrm{G} & 0 \\
0 & \mathrm{R}
\end{array}\right]
$$

então $y_{i} \cap N\left(X_{i} \beta, \sigma^{2}\left(Z_{i} G Z_{i}^{\prime}+R\right)\right)$ e seja $\Theta_{i}=\{g \in G$ e $r \in R\}$. 
Portanto, os vetores individuais $\mathrm{y}_{\mathrm{i}}, \mathrm{i}=1, \ldots, \mathrm{n}$, têm distribuições multivariadas independentes, ou seja,

$$
\mathrm{y}_{\mathrm{i}} \cap \mathrm{N}\left(\mathrm{X}_{\mathrm{i}} \beta, \Sigma_{\mathrm{i}}\right),
$$

onde $\sum_{\mathrm{i}}=\sigma^{2}\left(\mathrm{Z}_{\mathrm{i}} \mathrm{GZ} Z_{\mathrm{i}}^{\prime}+\mathrm{R}\right)$ e sua função de densidade é dada por

$$
f\left(y_{i}\right)=\frac{1}{(2 \pi)^{\frac{n}{2}}\left(\Sigma_{i} \mid\right)^{\frac{1}{2}}} \exp \left\{-\frac{1}{2}\left(y_{i}-X_{i} \beta\right)^{\prime}\left(\Sigma_{i}\right)^{-1}\left(y_{i}-X_{i} \beta\right)\right\} .
$$

O produto dessas funções é a função de verossimilhança

$$
L\left(\beta, \Sigma_{i}\right)=\prod_{i=1}^{n} \frac{1}{(2 \pi)^{\frac{n}{2}}\left(\left|\Sigma_{i}\right|\right)^{\frac{1}{2}}} \exp \left\{-\frac{1}{2}\left(y_{i}-X_{i} \beta\right)^{\prime}\left(\Sigma_{i}\right)^{-1}\left(y_{i}-X_{i} \beta\right)\right\} .
$$

Assim, com a transformação $-2 \ln \mathrm{L}$, tem-se

$$
\ell\left(\beta, \Sigma_{\mathrm{i}}\right)=-2 \ln \mathrm{L}=\sum_{\mathrm{i}=1}^{\mathrm{n}}\left[\mathrm{n} \ln (2 \pi)+\ln \left(\left|\Sigma_{\mathrm{i}}\right|\right)+\left(\mathrm{y}_{\mathrm{i}}-\mathrm{X}_{\mathrm{i}} \beta\right)^{\prime}\left(\Sigma_{\mathrm{i}}\right)^{-1}\left(\mathrm{y}_{\mathrm{i}}-\mathrm{X}_{\mathrm{i}} \beta\right)\right] .
$$

Portanto

$$
\ell=n \ln (2 \pi)+\sum_{\mathrm{i}=1}^{\mathrm{n}} \ln \left(\left|\Sigma_{\mathrm{i}}\right|\right)+\sum_{\mathrm{i}=1}^{\mathrm{n}}\left(\mathrm{y}_{\mathrm{i}}-\mathrm{X}_{\mathrm{i}} \beta\right)^{\prime}\left(\Sigma_{\mathrm{i}}\right)^{-1}\left(\mathrm{y}_{\mathrm{i}}-\mathrm{X}_{\mathrm{i}} \beta\right)
$$

Observe-se que, no modelo o efeito aleatório aparece através de $\Sigma_{\mathrm{i}}$. Podese escrever esse modelo também na forma do modelo componente de erro

$$
y_{i}=X_{i} \beta+e_{i}
$$

onde $e_{i}=Z_{i} \gamma+\varepsilon_{i}$ é o termo aleatório.

A estimativa de máxima verossimilhança é aquela que maximiza $\ell$. Isso pode ser feito derivando e igualando a zero as equações de máxima verossimilhança determinadas por:

1) $\frac{\partial \ell}{\partial \beta}$ e fazendo $\left.\frac{\partial \ell}{\partial \beta}\right|_{\beta=\hat{\beta} \in \Sigma_{i}=\hat{\Sigma}_{i}}=0$ 
2) $\frac{\partial \ell}{\partial \Sigma_{i}}$ e fazendo $\left.\frac{\partial \ell}{\partial \Sigma_{i}}\right|_{\beta=\hat{\beta} \in \Sigma_{i}=\hat{\Sigma}_{i}}=0$

onde $\hat{\beta}$ e $\hat{\Sigma}_{i}$ são os estimadores de máxima verossimilhança.

Desenvolvendo a equação $\frac{\partial \ell}{\partial \beta} \mid=-2 \mathrm{X}^{\prime} \Sigma^{-1}(\mathrm{y}-\mathrm{X} \beta)$ e igualando a zero temos que

$$
\hat{\beta}=\left(\sum_{i=1}^{n} X_{i}^{\prime} \hat{\Sigma}_{i}^{-1} X_{i}\right)^{-1}\left(\sum_{i=1}^{n} X_{i}^{\prime} \hat{\Sigma}_{i}^{-1} y_{i}\right)
$$

A esperança e a variância desse estimador podem ser obtidas facilmente:

$$
\begin{gathered}
E(\hat{\beta})=E\left[\left(X_{i}^{\prime} \hat{\Sigma}_{i}^{-1} X_{i}\right)^{-1}\left(X_{i}^{\prime} \hat{\Sigma}_{i}^{-1} y_{i}\right)\right] \\
E(\hat{\beta})=\left(X_{i}^{\prime} \hat{\Sigma}_{i}^{-1} X_{i}\right)^{-1}\left(X_{i}^{\prime} \hat{\Sigma}_{i}^{-1}\right) E\left(y_{i}\right) \\
E(\hat{\beta})=\left(X_{i}^{\prime} \hat{\Sigma}_{i}^{-1} X_{i}\right)^{-1} X_{i}^{\prime} \hat{\Sigma}_{i}^{-1} X_{i} \beta \\
E(\hat{\beta})=\beta
\end{gathered}
$$

e

$$
\begin{gathered}
\operatorname{Var}(\hat{\beta})=\operatorname{Var}\left(\left(\sum_{i=1}^{n} X_{i}^{\prime} \hat{\Sigma}_{i}^{-1} X_{i}\right)^{-1}\left(\sum_{i=1}^{n} X_{i}^{\prime} \hat{\Sigma}_{i}^{-1} y_{i}\right)\right) \\
\operatorname{Var}(\hat{\beta})=\left(\sum_{i=1}^{n} X_{i}^{\prime} \hat{\Sigma}_{i}^{-1} X_{i}\right)^{-1}\left(\sum_{i=1}^{n}\left(X_{i}^{\prime} \hat{\Sigma}_{i}^{-1} \operatorname{Var}\left(y_{i}\right) \hat{\Sigma}_{i}^{-1} X_{i}\right)\right)\left(\sum_{i=1}^{n} X_{i}^{\prime} \hat{\Sigma}_{i}^{-1} X_{i}\right)^{-1} \\
\operatorname{Var}(\hat{\beta})=\left(\sum_{i=1}^{n} X_{i}^{\prime} \hat{\Sigma}_{i}^{-1} X_{i}\right)^{-1}\left(\sum_{i=1}^{n}\left(X_{i}^{\prime} \hat{\Sigma}_{i}^{-1} X_{i}\right)\right)\left(\sum_{i=1}^{n} X_{i}^{\prime} \hat{\Sigma}_{i}^{-1} X_{i}\right)^{-1} \\
\operatorname{Var}(\hat{\beta})=\left(\sum_{i=1}^{n} X_{i}^{\prime} \hat{\Sigma}_{i}^{-1} X_{i}\right)^{-1}
\end{gathered}
$$


Desenvolvendo $\frac{\partial \ell}{\partial \Sigma_{\mathrm{i}}}$ e igualando a zero, têm-se as expressões para cada elemento da matriz de covariâncias de acordo com o modelo escolhido.

\subsubsection{Método da Máxima Verossimilhança Restrita (MVR)}

Pode-se observar que: a) o estimador de máxima verossimilhança não leva em consideração a perda dos graus de liberdade devidos à estimação dos efeitos fixos; b) os estimadores de máxima verossimilhança não coincidem, em geral, mesmo nos casos balanceados, com estimadores encontrados a partir de outros métodos, resultando em estimação viciada via máxima verossimilhança (Ciól, 1982).

Com o objetivo de melhorar essas deficiências foram desenvolvidos estimadores, chamados de máxima verossimilhança restrita, cuja idéia básica é calcular os estimadores de máxima verossimilhança eliminando os efeitos fixos.

No método da máxima verossimilhança restrita, a função de verossimilhança é fatorada em duas partes, sendo que uma delas é totalmente livre dos efeitos fixos.

A fatoração é realizada através de uma transformação linear nos dados originais.

\section{Considere-se o modelo}

$$
{ }_{n} y_{1}=X_{p} \beta_{1}+{ }_{n} e_{1}
$$

onde

y : é o vetor de observações;

$\mathrm{X}$ : é a matriz de delineamento conhecida com dimensão $\mathrm{n} x \mathrm{p}$ e posto $[\mathrm{X}]=\mathrm{p}$;

$\beta$ : é o vetor de parâmetros, e

e : é o vetor de erros. 
Sendo as seguintes suposições observadas

$$
\mathrm{y} \cap \mathrm{N}(\mathrm{X} \beta, \Sigma) \text { e e } \cap \mathrm{N}(0, \Sigma) \operatorname{com} \Sigma=\Sigma(\theta)
$$

e o vetor de observações apresentando a função densidade

$$
\mathrm{f}_{\mathrm{Y}}(\mathrm{y})=\frac{1}{(2 \pi)^{\frac{\mathrm{n}}{2}}(\Sigma)^{\frac{1}{2}}} \exp \left\{-\frac{1}{2}(\mathrm{y}-\mathrm{X \beta})^{\prime}(\Sigma)^{-1}(\mathrm{y}-\mathrm{X} \beta)\right\} .
$$

Para algum $\Sigma$ fixo, o estimador de $\beta$ de máxima verossimilhança dado por

(9) é

$$
\hat{\beta}=\left(X^{\prime} \hat{\Sigma}^{-1} X\right)^{-1} X^{\prime} \hat{\Sigma}^{-1} y={ }_{p} G_{n \text { n }} y_{1}
$$

$\operatorname{com} E(\hat{\beta})=\beta$ e $\operatorname{Var}(\hat{\beta})=\left(X^{\prime} \Sigma^{-1} X\right)^{-1}$, então

$$
\hat{\beta} \cap N\left(\beta,\left(X^{\prime} \Sigma^{-1} X\right)^{-1}\right)
$$

e apresenta a função densidade

$$
f_{\hat{\beta}}(\hat{\beta})=\frac{1}{(2 \pi)^{\frac{p}{2}}\left(X^{\prime} \Sigma^{-1} X\right)^{\frac{1}{2}}} \exp \left\{-\frac{1}{2}(\hat{\beta}-\beta)\left(X^{\prime} \Sigma^{-1} X\right)(\hat{\beta}-\beta)\right\} .
$$

Seja

$$
\mathrm{P}=\mathrm{I}-\mathrm{X}\left(\mathrm{X}^{\prime} \mathrm{X}\right)^{-1} \mathrm{X}^{\prime}
$$

onde P é o projetor ortogonal de ê no espaço coluna de y. Então Py $=\hat{\mathrm{e}}_{\mathrm{oLs}}$, ê oLs é o resíduo de mínimos quadrados ordinários. $O$ posto de $P$ é n-p, isto é, $P$ tem n-p linhas linearmente independentes.

Seja ${ }_{n} A_{n-p}$ tal que $A A^{\prime}=P$ e $A^{\prime} A=I$ o posto de $A$ também é $n-p$. As colunas de A produzem um conjunto de coeficientes para n-p contrastes de erro linearmente independentes.

Pode-se então tomar colunas de $\mathrm{P}$ ou alguma outra matriz de posto completo com $\mathrm{E}\left(\mathrm{y}^{*}\right)=0$, onde $\mathrm{y}^{*}=\mathrm{My}$, e $\mathrm{M}$ é a matriz considerada.

Escolhe-se A definida como $\mathrm{AA}^{2}=\mathrm{P}$ e A'A $=\mathrm{I}$, como matriz $\mathrm{M}$.

Considere-se, agora, um "novo" conjunto de dados:

$$
\begin{gathered}
R=A^{\prime} y \\
R \cap N\left(0, A^{\prime} \sum A\right) .
\end{gathered}
$$


Assim,

$$
\begin{gathered}
E(R)=E\left(A^{\prime} y\right)=A^{\prime} E(y)=I^{\prime} E(y)=A^{\prime} A A^{\prime} E(y)=A^{\prime} P X \beta \\
E(R)=A^{\prime}\left[I-X\left(X^{\prime} X\right)^{-1} X^{\prime}\right] X \beta=A^{\prime}\left[X-X\left(X^{\prime} X\right)^{-1} X^{\prime} X\right] \beta \\
E(R)=A^{\prime}[X-X] \beta=0
\end{gathered}
$$

e

$$
\operatorname{Var}(\mathrm{R})=\operatorname{Var}\left(\mathrm{A}^{\prime} \mathrm{y}\right)=\mathrm{A}^{\prime} \operatorname{Var}(\mathrm{y}) \mathrm{A}=\mathrm{A}^{\prime} \sum \mathrm{A}
$$

Note que $\mathrm{R}$ é uma combinação linear de $\mathrm{y}$, e que $\mathrm{R}$ tem todas as informações sobre $\Sigma$, e tem as dimensões $(n-p) \times 1$ e $\hat{\beta}$ é $p \times 1$.

É possivel mostrar que $R$ e $\hat{\beta}$ são independentes, porque $\operatorname{Cov}(R, \hat{\beta})=0$, sob normalidade, garante a independência.

$$
\begin{gathered}
\operatorname{Cov}(R, \hat{\beta})=E\left\{[R-E(R)][\hat{\beta}-E(\hat{\beta})]^{\prime}\right\}=E\left[R(\hat{\beta}-\beta)^{\prime}\right] \\
\operatorname{Cov}(R, \hat{\beta})=E\left[A^{\prime} y\left(y^{\prime} G^{\prime}-\beta^{\prime}\right]=E\left[A^{\prime} y y^{\prime} G^{\prime}-A^{\prime} y \beta^{\prime}\right]\right. \\
\operatorname{Cov}(R, \hat{\beta})=A^{\prime} E\left(y y^{\prime}\right) G^{\prime}-A^{\prime} E(y) \beta^{\prime} \\
\operatorname{Cov}(R, \hat{\beta})=A^{\prime}\left[\operatorname{Var}(y)+E(y)+E\left(y^{\prime}\right)\right] G^{\prime}-A^{\prime} X \beta \beta^{\prime} \\
\operatorname{Cov}(R, \hat{\beta})=A^{\prime}\left[\Sigma+X \beta \beta^{\prime} X^{\prime}\right] G^{\prime}-A^{\prime} X \beta \beta^{\prime} \\
\operatorname{Cov}(R, \hat{\beta})=A^{\prime}\left[\Sigma \Sigma^{-1} X\left(X^{\prime} \Sigma^{-1} X\right)^{-1}+X \beta \beta^{\prime} X^{\prime} \Sigma^{-1} X\left(X^{\prime} \Sigma^{-1} X\right)^{-1}\right]-A^{\prime} X \beta \beta^{\prime} \\
\operatorname{Cov}(R, \hat{\beta})=A^{\prime} X\left(X^{\prime} \Sigma^{-1} X\right)^{-1}+A^{\prime} X \beta \beta^{\prime}-A^{\prime} X \beta \beta^{\prime} \\
\operatorname{Cov}(R, \hat{\beta})=I A^{\prime} X\left(X^{\prime} \Sigma^{-1} X\right)^{-1}=A^{\prime} A A^{\prime} X\left(X^{\prime} \Sigma^{-1} X\right)^{-1} \\
\operatorname{Cov}(R, \hat{\beta})=A\left(P X\left(X^{\prime} \Sigma^{-1} X\right)^{-1}=A^{\prime}\left[I-X\left(X^{\prime} X\right)^{-1} X^{\prime}\right] X\left(X^{\prime} \Sigma^{-1} X\right)^{-1}\right. \\
\operatorname{Cov}(R, \hat{\beta})=A^{\prime}\left[X-X\left(X^{\prime} X\right)^{-1} X^{\prime} X\right]\left(X^{\prime} \Sigma^{-1} X\right)^{-1} \\
\operatorname{Cov}(R, \hat{\beta})=A^{\prime}[0]\left(X^{\prime} \Sigma^{-1} X\right)^{-1}=0
\end{gathered}
$$

Então, temos: 


$$
\left\{\begin{array}{c}
\hat{\beta}=\text { Gy e } R=A^{\prime} y,{ }_{p} G_{n} \text { e }{ }_{n-p} A_{n}^{\prime} \\
\hat{\beta} \text { e } R \text { são independentes } \\
\operatorname{posto}(G)=p \text { e posto }(A)=n-p
\end{array}\right.
$$

Com isso é possível construir uma matriz de posto completo $\mathrm{n} \times \mathbf{n}$

$$
\left[\begin{array}{l}
A^{\prime} \\
G
\end{array}\right] \text { tal que }\left[\begin{array}{l}
A^{\prime} \\
G
\end{array}\right] y=\left[\begin{array}{c}
R \\
\hat{\beta}
\end{array}\right] \text {. }
$$

A distribuição de $\left[\begin{array}{c}R \\ \hat{\beta}\end{array}\right]$ é normal (combinação linear de y) e, devido à independência entre $\mathrm{R}$ e $\hat{\boldsymbol{\beta}}$, a função densidade pode ser escrita como:

$$
f_{\left[\begin{array}{l}
R \\
\hat{\beta}
\end{array}\right]}([r \hat{\beta}])=f_{R}(r) f_{\hat{\beta}}(\hat{\beta})
$$

A expressão $\left[\begin{array}{l}A^{\prime} \\ G\end{array}\right] y=\left[\begin{array}{c}R \\ \hat{\beta}\end{array}\right]$ é uma transformação de um vetor aleatório.

Então, considerando $y=\left[\begin{array}{l}A^{\prime} \\ G\end{array}\right]^{-1}\left[\begin{array}{l}R \\ \hat{\beta}\end{array}\right]$

$$
f_{Y}(y)=|J| f_{[R]}(y)
$$

Assim,

$$
f_{Y}(y)=|J| f_{R}(y) f_{\hat{\beta}}(y)
$$

onde $|J|=\left|X^{\prime} X\right|^{-\frac{1}{2}}$

Queremos a função densidade de R, expressa em termos de y.

$$
f_{R}(y)=|J|^{-1}\left[\frac{f_{Y}(y)}{f_{\hat{\beta}}(y)}\right] \text {. }
$$

Então 


$$
\begin{aligned}
& f_{R}(y)=\left|X^{\prime} X\right|^{\frac{1}{2}} \frac{(2 \pi)^{-\frac{n}{2}}|\Sigma|^{-\frac{1}{2}} \exp \left\{-\frac{1}{2}(y-X \beta)^{\prime} \Sigma^{-1}(y-X \beta)\right\}}{(2 \pi)^{-\frac{p}{2}}\left|X^{\prime} \Sigma^{-1} X\right|^{\frac{1}{2}} \exp \left\{-\frac{1}{2}(\hat{\beta}-\beta)^{\prime}\left(X^{\prime} \Sigma^{-1} X\right)(\hat{\beta}-\beta)\right\}} \\
& f_{R}(y)=\left|X^{\prime} X\right|^{\frac{1}{2}}(2 \pi)^{-\frac{n-p}{2}}|\Sigma|^{-\frac{1}{2}}\left|X^{\prime} \Sigma^{-1} X\right|^{-\frac{1}{2}} * \\
& * \exp \left\{-\frac{1}{2}\left[(y-X \beta)^{\prime} \Sigma^{-1}(y-X \beta)+(\beta-\hat{\beta})^{\prime}\left(X^{\prime} \Sigma^{-1} X\right)(\beta-\hat{\beta})\right]\right\}
\end{aligned}
$$

Mas, o termo dentro do exponencial pode ser substituído, segundo Harville (1974), pelo seguinte:

$$
f_{R}(y)=\left|X^{\prime} X\right|^{\frac{1}{2}}(2 \pi)^{-\frac{n-p}{2}}|\Sigma|^{-\frac{1}{2}}\left|X^{\prime} \Sigma^{-1} X\right|^{-\frac{1}{2}} \exp \left\{-\frac{1}{2}(y-X \hat{\beta})^{\prime} \Sigma^{-1}(y-X \hat{\beta})\right\},
$$

que é uma verossimilhança associada com $\mathrm{R}$. Como $\mathrm{R}$ é residuo de mínimos quadrados ordinários, essa verossimilhança é chamada de máxima verossimilhança restrita (Restrita no sentido que se refere somente a $\Sigma$ e não a $\beta$ ).

A prova da passagem dos termos dentro do exponencial é apresentada a seguir:

$$
\begin{gathered}
\left\{-\frac{1}{2}\left[(y-X \beta)^{\prime} \Sigma^{-1}(y-X \beta)+(\beta-\hat{\beta})^{\prime}\left(X^{\prime} \Sigma^{-1} X\right)(\beta-\hat{\beta})\right]\right\} \\
\left\{-\frac{1}{2}\left[(y-X \beta)^{\prime} \Sigma^{-1}(y-X \beta)+(X \beta-X \hat{\beta})^{\prime} \Sigma^{-1}(X \beta-X \hat{\beta})\right]\right\} \\
\left\{-\frac{1}{2}\left[(y-X \beta+X \beta-X \hat{\beta})^{\prime} \Sigma^{-1}(y-X \beta+X \beta-X \hat{\beta})\right]\right\} \\
\left\{-\frac{1}{2}\left[(y-X \hat{\beta})^{\prime} \Sigma^{-1}(y-X \hat{\beta})\right]\right\} .
\end{gathered}
$$

Dada a máxima verossimilhança na função anterior, para um conjunto de dados, somente $\Sigma$ é a quantidade desconhecida. Mas $\Sigma$ pode ser definida com o vetor $\theta$, então $\Sigma=\Sigma(\theta)$. 


\section{MATERIAL E MÉTODOS}

Os dados utilizados para análise foram provenientes de um experimento realizado no período de julho de 1997 a outubro de 1998, conduzido por Shoey Kanashiro, no Departamento de Fitotecnia, da Escola Superior de Agricultura "Luiz de Queiroz" - Universidade de São Paulo, Piracicaba, SP como parte de sua dissertação de mestrado.

O delineamento experimental foi aleatorizado em blocos, com 4 blocos, contendo 8 plantas por parcela. O material experimental consistiu de 15 substratos (considerados tratamentos) utilizados para o cultivo de Bromélias (Aechmea fasciata) em vaso. Esses 15 substratos consistiam da combinação de algum material que pudesse ser utilizado na substituição de xaxim sempre na companhia de turfa e perlita, em três diferentes proporções desses materiais $(2: 7: 1),(5: 4: 1)$ e (8:1:1). Neste trabalho serão utilizados os dados referentes à proporção ( $5: 4: 1$ ), tendo-se os seguintes tratamentos:

T1 (5:4:1): Casca de Pinus + turfa + perlita

T2 (5:4:1): Casca de Eucaliptos + turfa + perlita

T3 (5:4:1): Coxim + turfa + perlita

T4 (5:4:1): Fibra de coco + turfa + perlita

T5 (5:4:1): Xaxim + turfa + perlita

Será utilizada a variável Número Médio de Folhas, para a qual foram realizadas 6 medidas no decorrer do tempo:

D5: medida realizada aos 5 dias após o plantio;

D173: medida realizada aos 173 dias após o plantio;

D229: medida realizada aos 229 dias após o plantio;

D285: medida realizada aos 285 dias após o plantio; 
D341: medida realizada aos 341 dias após o plantio;

D435: medida realizada aos 435 dias após o plantio.

Os dados referentes a essa variável são apresentados na Tabela 2 a seguir.

Tabela 2. Dados obtidos do experimento com Bromélias em função dos tratamentos e das medidas realizadas ao longo do tempo para a variável Número Médio de Folhas.

\begin{tabular}{ccccccccc}
\hline Parcela & Trat. & Blocos & D5 & D173 & D229 & D285 & D341 & D435 \\
\hline 01 & T1 & 1 & 6,50 & 9,00 & 11,25 & 14,25 & 16,13 & 17,50 \\
02 & T1 & 2 & 6,25 & 9,25 & 11,63 & 14,29 & 17,00 & 17,67 \\
03 & T1 & 3 & 6,38 & 8,75 & 11,13 & 13,38 & 15,50 & 16,00 \\
04 & T1 & 4 & 7,13 & 9,00 & 12,00 & 14,63 & 16,63 & 17,83 \\
05 & T2 & 1 & 6,75 & 8,13 & 10,75 & 12,00 & 14,57 & 15,17 \\
06 & T2 & 2 & 6,13 & 7,13 & 9,88 & 12,63 & 14,50 & 15,33 \\
07 & T2 & 3 & 6,38 & 7,38 & 9,63 & 11,88 & 13,88 & 15,00 \\
08 & T2 & 4 & 6,88 & 7,38 & 10,75 & 13,00 & 14,75 & 16,00 \\
09 & T3 & 1 & 6,50 & 8,00 & 11,00 & 12,57 & 14,29 & 15,29 \\
10 & T3 & 2 & 6,50 & 8,25 & 10,63 & 13,13 & 15,00 & 15,83 \\
11 & T3 & 3 & 6,38 & 7,50 & 10,25 & 12,88 & 14,63 & 15,67 \\
12 & T3 & 4 & 6,88 & 8,38 & 11,50 & 14,38 & 15,75 & 16,67 \\
13 & T4 & 1 & 6,38 & 7,75 & 10,50 & 12,88 & 14,38 & 15,83 \\
14 & T4 & 2 & 6,25 & 8,50 & 10,00 & 13,13 & 15,88 & 16,17 \\
15 & T4 & 3 & 6,38 & 7,88 & 10,25 & 12,50 & 14,13 & 14,83 \\
16 & T4 & 4 & 6,88 & 8,63 & 11,38 & 13,13 & 14,75 & 16,17 \\
17 & T5 & 1 & 6,75 & 9,88 & 13,25 & 13,50 & 15,33 & 16,33 \\
18 & T5 & 2 & 6,50 & 10,25 & 12,88 & 13,83 & 16,83 & 17,33 \\
19 & T5 & 3 & 6,75 & 8,63 & 11,50 & 13,50 & 15,38 & 16,00 \\
20 & T5 & 4 & 6,88 & 8,75 & 11,75 & 13,88 & 16,13 & 17,00 \\
\hline
\end{tabular}

Com o auxílio desses dados serão discutidos modos de se analisarem dados de medidas repetidas através de dois procedimentos do SAS, o proc GLM e o proc MIXED, sobre os quais será realizada, a seguir, uma descrição, bem como comparação.

Para dados de medidas repetidas o proc GLM utiliza uma estrutura tradicional para obter os resultados, tanto do modelo univariado como do multivariado. Mas, quando se trata de modelos mistos, o proc MIXED é o mais indicado.

Primeiramente, o proc GLM requer que os dados sejam balanceados, não utilizando dados de indivíduos que tenham algum valor perdido, por utilizar o método dos momentos, que precisa de dados completos para estimar os efeitos. Depois que se 
identificam os indivíduos com dados completos, seleciona-se para analisar um modelo de médias, em termos de efeitos fixos da parcela e da subparcela, entre e intra-indivíduos, respectivamente.

O fator da parcela (entre indivíduos) é aquele cujos níveis permanecem constantes, ao passo que o fator da subparcela e interação parcela $\times$ subparcela (intraindivíduos) varia.

No caso do experimento utilizado, os efeitos da parcela são os Tratamentos, os efeitos da subparcela são os Dias e a interação Dias $\times$ Tratamentos. No caso do proc GLM é necessário especificar esses efeitos separadamente.

O proc GLM requer que seja indicada uma transformação (tipo de contraste) para os dados de medidas repetidas, já que um conjunto de contrastes pode ser usado para analisar tendências sobre o fator de medidas repetidas, e realizar comparações entre os níveis desse mesmo fator. Os dados originais de cada indivíduo são transformados em um novo conjunto de dados, obtidos através de um conjunto de $\mathrm{t}-1$ contrastes, onde $t$ é o número de medidas repetidas. Essas transformações (contrastes) são utilizadas na tentativa de amenizar a influência de algumas estruturas de covariâncias, na análise univariada de medidas repetidas, que podem invalidar os resultados dos testes. As transformações disponíveis são:

a) Contraste: gera contrastes entre os niveis do fator tempo, podendo-se optar por um nível de referência, caso não seja especificado, o contraste é construido utilizando o último nível;

b) Polinomial: gera contrastes polinomiais ortogonais;

c) Helmert: gera contrastes entre cada nível do fator tempo e a média dos níveis subseqüentes;

d) Médias: gera contrastes entre os níveis do fator tempo e a média de todos os outros níveis do fator tempo;

e) Perfil: gera contrastes entre os níveis adjacentes do fator tempo.

O proc GLM apresenta um teste padrão para os efeitos fixos (parcela). 
Com relação aos efeitos da subparcela, existem duas opções de testes: o univariado e o multivariado. Os testes univariados são válidos quando a condição de H-F para a matriz de covariâncias é satisfeita, podendo esta suposição ser verificada pelo teste de esfericidade, já implementado no proc GLM. Quando esse teste de esfericidade é significativo, pode-se optar pela correção do número de graus de liberdade, para os efeitos da subparcela, e assim realizar a análise univariada, ou ainda pelos testes multivariados: Lambda de Wilks, Traço de Pillai, Traço de Hotelling-Lawley e Roy. Esses testes são todos baseados em uma matriz de covariâncias sem estrutura.

O esquema que o proc GLM utiliza para a análise de medidas repetidas pode ser observado na Figura 4 a seguir: 


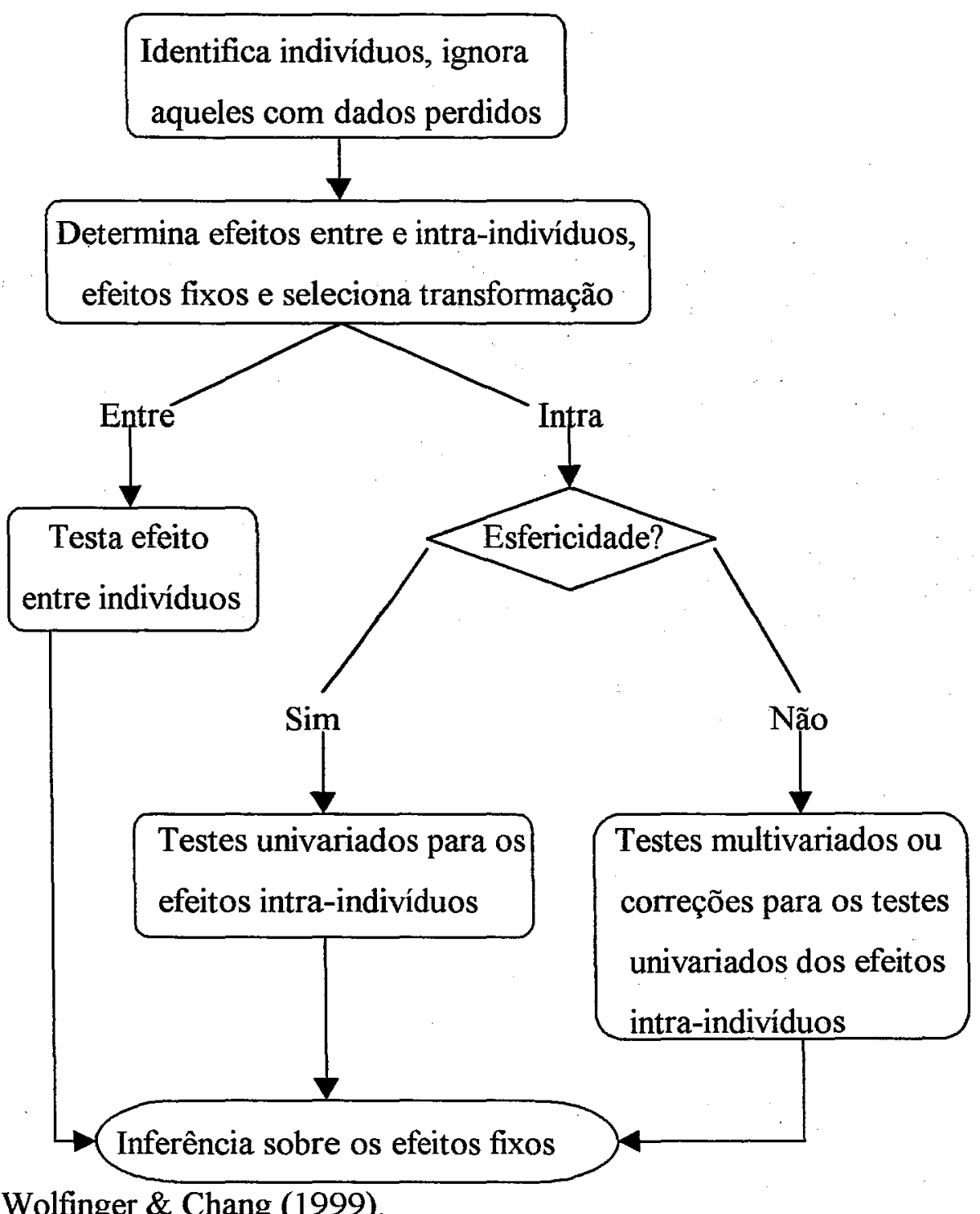

Fonte: Wolfinger \& Chang (1999).

Figura 4. Esquema da análise de medidas repetidas no proc GLM do SAS.

O proc MIXED, ao contrário do proc GLM, permite a inclusão de indivíduos que tenham alguma observação perdida, isso, porque utiliza os métodos da máxima verossimilhança, máxima verossimilhança restrita e MIVIQ0.

Não há necessidade de fazer uma distinção inicial dos efeitos entre e intraindivíduos, simplesmente determina-se o modelo de médias, sem a necessidade de trabalhar com uma transformação para os dados. Mas, é preciso especificar a estrutura da matriz de covariâncias que melhor represente os dados, pois os testes para os efeitos 
fixos são baseados nessa estrutura da matriz de covariâncias, e caso seja mal especificada produzirá resultados inválidos.

O processo de seleção da matriz de covariâncias pode ser feito utilizandose o AIC (critério de informação de Akaike), já implementado, ou então, construindo-se um teste de razão de verossimilhança, entre estruturas de covariâncias duas a duas, mas com a condição de que essas estruturas sejam casos especiais umas das outras (dentro do par).

Da mesma forma como mostrado pela Figura 4, para o proc GLM, o esquema de análise de medidas repetidas pelo proc MIXED é apresentado pela Figura 5:

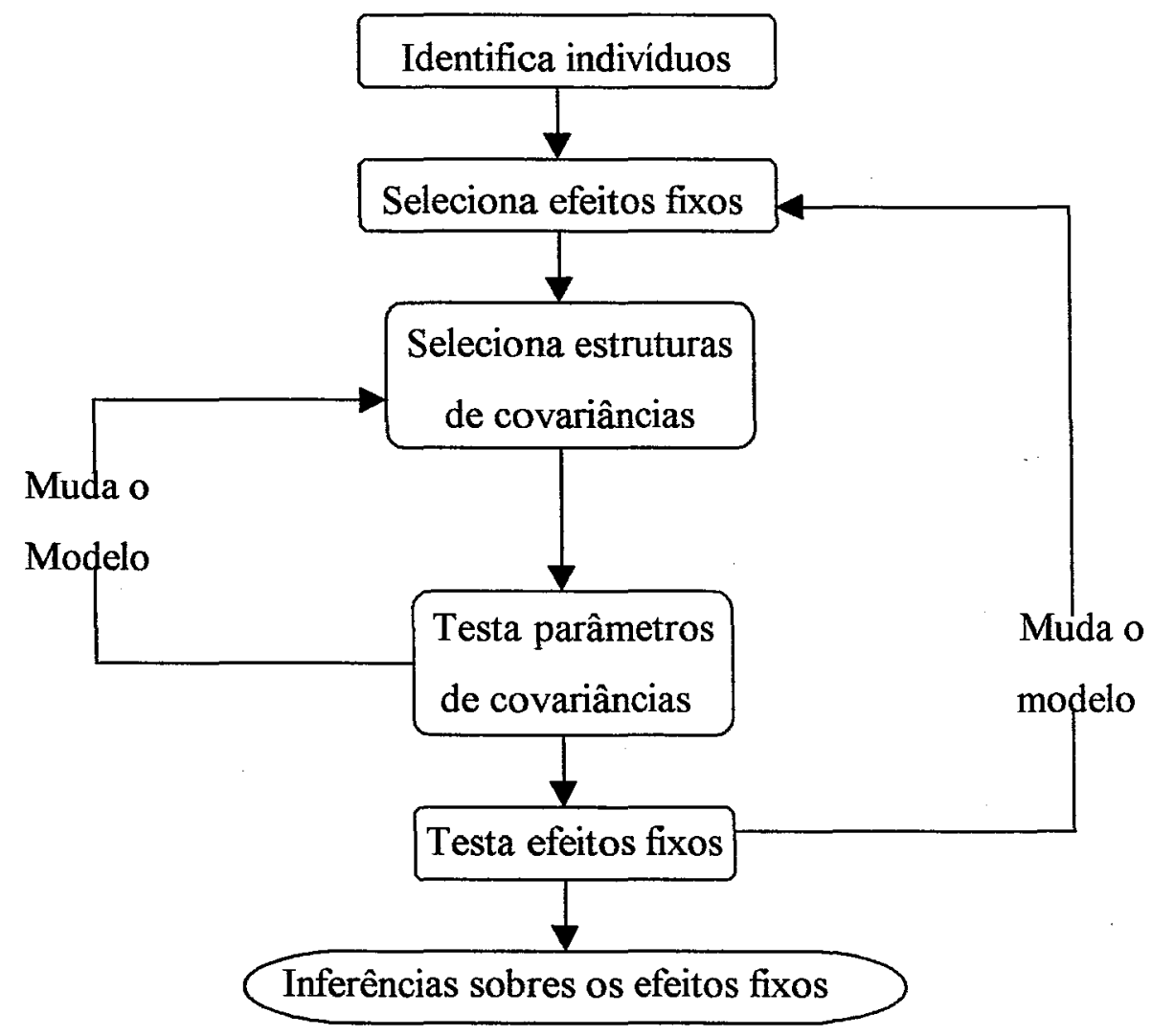

Fonte: Wolfinger \& Chang (1999).

Figura 5. Esquema da análise de medidas repetidas no proc MIXED do SAS.

Um resumo das comparações dos dois procedimentos é apresentado na Tabela 3. 
Tabela 3. Resumo de comparações de procedimentos para análise de medidas repetidas.

$\frac{\text { PROC GLM }}{\text { Requer dados balanceados; ignora indivíduos com }}$ Permite dados com observações perdidas.
observações perdidas.

Lida com efeitos entre e intra-individuos Lida com efeitos entre e intra-indivíduos diferentemente com respeito à sintaxe e testes. similarmente.

Requer uma transformação ortogonal para variável Analisa os dados na sua forma original. de medidas repetidas.

Assume um modelo ANOVA completo (média de Permite uma ANOVA completa e / ou um modelo caselas) para o efeito intra-indivíduos. de médias reduzido para o efeito intra-indivíduos.

Assume que covariáveis são constantes dentro de Permite variação de covariáveis dentro de um um individuo. indivíduo.

Forma automaticamente um teste de esfericidade Pode produzir teste de esfericidade resultante de com a opção PRINTE. ambos os procedimentos TYPE $=\mathrm{UN}$ e TYPE $=\mathrm{HF}$ ou usando TYPE $=\mathrm{UN}$ em dados transformados.

Assume qualquer uma das duas estruturas da Permite uma ampla variedade de estruturas de matriz de covariâncias, do tipo $\mathrm{H}$ ou sem estrutura. covariâncias para o efeito intra-indivíduos.

Estima parâmetros de covariâncias usando o Estima parâmetros de covariâncias usando máxima método dos momentos. verossimilhança restrita, máxima verossimilhança, e MIVQUE0.

É computacionalmente rápido e fácil, e mostra Pode ser computacionalmente intensivo e requer todos os testes significantes em um procedimento. diferentes procedimentos, para diferentes estruturas de covariâncias.

Calcula estatísticas $\mathrm{F}$ que são razões de quadrados Calcula estatísticas $\mathrm{F}$ que são formas quadráticas médios. do tipo-Wald.

Calcula critérios G-G e H-F para testes univariados Calcula somente critérios para testes univariados para medidas repetidas,. para medidas repetidas (usando TYPE $=\mathrm{CS}$ ou TYPE=HF).

Calcula 4 testes multivariados para medidas Calcula um F tipo-Wald (usando TYPE=UN) e repetidas: Lambda de Wilks, Traço de Pillai, Traço duas versões do Traço de Hotelling-Lawley.

de Hotelling-Lawley e Roy.

Calcula LSMEANS somente para cada variável Calcula LSMEANS os quais são médias sobre separadamente. medidas repetidas e cujos erros padrão refletem a estrutura de covariâncias adequada.

Fonte: Wolfinger \& Chang (1999). 
O modelo univariado num esquema de parcelas subdivididas com medidas repetidas no tempo, é a técnica mais utilizada com relação aos modelos multivariado e misto. Mas, como visto anteriormente, a técnica univariada requer certa condição para a matriz de covariâncias. Pelo fato de ser muito utilizada na prática, optou-se por verificar, através de simulações, a acurácia da análise estatística quando de seu emprego, através da observação de casos em que a matriz de covariâncias atende, ou não, à condição de esfericidade, pois, segundo Shimizu (1975) e Dachs (1988) simulação é o processo de imitar o comportamento de um sistema real para estudar seu comportamento sob diferentes condições.

As simulações foram realizadas com o intuito de verificar o que ocorre com os testes $\mathrm{F}$ e níveis de significância dos mesmos, em nível de subparcela, quando a condição de circularidade é satisfeita, ou não, e também quando ocorre desbalanceamento. Os dados serão simulados observando-se o mesmo delineamento experimental utilizado por Kanashiro (1999).

Segundo Dias (1996) para se gerarem variáveis aleatórias multivariadas é necessário levar em conta a estrutura de correlação multivariada, que faz com que várias variáveis sejam geradas coletivamente, tornando o processo de simulação mais complexo do que para o caso univariado.

O método utilizado para a geração dos dados foi o das distribuições condicionais. Já que o mesmo reduz o problema da geração de um vetor t-dimensional em uma série de $t$ gerações univariadas (Johnson, 1987).

Uma distribuição t-dimensional pode ser representada como um produto de $t$ distribuições condicionais.

A geração de variáveis aleatórias de uma distribuição multivariada pode ser realizada pela geração, em seqüência, de observações de cada uma das distribuições condicionais, através da densidade conjunta do vetor aleatório $\mathrm{X}$, que pode ser fatorado da seguinte forma:

$$
f\left(x_{1}, x_{2}, \ldots, x_{t}\right)=f_{1}\left(x_{1}\right) f_{2}\left(x_{2} / x_{1}\right) \ldots f_{t}\left(x_{t} / x_{1}, \ldots, x_{t-1}\right)
$$

Os passos desse método podem ser assim resumidos: 
1. Gerar $X_{1}=x_{1}$ de uma distribuição marginal de $X_{1}$.

2. Gerar $X_{2}=x_{2}$ de uma distribuição condicional de $X_{2}$ dado $X_{1}=x_{1}$.

3. Gerar $X_{3}=x_{3}$ de uma distribuição condicional de $X_{3}$ dado $X_{1}=x_{1}$ e $\mathrm{X}_{2}=\mathrm{X}_{2}$.

4. E assim por diante p passos.

Segundo Boswell (1993), os principais obstáculos para a implementação desse método são: determinação da distribuição condicional, e identificação de uma técnica de geração univariada adequada para cada uma das distribuições condicionais.

Foram simulados dados através do "software" SAS, utilizando-se as distribuições normal e normal contaminada (que é a soma de duas normais ponderadas).

Para a geração de variáveis aleatórias normais multivariadas, o método das distribuições condicionais supõem que $X$ segue uma distribuição normal multivariada com vetor de médias $\mu=\left(\mu_{1}, \ldots, \mu_{\mathrm{t}}\right)$ e matriz de covariâncias $\sum=\left(\sigma_{\mathrm{ij}}\right)$, positiva definida. Para $\mathrm{i}=1, \ldots, \mathrm{t}$, define-se $\mathrm{X}_{(\mathrm{i})}=\left(\mathrm{X}_{1}, \ldots, \mathrm{X}_{\mathrm{i}}\right)^{\prime}$ como vetor da primeira componente de X. Assim, de $\mathrm{X}$ simulado através da distribuição normal tem-se que

$$
X \cap N_{t}(\mu, \Sigma)
$$

e dados simulados através da distribuição normal contaminada

$$
\mathrm{X}=\alpha \mathrm{N}_{\mathrm{t}}\left(\mu_{1}, \Sigma_{1}\right)+(1-\alpha) \mathrm{N}_{\mathrm{t}}\left(\mu_{2}, \Sigma_{2}\right)
$$

onde $\alpha$ e (1- $\alpha)$ são as ponderações utilizadas.

Para os casos simulados com a normal contaminada as ponderações utilizadas serão $\alpha=0,05,0,10,0,15$ e 0,20 .

Para a simulação foi considerado o modelo matricial:

$$
Y_{(g b \times t)}=X_{(g b \times(g+b+1))} B_{((g+b+1) \times t)}+\Psi_{(g b \times t)}
$$

onde g é o número de tratamentos, b é o número de blocos e $\mathrm{t}$ é o número de medidas repetidas, e os termos do modelo são definidos em (4).

Malheiros (1999) simulou dados em um esquema de aleatorização em blocos, com parcelas subdivididas com medidas repetidas, provenientes de uma 
distribuição normal, considerando, porém, variâncias iguais para as estruturas de covariâncias utilizadas e efeitos pouco significativos. Neste trabalho serão simulados dados com a distribuição normal e a distribuição normal contaminada, levando-se em conta estruturas de matrizes de covariâncias com maior variabilidade do que aquelas utilizadas por Malheiros (1999). Além disso considerar-se-ão duas situações para os efeitos dos fatores, ou seja, efeitos nulos e efeitos não nulos.

Através dos resultados obtidos com simulações utilizando efeitos nulos, verificar-se-á se os níveis mínimos de significância dos testes $\mathrm{F}$, associados às hipóteșes para a subparcela e interação parcela ×subparcela, apresentam distribuição uniforme $(0,1)$.

De acordo com Malheiros (1999), esses níveis mínimos de significância podem ser distribuídos em classes de frequências de amplitude 0,05 , no intervalo $(0,1)$, tendo-se dessa forma 20 classes de frequências. A acurácia dos testes $\mathrm{F}$ na análise univariada será avaliada através de um teste Qui-quadrado para testar a hipótese de aderência da distribuição dos níveis mínimos de significância à distribuição uniforme. A acurácia dos testes $\mathrm{F}$ será melhor quanto mais os níveis mínimos de significância se aproximam da distribuição uniforme $(0,1)$, pois, segundo Mood et al. (1974) caso as exigências do teste $F$, para a análise univariada, sejam satisfeitas sob hipótese nula, os níveis mínimos de significância terão distribuição uniforme.

Com os resultados obtidos das simulações utilizando efeitos não nulos, a distribuição dos níveis mínimos de significância poderá auxiliar na detecção de situações em que os testes são mais sensíveis em indicar a existência desses efeitos (Malheiros, 1999). 


\section{RESULTADOS E DISCUSSÕES}

\subsection{Procedimentos do GLM e MIXED}

A estrutura dos dados para a análise de medidas repetidas é diferente para os dois procedimentos. Por esse motivo, faz-se necessária a construção de dois conjuntos de dados, um vetorizado na forma univariada e outro na forma multivariada. Assim, o proc GLM utiliza o conjunto de dados do arquivo "Multiv" para a análise multivariada, e o conjunto de dados do arquivo "Univ" para análise univariada. Já o proc MIXED utiliza apenas o conjunto de dados do arquivo "Univ" para as análises dos modelos univariado, multivariado e misto. Os conjuntos de dados dos arquivos "Multiv" e "Univ" podem ser obtidos da seguinte forma:

data multiv (keep=parcela trat blocos d5 d173 d229 d285 d341 d435)
univ (keep=parcela trat blocos dias $\mathrm{nf}$ );
input parcela trat blocos d5 d173 d229 d285 d341 d435;
output multiv;
nf=d5; dias=5; output univ;
nf=d173; dias=173; output univ;
nf=d229; dias=229; output univ;
nf=d285; dias=285; output univ;
nf=d341; dias=341; output univ;
nf=d435; dias=435; output univ;
cards;
nf


Pode-se obter a análise univariada utilizando-se procedimentos tanto do proc GLM como do proc MIXED.

Com a análise univariada através do proc GLM, usando um modelo de parcelas subdivididas com medidas repetidas no tempo, os testes envolvendo o fator intra-individuos podem não ser válidos por não atenderem a condição de H-F. Porém, o teste para o fator entre indivíduos é válido quando se especifica o termo de erro corretamente. O programa deve, então, seguir os seguintes passos:

proc glm data=univ;

class trat blocos dias parcela;

model $n f=b l o c o s$ trat blocos*trat dias dias*trat;

run;

test $h=$ trat $e=b l o c o s * t r a t ;$

onde a opção TEST especifica que o fator tratamentos deve ser testado com o termo da interação blocosxtratamentos (erro da parcela).

Os resultados dessa forma obtidos são apresentados na Tabela 4, a seguir.

Tabela 4. Resultados da análise univariada usando o proc GLM.

\begin{tabular}{lrrrrr}
\hline Variável Dependente: & Número & Médio de Folhas & & \\
\hline Causas de Variação & G.L. & S.Q. & Q.M. & F & Pr $>$ F \\
\hline Modelo & 44 & 1515,68690333 & 34,44742962 & 220,53 & 0,0001 \\
Residuo & 75 & 11,71509583 & 0,15620128 & \\
\hline Total Corrigido & 119 & 1527,40199917 & & \\
\hline BLOCOS & 3 & 10,95070917 & 3,65023639 & 23,37 & 0,0001 \\
TRAT & 4 & 37,45778667 & 9,36444667 & 27,56 & 0,0001 \\
TRAT*BLOCoS & 12 & 4,07742000 & 0,33978500 & & \\
DIAS & 5 & 1453,11191417 & 290,62238283 & 1860,56 & 0,0001 \\
TRAT*DIAS & 20 & 10,08907333 & 0,50445367 & 3,23 & 0,0001 \\
\hline \multicolumn{7}{c}{ C.V. 3,348899 } \\
\hline
\end{tabular}

Esses resultados, univariados, também podem ser obtidos usando-se a análise multivariada do proc GLM, que fornece o teste de esfericidade e os testes F válidos para os fatores intra-indivíduos, com as devidas correções para o número de graus de liberdade. O programa, então, é estruturado da seguinte forma: 


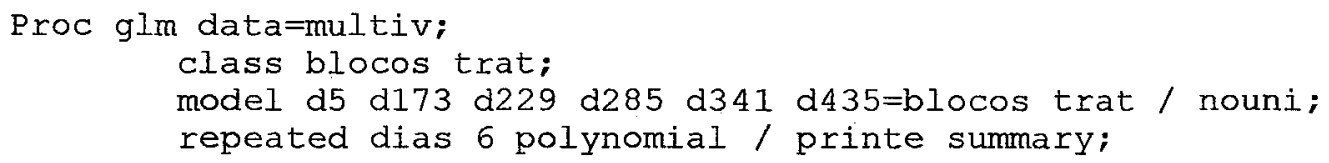

O comando MODEL ajusta uma análise multivariada de variância, sendo listados nesse comando somente os fatores entre indivíduos, nesse caso blocos e tratamentos. A opção NOUNI no comando MODEL suprime testes univariados para cada uma das respostas individuais $\mathrm{d} 5, \mathrm{~d} 173, \mathrm{~d} 229, \mathrm{~d} 285, \mathrm{~d} 341$ e d435. O comando REPEATED produz um nome para os fatores intra-indivíduos no "output" resultante e especifica que as matrizes de soma de quadrados dos erros e produtos cruzados (SSCP) sejam exibidas juntamente com o teste de esfericidade. A interação entre os fatores entre e intra-indivíduos são automaticamente incluídas no modelo. Aqui testes multivariados são produzidos: Lambda de Wilks, Traço de Pillai, Traço de Hotelling-Lawley e Roy, além de apresentar as correções de Geisser-Greenhouse e Huynh-Feldt para os números de graus de liberdade.

Assim tem-se a matriz da soma de quadrados e produtos cruzados do resíduo

$$
\mathrm{E}=\left[\begin{array}{rrrrrr}
0,2474 & 0,3726 & 0,3349 & -0,0322 & 0,0948 & -0,0177 \\
0,3726 & 2,5024 & 2,1888 & -0,0831 & 0,7834 & 0,3153 \\
0,3349 & 2,1888 & 3,0302 & 0,0090 & 0,1224 & -0,0023 \\
-0,0321 & -0,0831 & 0,0090 & 1,2227 & 0,7591 & 0,8995 \\
0,0948 & 0,7834 & 0,1224 & 0,7591 & 1,1844 & 1,2297 \\
-0,0177 & 0,3153 & -0,0023 & 0,8995 & 1,2297 & 1,6683
\end{array}\right]
$$

bem como a matriz das correlações parciais dos resíduos, e os niveis descritivos associados aos testes das correlações parciais. 


$\mathbf{R}=\left[\begin{array}{rrrrrr}1,0000 & 0,4734 & 0,3868 & -0,0584 & 0,1403 & -0,0275 \\ 0,000 & 0,102 & 0,192 & 0,850 & 0,647 & 0,929 \\ 0,4734 & 1,0000 & 0,7949 & -0,0475 & 0,3647 & 0,1543 \\ 0,102 & 0,000 & 0,001 & 0,878 & 0,221 & 0,615 \\ 0,3868 & 0,7949 & 1,0000 & 0,0047 & 0,0518 & -0,0010 \\ 0,191 & 0,001 & 0,000 & 0,988 & 0,867 & 0,997 \\ -0,0585 & -0,0475 & 0,0047 & 1,0000 & 0,5055 & 0,6298 \\ 0,850 & 0,878 & 0,988 & 0,000 & 0,078 & 0,021 \\ 0,1403 & 0,3647 & 0,0518 & 0,5055 & 1,0000 & 0,7011 \\ 0,647 & 0,221 & 0,867 & 0,078 & 0,000 & 0,008 \\ -0,0275 & 0,1543 & -0,0010 & 0,6298 & 0,7011 & 1,0000 \\ 0,929 & 0,615 & 0,997 & 0,021 & 0,008 & 0,000\end{array}\right]$

Nas Tabelas 5, 6, 7 e 8 são apresentados os testes multivariados, o teste de esfericidade, os testes univariados com as correções para os números de graus de liberdade dos fatores intra-indivíduos e os testes sobre tendências, respectivamente.

Tabela 5. Testes multivariados para os fatores intra-indivíduos.

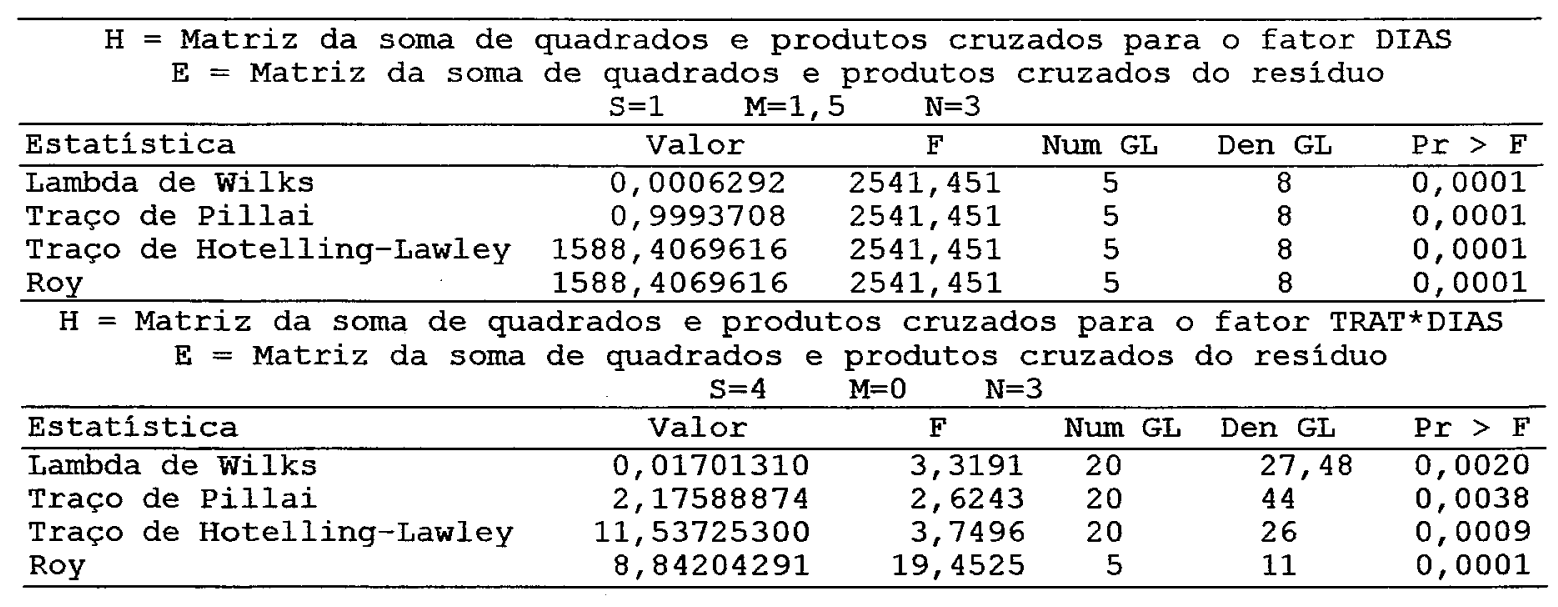

Observando os testes multivariados obtidos pelo proc GLM, apresentados na Tabela 5, pode-se concluir que o teste para o fator Dias, que testa a hipótese de perfis horizontais, é rejeitada pelos testes, indicando um crescimento significativo das distâncias 
médias ao longo do tempo para todos os tratamentos. $\mathrm{O}$ teste para a interação Tratamentos $\times$ Dias, que testa a hipótese de paralelismo, também é rejeitada pelos testes. Verifica-se ainda que os resultados foram os mesmos obtidos pelos testes univariados apresentados na Tabela 4.

Quanto ao teste de esfericidade, o resultado é apresentado na Tabela 6, onde pode-se observar que a condição de esfericidade foi violada com um nível de significância de 0,0320 , ou seja, a matriz de covariâncias não pode ser considerada do tipo Huynh-Feldt. Como essa suposição é violada, faz-se necessária a utilização de uma correção para os números de graus de liberdade dos fatores intra-indivíduos, nesse caso o fator Dias e a interação Tratamentos $\times$ Dias .

Tabela 6. Teste de Esfericidade.

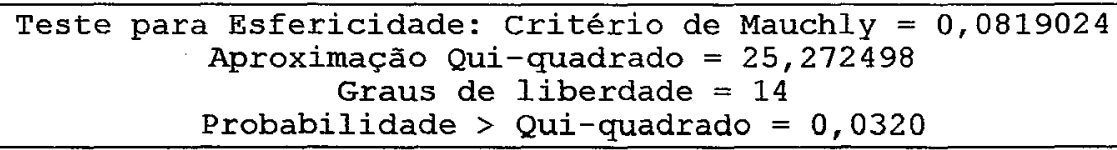

Tabela 7. Testes univariados e correções para o número de graus de liberdade para os efeitos intra-indivíduos.

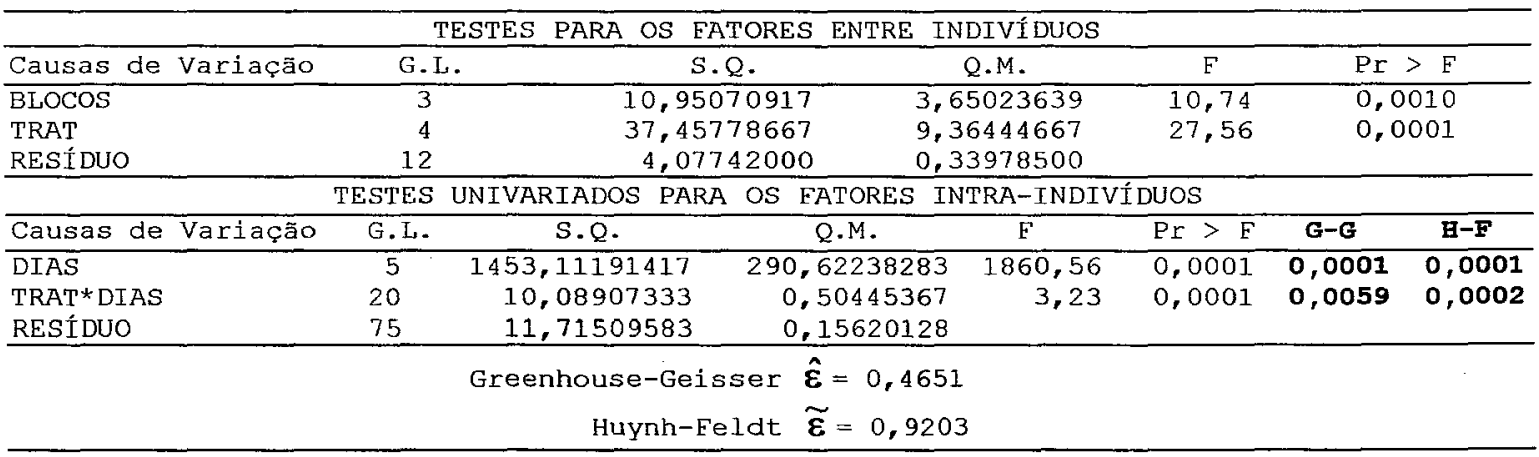

Através da Tabela 7, pode-se testar, da mesma forma que no caso multivariado, as hipóteses para perfis paralelos, horizontais e coincidentes. Assim, a hipótese de perfis coincidentes é verificada através do teste para o fator entre indivíduos, nesse caso, o fator Tratamento, sendo rejeitada, indicando que as distâncias médias dos tratamentos são diferentes. A hipótese de perfis paralelos, verificada através do teste para 
a interação Tratamentos $\times$ Dias, é rejeitada, o mesmo acontecendo com a hipótese de perfis horizontais obtida com o teste para o fator Dias. Essas conclusões foram as mesmas obtidas com os testes multivariados encontrados na Tabela 5. Porém nem sempre os resultados desses testes são equivalentes.

$\mathrm{Na}$ Tabela 7 , também são fornecidas as correções para os números de graus de liberdade dos testes $\mathrm{F}$ para os fatores intra-indivíduos. Como o teste de esfericidade foi rejeitado (Tabela 6), para tomada de decisão com relação às hipóteses, os níveis mínimos de significância em negrito é que foram utilizados para tomada de decisão. Um detalhe a ser observado é que, mesmo com as correções para os números de graus de liberdade, a significância dos testes não foi alterada.

Tabela 8. Testes sobre tendências.

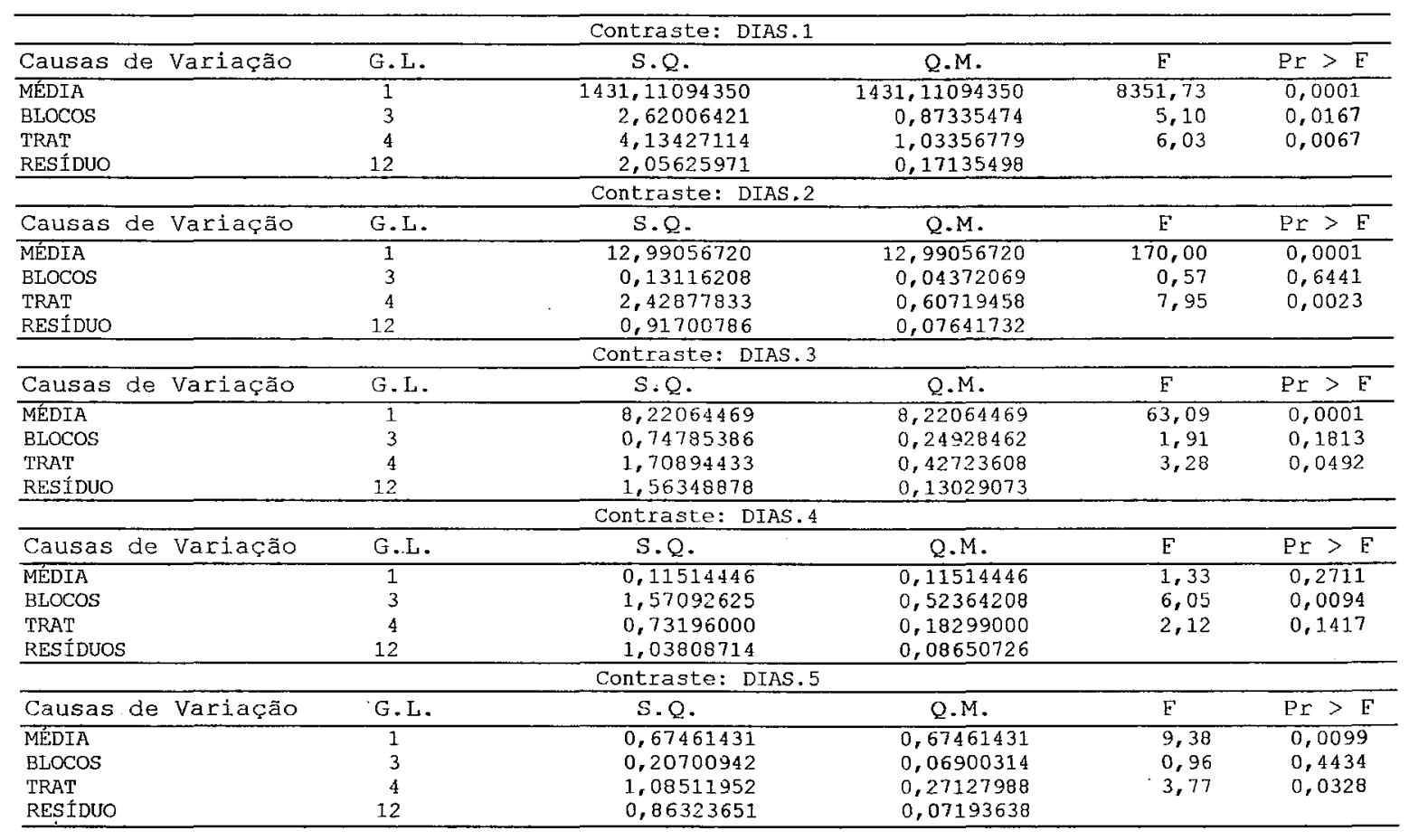

Os resultados dos testes para as tendências são apresentados na Tabela 8 , indicando que o comportamento dos perfis médios pode ser explicado por um polinômio de $5^{\underline{0}}$ grau, mas na prática não se teria explicação para um polinômio desse grau. Por esse 
motivo, optou-se pela tendência cúbica, que apresenta diferença entre os tratamentos com valor- $\mathrm{p}=0,0492$, e existe uma tendência cúbica do número médio de folhas sobre os dias (valor-p $=0,0001$ ). $O$ quarto grau é o componente de maior ordem não significativo, não existindo diferenças entre os tratamentos com um valor-p $=0,1417$.

São, então, essas as formas possíveis de se analisarem dados de medidas repetidas através do proc GLM.

Para analisar dados de medidas repetidas com o proc MIXED é preciso especificar todos os fatores entre e intra-individuos no comando MODEL, bem como a matriz de covariâncias no comando REPEATED com a opção TYPE=. A Opção $R$ mostra as estimativas dos componentes de variância associados à matriz escolhida. Os testes para efeitos fixos são similares aos testes univariados produzidos pelo proc GLM, sendo também produzidos testes multivariados quando a matriz de covariâncias sem estrutura (TYPE $=\mathrm{UN})$ é especificada. Esses testes são: Hotelling-Lawley-McKeon e Hotelling-Lawley-Pillai-Samson, e são obtidos com as opções HLM e HPLS no comando REPEATED.

O proc MIXED não apresenta o teste de esfericidade para a verificação da condição de H-F, mas produz informações para a construção de um teste de razão de verossimilhança. Para isso, são utilizados os seguintes comandos:

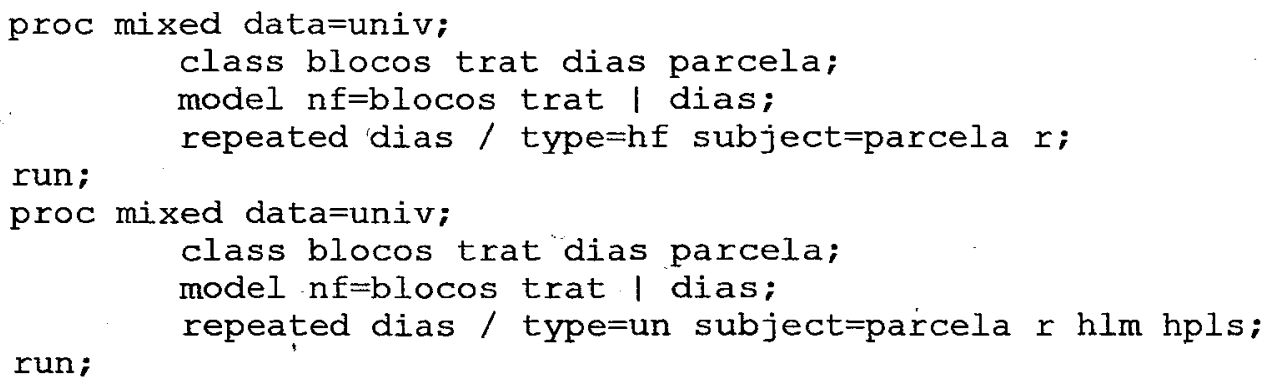

Com esse programa é possível produzir um teste de razão de verossimilhança para verificar a suposição de esfericidade. Para tanto, os seguintes passos devem ser observados: a) subtrair o valor da -2 REML $\log$ da função de verossimilhança para o modelo com a matriz de covariâncias sem estrutura de -2 REML log da função 
de verossimilhança para a estrutura Huynh-Feldt, b) calcular os graus de liberdade com a diferença dos números de parâmetros estimados para as duas estruturas, e c) comparar o resultado com a distribuição $\chi^{2}$ com os números de graus de liberdade obtidos no passo anterior.

O teste pode ser obtido com o auxílio dos resultados encontrados na Tabela 9. Então tem-se que $\chi^{2}=129,6241-88,4295=41,1946$, com $20-6=14$ graus de liberdade e com um nivel de significância de 0,0002. Rejeita-se, portanto, a hipótese de esfericidade.

Tabela 9. Informações sobre os modelos univariados com estruturas da matriz de covariâncias do tipo Huynh-Feldt e Sem Estrutura utilizando o proc MIXED.

\begin{tabular}{lr|lr}
\hline MATRIZ DE COVARIÂNCIAS - HUYNH-FELDT & MATRIZ DE COVARIÂNCIAS & - SEM ESTRUTURA \\
\hline Descrição & Valor & Descrição & Valor \\
Observaçes & 120,0000 & Observações & 120,0000 \\
Res. Log da Verossimilhança & $-64,8120$ & Res. Log da Verossimilhança & $-44,2148$ \\
Critério de Informação Akaike & $-71,8120$ & Critério de Informação Akaike & $-65,2148$ \\
Critéio de Schwarz & $-80,4427$ & Critéio de Schwarz & $-91,1068$ \\
-2 Res Log da Verossimilhança & 129,6241 & -2 Res Log da Verossimilhança & 88,4295 \\
Modelo Nulo LRT Qui-Quadrado & 19,2230 & Modelo Nulo LRT Qui-Quadrado & 60,4176 \\
Modelo Nulo LRT GI, & 6,0000 & Modelo Nulo LRT GL & 20,0000 \\
Modelo Nulo LRT p-valor & 0,0038 & Modelo Nulo LRT P-valor & 0,0000 \\
\hline
\end{tabular}

Na Tabela 10, encontram-se os testes para os efeitos fixos dos modelos, quando estimados com as matrizes de covariâncias do tipo Huynh-Feldt e Sem Estrutura. Pode-se observar que os números de graus de liberdade dos testes são diferentes para as duas estruturas, pelo fato de serem estimados via máxima verossimilhança restrita, que leva em conta o número de parâmetros da matriz de covariâncias estimada. Nesse caso, os testes obtidos através das duas estruturas de covariâncias apresentam os mesmos resultados, indicando que existe diferença entre os Tratamentos, os Dias e a interação Tratamentos $\times$ Dias. 
Tabela 10. Testes para os efeitos fixos dos modelos com estruturas para a matriz de covariâncias do tipo Huynh-Feldt e Sem Estrutura utilizando o proc MIXED.

\begin{tabular}{|c|c|c|c|c|c|}
\hline \multicolumn{6}{|c|}{ MATRIZ DE COVARIÂNCIAS - HUYNH-FELDT } \\
\hline Causas de Variação & GL Numerador & GL & Denominador & $\mathrm{F}$ & Pr $>F$ \\
\hline BLOCOS & 3 & & 12 & 27,06 & 0,0001 \\
\hline TRAT & 4 & & 12 & 14,02 & 0,0002 \\
\hline DIAS & 5 & & 75 & 1860,56 & 0,0001 \\
\hline TRAT *DIAS & 20 & & 75 & 3,23 & 0,0001 \\
\hline \multicolumn{6}{|c|}{ MATRIZ DE COVARIÂNCIAS - SEM ESTRUTURA } \\
\hline Causas de Variação & GL Numerador & GL & Denominador & F & $\mathrm{Pr}>\mathrm{E}$ \\
\hline BLOCOS & 3 & & 12 & 27,06 & $0,0 \overline{001}$ \\
\hline TRAT & 4 & & 12 & 13,35 & 0,0002 \\
\hline DIAS & 5 & & 12 & 1504,09 & 0,0001 \\
\hline TRAT*DIAS & 20 & & 12 & 4,17 & 0,0072 \\
\hline
\end{tabular}

A seguir, na Tabela 11, encontram-se os testes multivariados produzidos pelo proc MIXED.

Para dados balanceados, o modelo básico é equivalente ao modelo multivariado de medidas repetidas do proc GLM. Esses testes são produzidos somente quando a matriz de covariâncias sem estrutura (TYPE=UN) é especificada, sendo os resultados diferentes daqueles obtidos com a utilização do proc GLM e apresentados na Tabela 5. Nesse caso a estatística de Hotelling-Lawley-McKeon não rejeita a hipótese para a interação Tratamentos $\times$ Dias.

Tabela 11. Testes multivariados produzidos pelo proc MIXED.

\begin{tabular}{ccccrrr}
\hline \multicolumn{7}{c}{ Estatística de Hotelling-Lawley-McKeon } \\
\hline Causas de Variação & GL Numerador & GL & Denominador & F & Pr > F \\
\hline DIAS & 5 & 8 & 1002,72 & 0,0001 \\
TRAT DIAS & 20 & 11,5 & 2,53 & 0,0546 \\
\hline \multicolumn{7}{c}{ Estatística de Hotelling-Lawley-Pillai-Samson } \\
\hline Causas de Variação & GL Numerador & GL Denominador & F & Pr $>$ F \\
\hline DIAS & 5 & 8 & 1002,72 & 0,0001 \\
TRAT*DIAS & 20 & 26 & 2,26 & 0,0259 \\
\hline
\end{tabular}

Com o proc MIXED é possivel trabalhar com vários tipos de modelos. Esse mesmo conjunto de dados pode ser analisado, por exemplo, como um modelo de 
curva de crescimento onde o fator Dias é considerado uma covariável. Já a análise de curvas de crescimentos, tem-se interesse na relação entre à resposta (Número Médio de Folhas) e ao fator repetido (Dias).

Antes de examinar a significância dos efeitos fixos, deve-se estar certo de que a estrutura da matriz de covariâncias é adequada, pois os testes para os efeitos fixos tornam-se inválidos se a estrutura da matriz de covariâncias for mal especificada.

Os comandos utilizados são os mesmos discutidos anteriormente, mudando apenas o modelo, que agora considera o fator Dias como uma covariável. Mas, para que se tenha uma idéia do grau do polinômio a ser ajustado, deve-se observar o gráfico de perfis médios, apresentado na Figura 6. Esse gráfico sugere que um polinômio de grau 3 pode ser suficiente para explicar o comportamento dos dados .

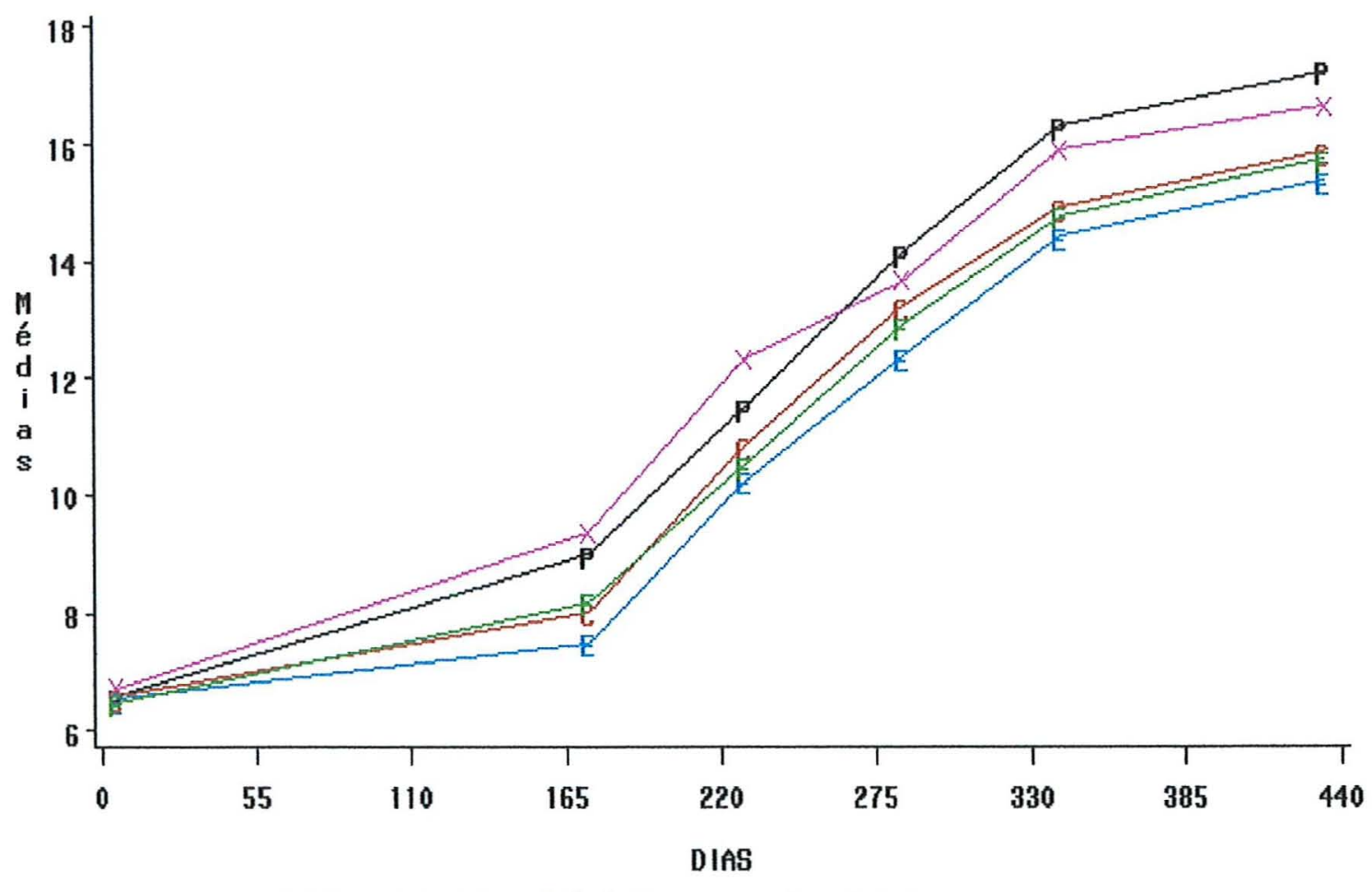

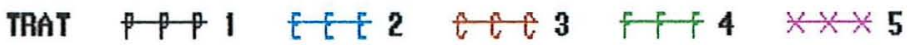

Figura 6. Perfis médios dos tratamentos ao longo dos dias. 
O modelo que será utilizado é apresentado a seguir:

$\mathrm{NMF}=$ Blocos + Trat + Dias + Trat $\times$ Dias $+\operatorname{Dias}^{2}+\operatorname{Trat} \times \operatorname{Dias}^{2}+\operatorname{Dias}^{3}+\operatorname{Trat} \times \operatorname{Dias}^{3}$

Resultados para esse modelo podem ser obtidos da seguinte forma:

proc mixed data=univ;

class blocos trat parcela;

model $\mathrm{nmf}=\mathrm{blocos}$ trat dias trat*dias dias*dias trat*dias*dias

dias*dias*dias trat*dias*dias*dias;

run; repeated / type=un subject=parcela ;

Para esse modelo várias estruturas da matriz de covariâncias devem ser verificadas, e aquela que apresentar o maior AIC será a escolhida. Isso pode ser feito através de uma macro do SAS que agiliza essa tarefa. A seguir são apresentadas as linhas de comando dessa macro e uma forma de ordenar os resultados obtidos.

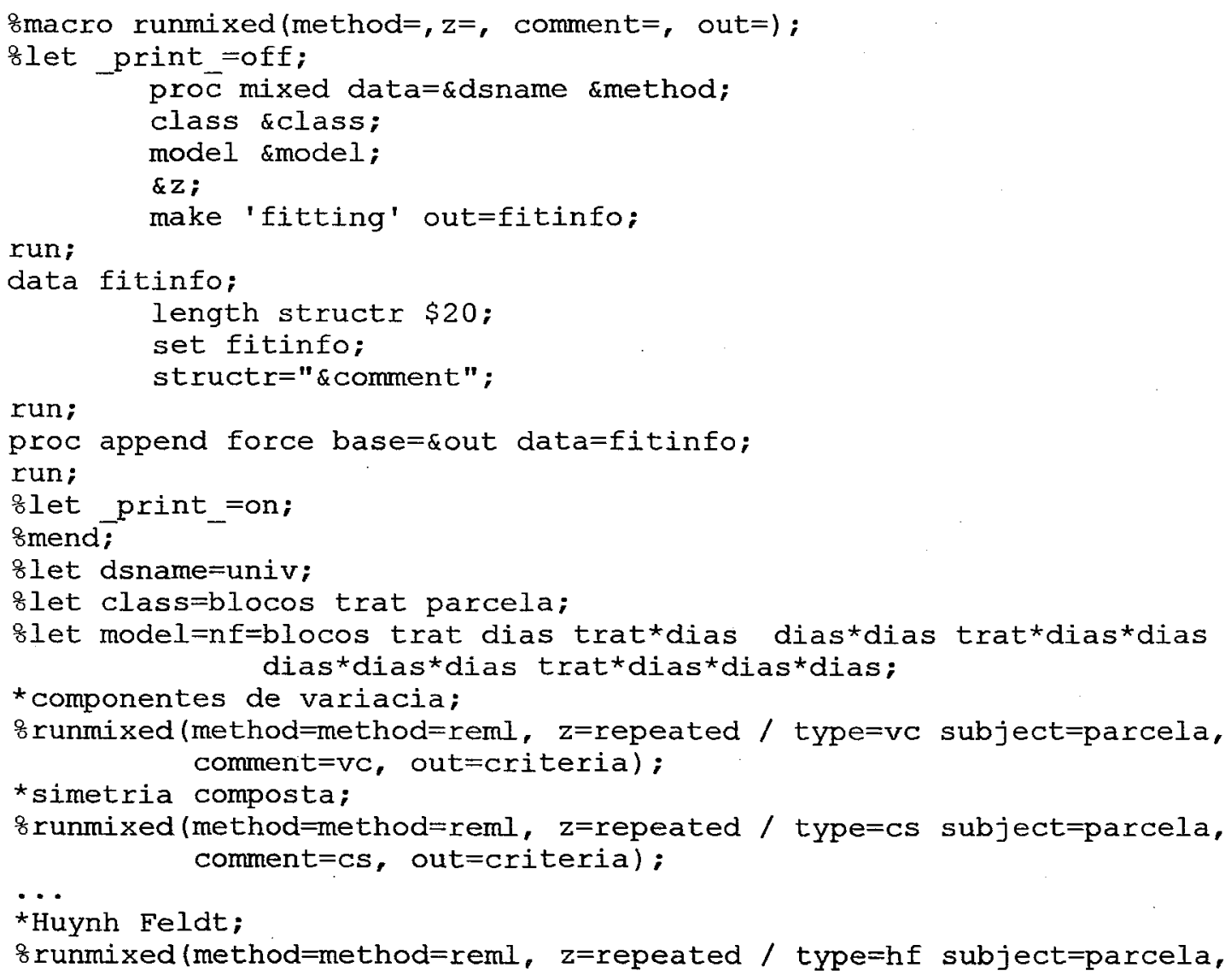




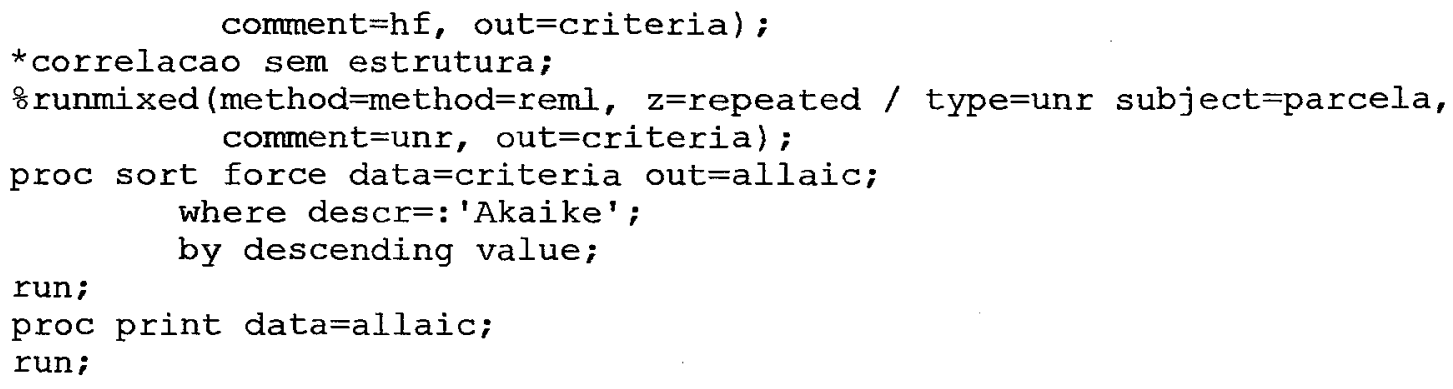

Executando esses comandos obtêm-se os resultados dos AIC (Critério de Informação de Akaike) em ordem decrescente. Deve-se, então, escolher aquele que apresentar o maior valor. As estruturas utilizadas aqui foram apresentadas em seções anteriores do trabalho, e os valores do AIC são apresentados na Tabela 12.

Tabela 12. Critério de Informação de Akaike (AIC) para o modelo completo.

\begin{tabular}{lc}
\hline Estruturas da Matriz de Covariâncias & AIC \\
\hline Sem Estrutura & $-231,011^{\prime}$ \\
Sem Estrutura com Correlação & $-231,011$ \\
Primeira Antedependência & $-231,928$ \\
Estrutura Fator Analítico & $-233,492$ \\
Auto-regressiva de 1 ordem Heterogênea & $-234,602$ \\
Toeplitz & $-235,202$ \\
Toeplitz Heterogênea & $-235,680$ \\
Huynh-Feldt & $-237,077$ \\
Auto-regressiva de 1 ${ }^{a}$ ordem & $-237,487$ \\
Toeplitz "Banded" - 2 & $-237,680$ \\
Auto-regressiva de 1 ${ }^{a}$ Ordem Médias Móveis & $-238,399$ \\
Simetria Composta & $-239,816$ \\
Componente de Variancia & $-240,328$ \\
Simetria Composta Heterogênea & $-241,147$ \\
Diagonal Principal "Banded" & $-241,672$ \\
\hline
\end{tabular}

A estrutura escolhida foi a do tipo UN (sem estrutura), sendo agora necessária a verificação dos testes para os efeitos fixos, e caso algum efeito não seja significativo deverá ser retirado do modelo. Na Tabela 13 são apresentados os testes para os efeitos fixos do modelo completo, onde o efeito Tratamentos $\times$ Dias $^{3}$ não é significativo, podendo ser retirado do modelo. 
Tabela 13. Testes para os efeitos fixos do modelo completo com a estrutura da matriz de covariâncias do tipo Sem Estrutura.

\begin{tabular}{cccrc}
\hline Causas de Variação & GL Numerador & GL Denominador & \multicolumn{1}{c}{ F } & Pr $>$ F \\
\hline BLOCOS & 3 & 12 & 27,06 & 0,0001 \\
TRAT & 4 & 12 & 0,68 & 0,6183 \\
DIAS & 1 & 12 & 744,41 & 0,0001 \\
TRAT $^{\star}$ DIAS & 4 & 12 & 5,17 & 0,0117 \\
DIAS $^{2}$ & 1 & 12 & 2025,65 & 0,0001 \\
TRAT $^{*}$ DIAS $^{2}$ & 4 & 12 & 3,04 & 0,0605 \\
DIAS $^{3}$ & 1 & 12 & 447,94 & 0,0001 \\
TRAT $^{\star}$ DIAS $^{3}$ & 4 & 12 & 2,63 & 0,0867 \\
\hline
\end{tabular}

Como o efeito Tratamentos $\times$ Dias $^{3}$ foi retirado do modelo, deve-se verificar novamente o que ocorre com as estruturas da matriz de covariâncias.

Os novos valores do AIC para o modelo reduzido são apresentados na Tabela 14.

Tabela 14. Critério de Informação de Akaike para o modelo reduzido.

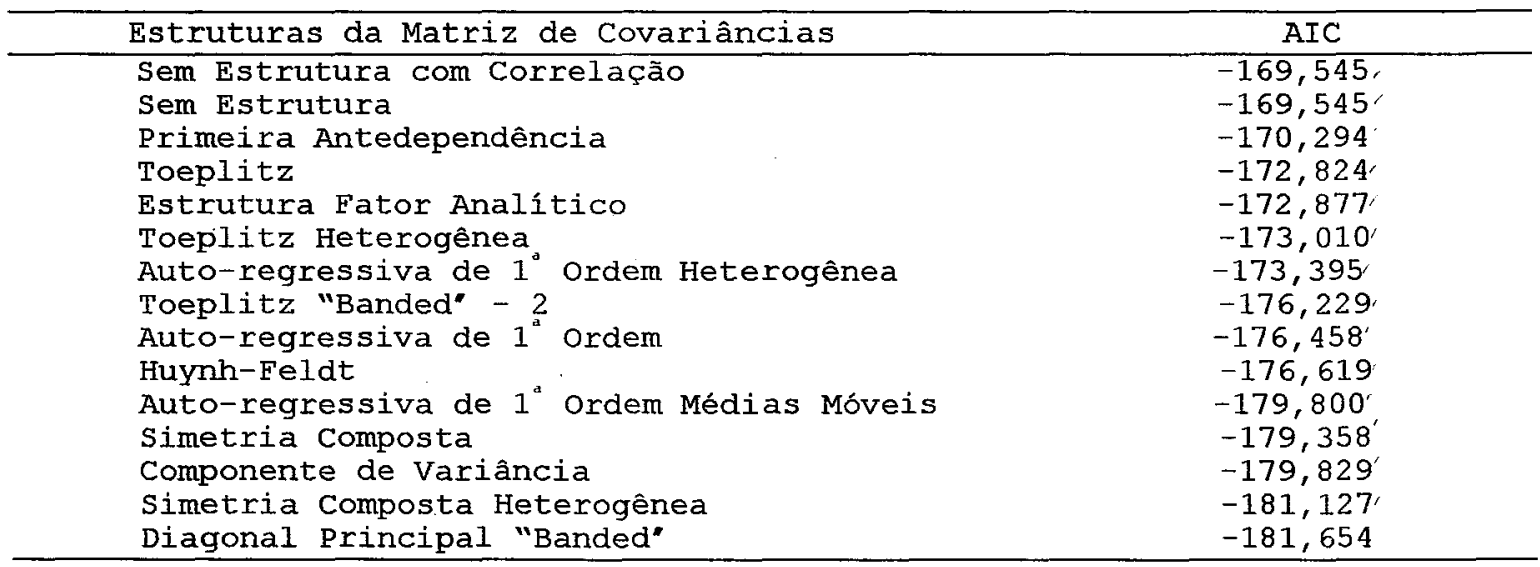

Sendo escolhida dessa forma a estrutura da matriz de covariâncias do tipo UNR (Sem Estrutura com Correlações) por apresentar o maior valor de AIC.

$\mathrm{Na}$ Tabela 15 são apresentados os testes para os efeitos fixos do modelo reduzido. Como todos os efeitos são significativos, nenhum será retirado do modelo. 
Tabela 15. Testes para os efeitos fixos do modelo reduzido com a estrutura da matriz de covariâncias do tipo Sem Estrutura com Correlações.

\begin{tabular}{|c|c|c|c|c|}
\hline Causas de Variação & GL Numerador & GL Denominador & $\mathrm{F}$ & $\operatorname{Pr}>F$ \\
\hline BLOCOS & 3 & 12 & 27,06 & 0,0001 \\
\hline TRAT & 4 & 12 & 0,63 & 0,6480 \\
\hline DIAS & 1 & 12 & 585,55 & 0,0001 \\
\hline TRAT $\star D I A S$ & 4 & 12 & 12,31 & 0,0003 \\
\hline $\mathrm{DIAS}^{2}$ & $i$ & 12 & 1588,64 & 0,0001 \\
\hline TRAT $* D I A S^{2}$ & 4 & 12 & 6,63 & 0,0047 \\
\hline $\operatorname{DIAS}^{3}$ & 1 & 12 & 1601,54 & 0,0001 \\
\hline
\end{tabular}

Pode-se, então, realizar testes para verificar se os efeitos dos tratamentos podem ser considerados sem diferenças, ou seja, se uma única curva pode explicar o comportamento de mais de um tratamento. Esses testes, no proc MIXED, são realizados pelo comando LSMEANS. No caso o teste utilizado foi o de Tukey-Kramer. Também é possível obter as estimativas para os parâmetros do modelo através da opção $\mathrm{S}$ no comando MODEL, apresentados no programa a seguir:

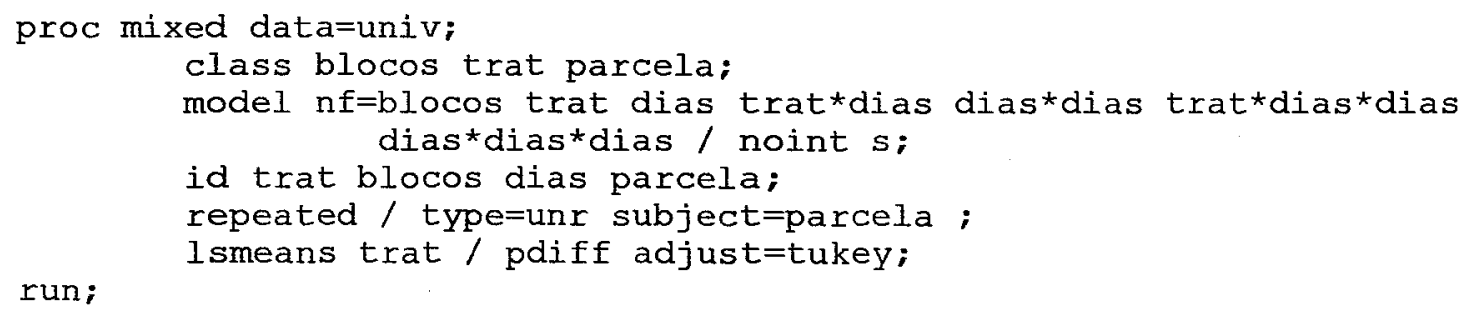

O teste de Tukey-Kramer para os tratamentos (Tabela 16) mostra que as comparações entre os tratamentos T1 e T5, T3 e T4 não são significativas, sugerindo que é possível unir os tratamentos T1 (casca de Pinus + turfa + perlita) e T5 (Xaxim + turfa + perlita) e os tratamentos T3 (Coxim + turfa + perlita) e T4 (Fibra de coco + turfa + perlita). Sendo assim, têm-se 3 curvas explicando o comportamento dos 5 tratamentos. 
Tabela 16. Teste de Tukey-Kramer para o fator tratamentos do modelo reduzido com a estrutura da matriz de covariâncias do tipo Sem Estrutura com Correlações.

\begin{tabular}{crcrrc}
\hline TRATAMENTOS & DIFERENÇAS & ERRO PADRÃO & GL & \multicolumn{1}{c}{ t } & Pr $>|t|$ \\
\hline T1 - T2 & 1,34276669 & 0,19973087 & 13 & 6,72 & 0,0001 \\
T1 - T3 & 0,71136001 & 0,19973087 & 13 & 3,56 & 0,0035 \\
T1 - T4 & 0,96613642 & 0,19973087 & 13 & 4,84 & 0,0003 \\
T1 - T5 & 0,11570061 & 0,19973087 & 13 & 0,58 & 0,5723 \\
T2 - T3 & $-0,63140668$ & 0,19973087 & 13 & $-3,16$ & 0,0075 \\
T2 - T4 & $-0,37663027$ & 0,19973087 & 13 & $-1,89$ & 0,0819 \\
T2 - T5 & $-1,22706608$ & 0,19973087 & 13 & $-6,14$ & 0,0001 \\
T3 - T4 & 0,25477641 & 0,19973087 & 13 & 1,28 & 0,2244 \\
T3 - T5 & $-0,59565940$ & 0,19973087 & 13 & $-2,98$ & 0,0106 \\
T4 - T5 & $-0,85043581$ & 0,19973087 & 13 & $-4,26$ & 0,0009 \\
\hline
\end{tabular}

Depois de realizar os testes para o modelo obtiveram-se as seguintes curvas de crescimento, que são apresentadas na Figura 7.

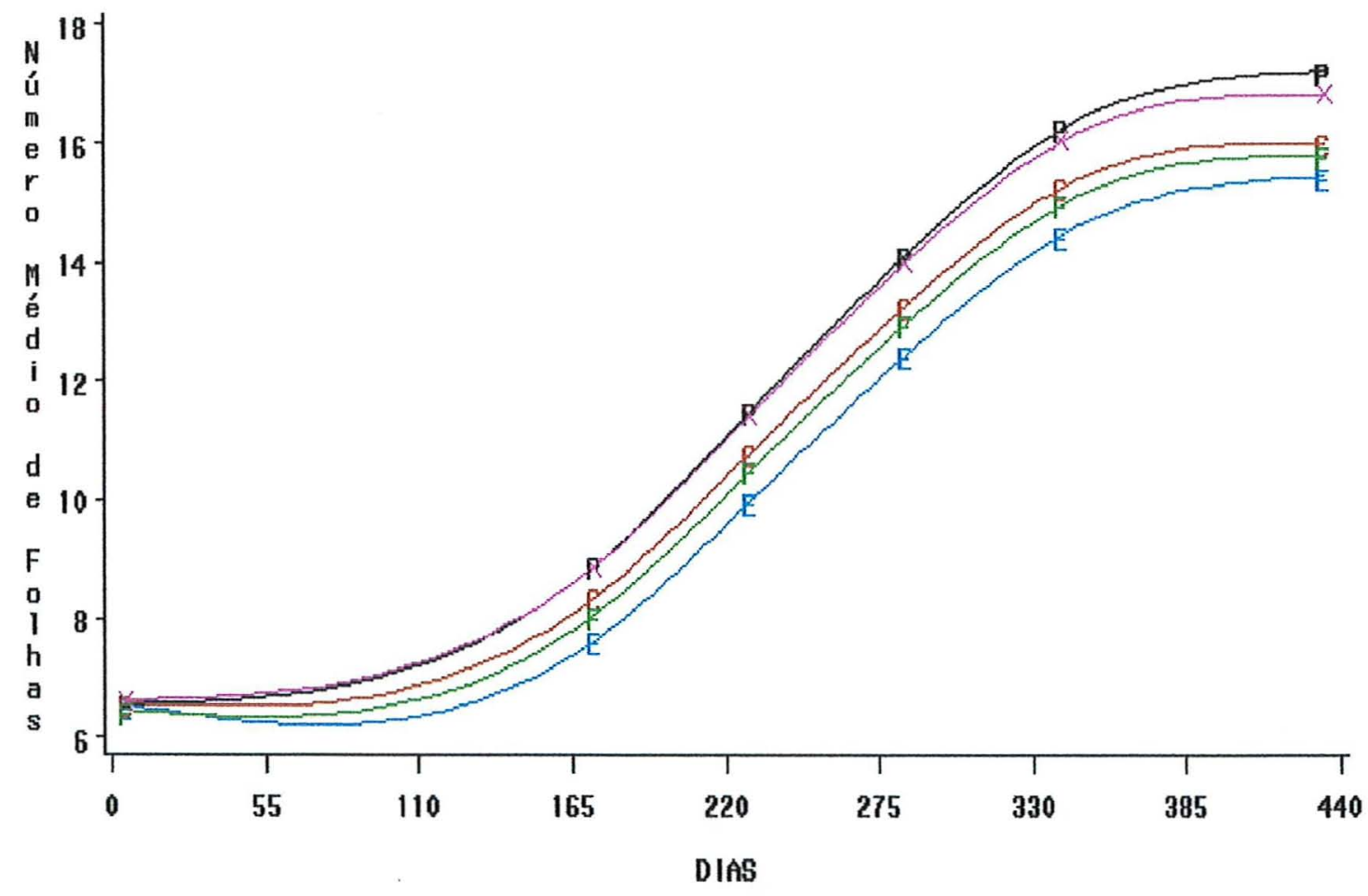

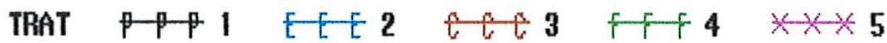

Figura 7. Modelo ajustado para os tratamentos ao longo dos dias. 
Observando-se a Figura 7 e sendo justificado pelo teste de Tukey-Kramer (Tabela 16), não há diferença estatisticamente significativa para os tratamentos Casca de Pimus + turfa + perlita e Xaxim + turfa + perlita (T1 e T5), para os tratamentos Coxim + turfa + perlita e Fibra de coco + turfa + perlita (T3 e T4). Sendo assim, 3 curvas podem explicar o comportamento dos 5 tratamentos. Os modelos estimados foram:

$$
\begin{gathered}
\text { T1 e T5 }=6,739584-0,033190 * \text { Dias }+0,000351 * \operatorname{Dias}^{2}-0,000001 * \operatorname{Dias}^{3} \\
\text { T2 }=6,692766-0,043021 * \text { Dias }+0,000365 * \operatorname{Dias}^{2}-0,000001 * \operatorname{Dias}^{3} \\
\text { T3e T4 }=6,693276-0,037557 * \text { Dias }+0,000355 * \operatorname{Dias}^{2}-0,000001 * \operatorname{Dias}^{3}
\end{gathered}
$$

A verificação do ajuste do modelo pode ser realizada através da análise gráfica dos resíduos (Figuras 8 e 9), que permitem afirmar que o modelo apresentou um ajuste razoável. Os gráficos mostram que os resíduos têm distribuição normal e que não foram observados "outliers".

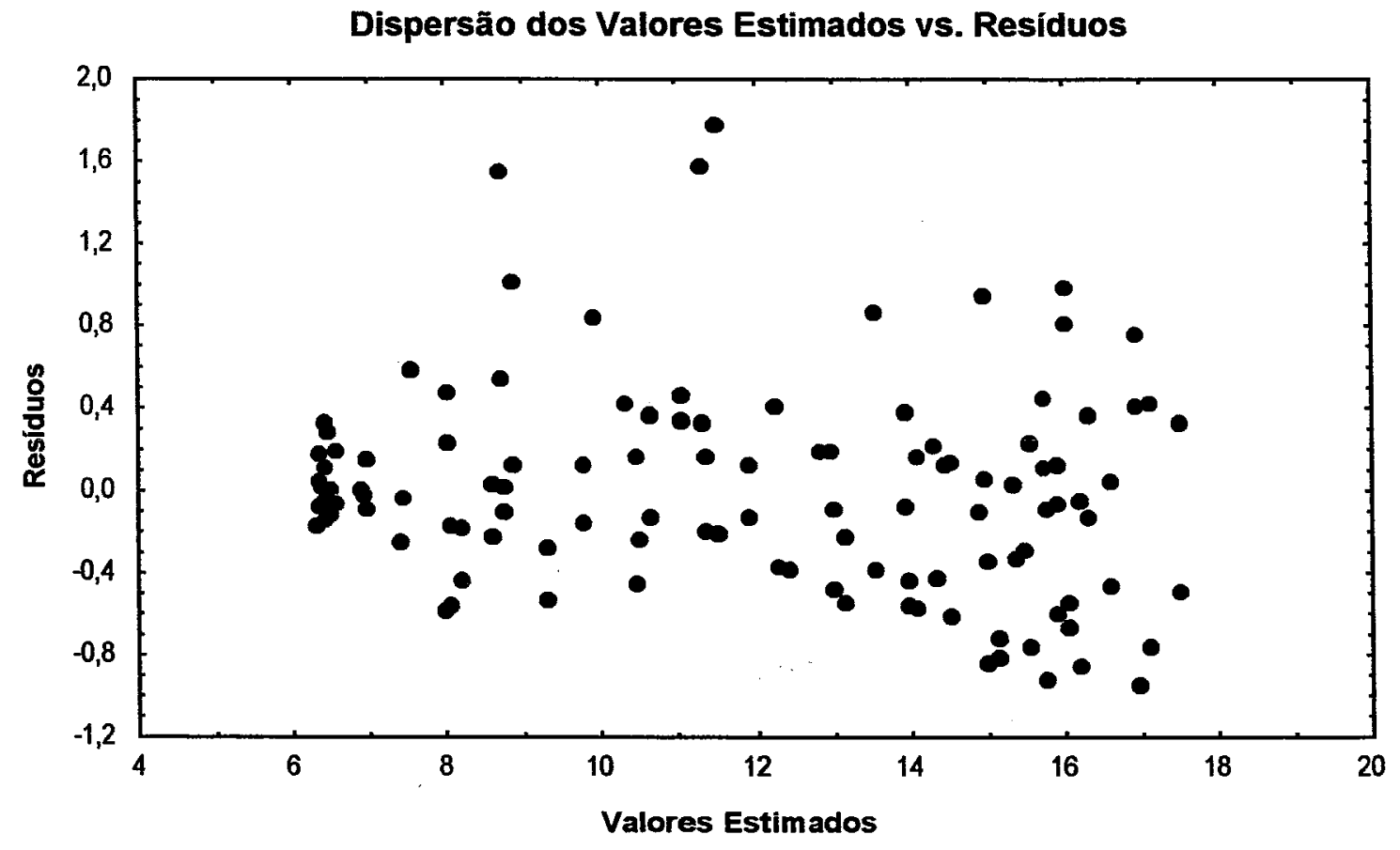

Figura 8. Dispersão dos valores estimados vs. resíduos. 


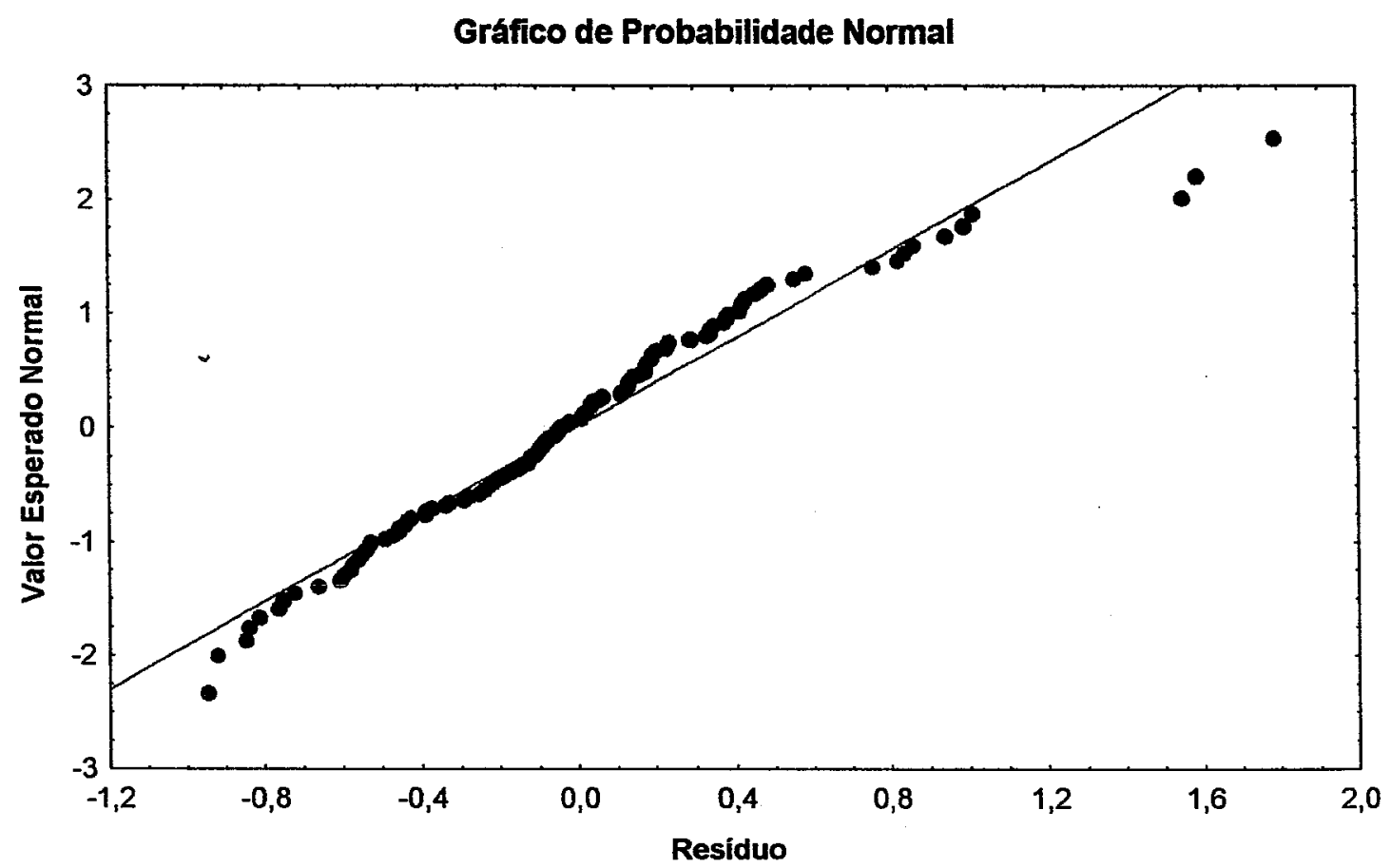

Figura 9. Gráfico de probabilidade normal.

As estimativas dos parâmetros das curvas de crescimento para o modelo escolhido são apresentadas na Tabela 17 , e as curvas de crescimento apresentadas na Figura 10, onde T1 é a curva de crescimento para Casca de Pinus + turfa + perlita e Xaxim + turfa + perlita, T2 é a curva para Casca de Eucaliptos e T3 para Coxim + turfa + perlita e Fibra de coco + turfa + perlita.

Tabela 17. Estimativas do modelo final ajustado.

\begin{tabular}{|c|c|c|c|c|c|c|c|}
\hline EFEITOS & BLOCOS & TRAT & ESTIMATIVAS & ERRO PADRÃO & GL & $t$ & $\operatorname{Pr}>|t|$ \\
\hline BLOCOS & 1 & & 6,66579226 & 0,06858996 & 14 & 97,18 & 0,0001 \\
\hline BLOCOS & 2 & & 6,49860808 & 0,06858996 & 14 & 94,75 & 0,0001 \\
\hline BLOCOS & 3 & & 6,53154954 & 0,06858996 & 14 & 95,23 & 0,0001 \\
\hline BLOCOS & 4 & & 7,07715326 & 0,06858996 & 14 & 103,18 & 0,0001 \\
\hline TRAT & & 1 & 0,04630970 & 0,06887007 & 14 & 0,67 & 0,5123 \\
\hline TRAT & & 2 & $-0,00050911$ & 0,08434827 & 14 & $-0,01$ & 0,9953 \\
\hline TRAT & & 3 & 0,00000000 & & & & \\
\hline TRAT*DIAS & & 1 & $-0,03319041$ & 0,00159272 & 14 & $-20,84$ & 0,0001 \\
\hline TRAT*DIAS & & 2 & $-0,04302124$ & 0,00177231 & 14 & $-24,27$ & 0,0001 \\
\hline TRAT *DIAS & & 3 & $-0,03755778$ & 0,00159272 & 14 & $-23,58$ & 0,0001 \\
\hline TRAT * DIAS $^{2}$ & & 1 & 0,00035145 & 0,00000888 & 14 & 39,59 & 0,0001 \\
\hline TRAT*DIAS ${ }^{2}$ & & 2 & 0,00036575 & 0,00000901 & 14 & 40,59 & 0,0001 \\
\hline TRAT $^{*}$ DIAS $^{2}$ & & 3 & 0,00035543 & 0,00000888 & 14 & 40,04 & 0,0001 \\
\hline DIAS & & & $-0,00000051$ & , & I & 1 & 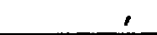 \\
\hline
\end{tabular}




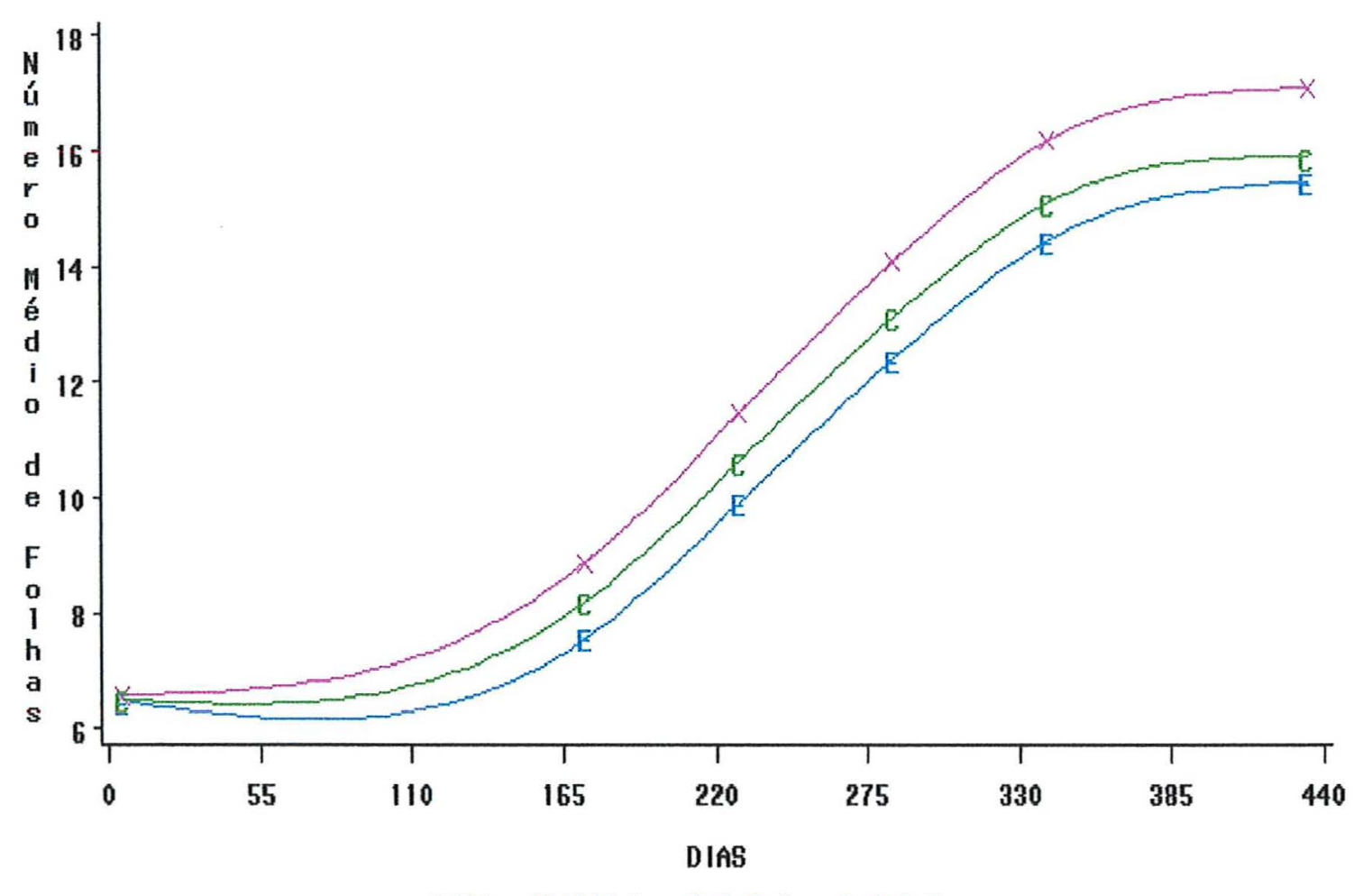

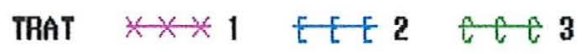

Figura 10. Modelo final ajustado para os tratamentos ao longo dos dias.

Considerando que o Xaxim está ficando escasso, o objetivo deste experimento era encontrar um substrato que apresentasse resultados semelhantes ou melhores para as plantas, neste caso bromélias. E com os resultados obtidos pode-se considerar que o substrato contendo Casca de Pinus apresentou resultados semelhantes aos do Xaxim, quando utilizada a proporção (5:4:1) para o substrato.

\subsection{Simulações}

Os experimentos simulados no SAS levaram em conta o delineamento de blocos ao acaso no esquema de parcelas subdivididas no tempo, com 4 blocos, 5 tratamentos e 6 tempos. 
No total foram considerados 80 casos para as simulações, levando em conta as combinações entre a distribuição normal com as 8 estruturas da matriz de covariâncias, efeitos nulos e não nulos, dados balanceados e desbalanceados. Da mesma forma, para a distribuição normal contaminada com suas respectivas ponderações. Para cada caso, 1.000 experimentos foram simulados. Detalhes sobre esses itens são apresentados a seguir.

Com relação aos efeitos dos fatores:

$\beta_{\mathrm{j}}$ : efeitos de blocos com $\mathrm{j}=1, \ldots, 4$;

$\alpha_{i}$ : efeitos de tratamentos com $i=1, \ldots, 5$;

$\tau_{\mathrm{k}}:$ efeitos de tempos com $\mathrm{k}=1, \ldots, 6 \mathrm{e}$

$\alpha \tau_{\text {ik }}:$ efeitos da interação tratamentos*tempos,

dois casos foram observados:

\section{Efeitos nulos:}

- $\beta_{1}=\beta_{2}=\beta_{3}=\beta_{4}=0$;

- $\alpha_{1}=\alpha_{2}=\alpha_{3}=\alpha_{4}=\alpha_{5}=0$;

- $\tau_{1}=\tau_{2}=\tau_{3}=\tau_{4}=\tau_{5}=\tau_{6}=0$;

- $\alpha \tau_{11}=\alpha \tau_{12}=\alpha \tau_{13}=\alpha \tau_{14}=\alpha \tau_{15}=\alpha \tau_{16}=\alpha \tau_{21}=\alpha \tau_{22}=\alpha \tau_{23}=\alpha \tau_{24}=\alpha \tau_{25}=\alpha \tau_{26}=\alpha \tau_{31}$ $=\alpha \tau_{32}=\alpha \tau_{33}=\alpha \tau_{34}=\alpha \tau_{35}=\alpha \tau_{36}=\alpha \tau_{41}=\alpha \tau_{42}=\alpha \tau_{43}=\alpha \tau_{44}=\alpha \tau_{45}=\alpha \tau_{46}=\alpha \tau_{51}=$ $\alpha \tau_{52}=\alpha \tau_{53}=\alpha \tau_{54}=\alpha \tau_{55}=\alpha \tau_{56}=0$.

\section{Efeitos não nulos:}

- $\beta_{1}=0,108, \beta_{2}=0,538, \beta_{3}=-0,438, \beta_{4}=0$;

- $\alpha_{1}=1,045, \alpha_{2}=-0,856, \alpha_{3}=-0,065, \alpha_{4}=-0,480, \alpha_{5}=0$;

- $\tau_{1}=-9,945, \tau_{2}=-7,288, \tau_{3}=-4,320, \tau_{4}=-2,988, \tau_{5}=-0,748, \tau_{6}=0$;

- $\alpha \tau_{11}=-0,74, \quad \alpha \tau_{12}=-0,963, \quad \alpha \tau_{13}=-1,428, \quad \alpha \tau_{14}=-0,125, \quad \alpha \tau_{15}=-0,188, \quad \alpha \tau_{16}=0$, $\alpha \tau_{21}=1,105, \alpha \tau_{22}=-0,583, \alpha \tau_{23}=-0,803, \alpha \tau_{24}=-0,01, \alpha \tau_{25}=-0,203, \alpha \tau_{26}=0, \alpha \tau_{31}=0,645$, 


$$
\begin{aligned}
& \alpha \tau_{32}=-0,545, \alpha \tau_{33}=-0,70, \alpha \tau_{34}=0,363, \alpha \tau_{35}=-0,2, \alpha \tau_{36}=0, \alpha \tau_{41}=0,668, \alpha \tau_{42}=-0,273, \\
& \alpha \tau_{43}=-0,898, \alpha \tau_{44}=0,148, \alpha \tau_{45}=-0,218, \alpha \tau_{46}=0, \alpha \tau_{51}=0, \alpha \tau_{52}=0, \alpha \tau_{53}=0, \alpha \tau_{54}=0, \\
& \alpha \tau_{55}=0 \text { e } \alpha \tau_{56}=0 .
\end{aligned}
$$

O desbalanceamento dos dados também foi estudado, pois foram simulados experimentos balanceados e desbalanceados com uma casela vazia, onde as observações para $\mathrm{i}=2, \mathrm{k}=2 \mathrm{e} \mathrm{j}=1,2,3$ e 4 foram retiradas.

São apresentadas na Tabela 18 , as estruturas das matrizes de covariâncias utilizadas para as simulações com a distribuição normal e a distribuição normal contaminada. Na primeira coluna da Tabela 18 encontram-se as matrizes utilizadas para a simulação de dados da distribuição normal. Com relação à simulação de dados da distribuição normal contaminada as duas colunas foram utilizadas, sendo que para as matrizes da primeira coluna foram usadas as seguintes ponderações $0,05,0,10,0,15 \mathrm{e}$ 0,20 , e para as matrizes da segunda coluna, as ponderações $0,95,0,90,0,85$ e 0,80 , respectivamente.

As estruturas utilizadas foram: VC - Componentes de variâncias; CS - Simetria composta; HF - Huynh-Feldt; UN(1) - Diagonal principal "banded"; UN Desestruturada; UNR - Desestruturada com correlações; TOEP(2) - Toeplitz "banded" e AR(1) - Auto regressiva de primeira ordem. 
Tabela 18. Estruturas da matriz de covariâncias utilizadas para as simulações.

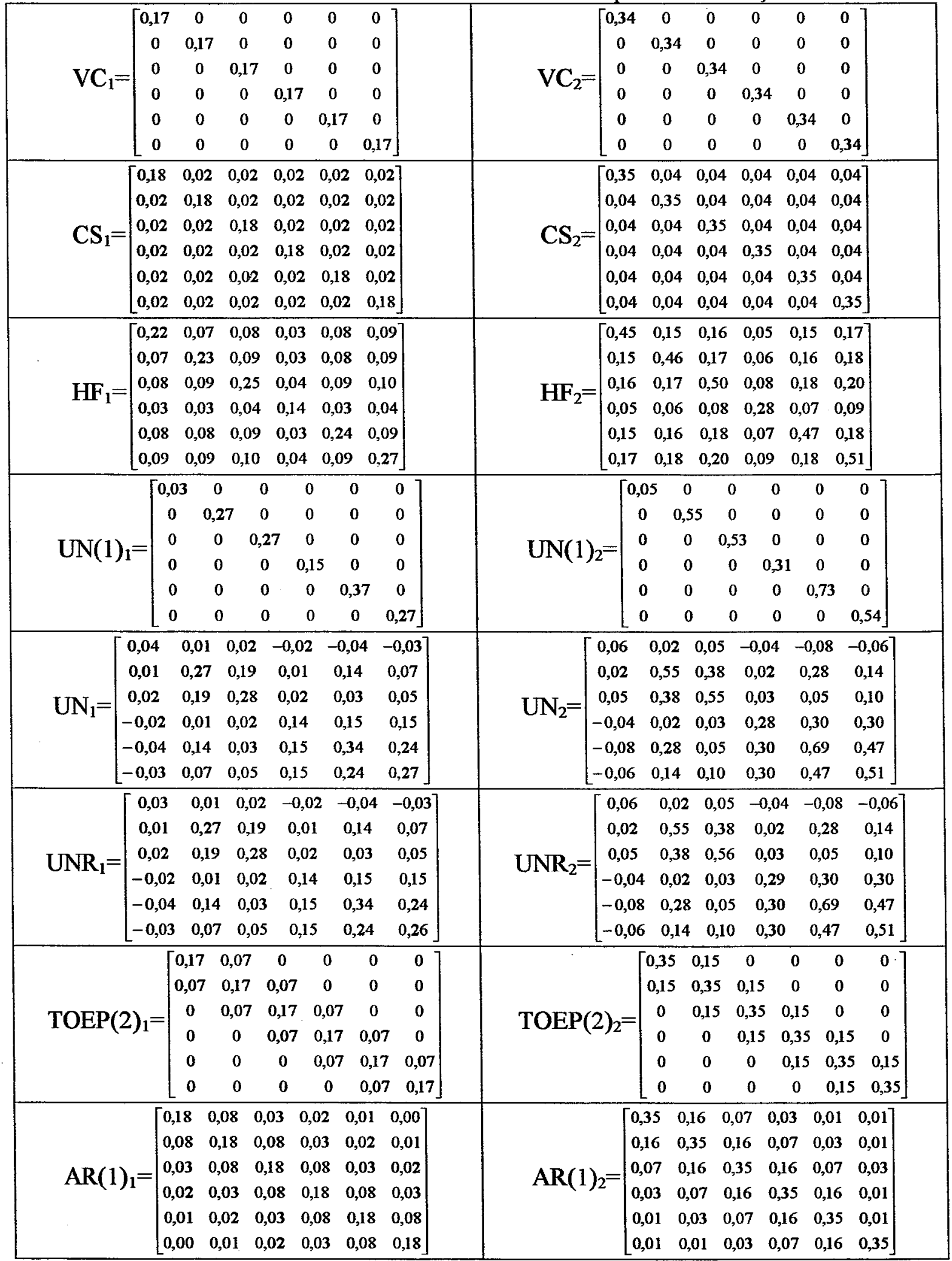


Para os casos com efeitos nulos, são apresentados nas Tabelas 19 e 20 os resultados da estatística $\chi^{2}$ para o teste de aderência da distribuição de freqüência dos níveis mínimos de significância, dos valores da estatística $F$ para os fatores Dias (subparcela) e da interação Tratamentos $\times$ Dias (parcelaxsubparcela), à distribuição uniforme $(0,1)$.

Os resultados apresentados na Tabela 19 são referentes aos simulados com a distribuição normal, e os da Tabela 20 com a distribuição normal contaminada.

Os valores da estatística $\chi^{2}$ das Tabelas 19 e 20 são comparados com o valor tabelado da estatística $\chi_{(19 ; 0,05)}^{2}=30,14$. A hipótese de aderência é rejeitada com $5 \%$ de probabilidade se $\chi_{\text {(Calculado) }}^{2}>\chi_{\text {(Tabelado) }}^{2}=30,14$.

Tabela 19. Valores da estatística $\chi^{2}$ para o teste de aderência da distribuição de freqüência dos níveis mínimos de significância associados aos valores da estatística $\mathrm{F}$ para Dias e interação Tratamentos $\times$ Dias, com dados balanceados e desbalanceados, para a distribuição normal.

\begin{tabular}{|c|c|c|c|c|c|c|c|c|c|}
\hline & \multirow{2}{*}{$\begin{array}{c}\text { Estatistica } \\
\text { F } \\
\end{array}$} & \multicolumn{8}{|c|}{ Estruturas da matriz de covariâncias } \\
\hline & & VC & $\mathrm{CS}$ & $\mathrm{HF}$ & UN(1) & UN & UNR & TOEP(2) & AR(1) \\
\hline Bal. $^{1}$ & Dias & 11,84 & 25,76 & 99,44 & 34,44 & 1350,76 & 1517,84 & 58,00 & 37,88 \\
\hline Des. $^{2}$ & Dias & 16,56 & 21,40 & 112,36 & 32,16 & 1088,24 & 1206,88 & 57,84 & 31,92 \\
\hline Bal. & Trat*Dias & 17,12 & 18,16 & 109,32 & 29,76 & 1296,44 & 1251,68 & 59,04 & 49,76 \\
\hline Des. & Trat*Dias & 25,68 & 18,12 & 167,16 & 35,52 & 1134,36 & 1234,20 & 44,44 & 46,76 \\
\hline
\end{tabular}

Dessa forma, observando-se os resultados da Tabela 19, conclui-se que para os casos simulados a partir da distribuição normal, somente para as estruturas da matriz de covariâncias VC e CS a hipótese de aderência dos níveis mínimos de significância à distribuição uniforme não foram rejeitadas, tanto para os casos de dados balanceados como para os desbalanceados. Para a matriz UN(1) os valores da estatística $\chi^{2}$ ficaram próximos da região de aceitação e somente para a interação Tratamentos $\times$ Dias, no caso balanceado, o teste de aderência não foi rejeitado. Porém, 
para as demais estruturas da matriz de covariâncias a acurácia da análise de variância não foi satisfatória.

Um detalhe importante são os resultados obtidos para a estrutura HF, da condição de H-F, pois os dados gerados a partir dessa matriz de covariâncias não produziram resultados satisfatórios quanto à acurácia da análise de variância. Esse fato chama a atenção, pois essa estrutura da matriz de covariâncias é uma condição necessária e suficiente para que os resultados do teste $F$ para os fatores da subparcela (intraindivíduos) sejam válidos. 
Tabela 20. Valores da Estatística $\chi^{2}$ para o teste de aderência da distribuição de frequêencia dos níveis mínimos de significância associados aos valores da estatística $F$ para Dias $\mathrm{e}$ interação Tratamentos $\times$ Dias, com dados balanceados e desbalanceados, para a distribuição normal contaminada.

\begin{tabular}{|c|c|c|c|c|c|c|c|c|c|}
\hline \multicolumn{10}{|c|}{$=0,05$ e $(1-\alpha)=0$} \\
\hline & \multirow{2}{*}{$\begin{array}{c}\text { Estatística } \\
\mathbf{F}\end{array}$} & \multicolumn{8}{|c|}{ Estruturas da matriz de covariâncias } \\
\hline & & VC & CS & HF & $\mathrm{UN}(1)$ & UN & UNR & TOEP(2) & $\operatorname{AR}(1)$ \\
\hline Bal. & Dias & 13,60 & 19,52 & 33,28 & 104 & & 24,24 & 16,04 & 32,40 \\
\hline Des. & Dias & 24,12 & 24,60 & 42,04 & 33,80 & 68 & 88 & 34,84 & 29,40 \\
\hline Bal. & Trat*Dias & 11,20 & 8,00 & 33,96 & 14,72 & 38 & 50 & 51,00 & 57,36 \\
\hline Des. & Trat*Dias & 22,72 & 21,08 & 39,36 & 27,20 & & & 48,60 & 33,08 \\
\hline \multicolumn{10}{|c|}{$\alpha=0,10$ e $(1-\alpha)=0,90$} \\
\hline & \multirow{2}{*}{$\begin{array}{c}\text { Estatística } \\
\mathrm{F} \\
\end{array}$} & \multicolumn{8}{|c|}{ Estruturas da matriz de covariâncias } \\
\hline & & VC & CS & $\mathrm{HF}$ & $\mathrm{UN}(1)$ & UN & UNR & TOEP(2) & AR(1) \\
\hline Bal. & Dias & 17,16 & 8,60 & 30,52 & & & & 20,60 & \\
\hline Des. & Dias & 13,00 & 19,00 & 46,32 & & & & 16 & $\underline{0}$ \\
\hline Bal. & Trat*Dias & 11,80 & 21,20 & 45,12 & 37 & & & 47,72 & 64 \\
\hline Des. & Trat*Dias & 10,12 & 10,32 & 81,36 & 34,32 & 1008,96 & & 32,60 & 26, \\
\hline \multicolumn{10}{|c|}{$\alpha=0,15$ e $(1-\alpha)=0,85$} \\
\hline & \multirow{2}{*}{$\begin{array}{c}\text { Estatística } \\
\mathbf{F}\end{array}$} & \multicolumn{8}{|c|}{ Estruturas da matriz de covariâncias } \\
\hline & & $\mathrm{VC}$ & $\mathrm{CS}$ & HF & $\mathrm{UN}(1)$ & $\mathbf{U N}$ & UNR & TOEP(2) & AR(1) \\
\hline Bal. & Dias & 12,52 & 21,28 & 30,48 & & & & 23,44 & 23,08 \\
\hline Des. & Dias & 23,60 & 26,72 & 16,12 & & & & 43,80 & 88 \\
\hline Bal. & & & 26,56 & 37,28 & & & & 44,56 & 32 \\
\hline Des. & Trat*Dias & 26,24 & 15,16 & 50,52 & 40,20 & 1009,80 & 790,96 & 28,36 & 23,04 \\
\hline \multicolumn{10}{|c|}{$\alpha=0,20$ e $(1-\alpha)=0,80$} \\
\hline & Estatística & \multicolumn{8}{|c|}{ Estruturas da matriz de covariâncias } \\
\hline & $\mathbf{F}$ & $\mathrm{VC}$ & $\mathrm{CS}$ & $\mathrm{HF}$ & $\mathrm{UN}(1)$ & UN & UNR & TOEP(2) & $\operatorname{AR}(1)$ \\
\hline Bal. & Dias & 15,52 & 26,38 & 51,52 & & 8 & 34 & 46,40 & 30,24 \\
\hline Des. & Dias & 22,32 & 13,40 & 27,88 & & 48 & 80 & 32,64 & 31,16 \\
\hline Bal. & Trat & 10,60 & 12,40 & 43,04 & & & & 95,40 & \\
\hline Dac & Trat* & 12,60 & 17,12 & 35,44 & 23,96 & 935,28 & 785,64 & 41,00 & 29,28 \\
\hline
\end{tabular}

Casos simulados a partir da distribuição normal contaminada, encontrados na Tabela 20, mostram que para as matrizes VC e CS, com as ponderações $\alpha=0,05$, $0,10,0,15$ e 0,20 , a hipótese de aderência dos níveis mínimos de significância à distribuição uniforme não foi rejeitada, tanto para os casos de dados balanceados como desbalanceados. 
Observando-se os resultados da estrutura $\mathrm{HF}$, verifica-se que os valores de $\chi^{2}$ ficaram mais próximos da região de aceitação e em alguns casos como $\alpha=0,15 \mathrm{e}$ 0,20 para o fator Dias com desbalanceamento, a hipótese de aderência não foi rejeitada.

Para a estrutura UN(1) a acurácia da análise de variância é satisfatória quando $\alpha=0,05$ e 0,20 .

As estruturas UN e UNR não apresentaram, em nenhuma ocasião, a indicação de acurácia satisfatória da análise de variância.

A estrutura TOEP(2) $\operatorname{com} \alpha=0,05,0,10$ e 0,15 não rejeitou a hipótese de aderência para o fator Dias com desbalanceamento e com $\alpha=0,15$ para o fator Tratamentos $\times$ Dias com desbalanceamento.

A estrutura $\operatorname{AR}(1)$, apresentou acurácia da análise, ou seja, a hipótese de aderência não foi rejeitada quando $\alpha=0,05$ e 0,10 para $o$ fator Dias com desbalanceamento, e para $\alpha=0,15$ para o fator Dias com balanceamento, e com $\alpha=$ 0,15 e 0,20 para a interação Tratamentos $\times$ Dias com desbalanceamento.

Dessa forma, somente as matrizes VC e CS, que satisfazem à condição de esfericidade (condição de H-F), apresentaram acurácia satisfatória da análise de variância, tanto para os dados simulados com a distribuição normal, como para a distribuição normal contaminada.

Saliente-se ainda que no caso da distribuição normal os valores da estatística $\chi^{2}$ foram maiores (mais distantes da região de aceitação).

Com relação aos dados provenientes da distribuição normal contaminada as estruturas $U N(1)$, TOEP(2) e $\operatorname{AR}(1)$ em algumas situações não rejeitaram a hipótese de aderência dos níveis mínimos de significância à distribuição uniforme.

Para os casos considerados com efeitos não nulos, as frequências observadas dos níveis mínimos de significância foram dispostos nas classes de frequências $(0,00-0,05],(0,05-0,10],(0,10-0,15]$ e $(0,15-0,20]$, e são apresentados nas Tabelas $21,22,23,24$ e 25. Foram consideradas essas classes de frequências por se 
tomarem decisões sobre testes de hipóteses baseando-se em um nível de significância menor do que 0,20 .

Tabela 21. Frequências dos níveis mínimos de significância nas primeiras classes, associados aos valores da estatística $F$ para Dias $e$ interação Tratamentos $\times$ Dias, com dados balanceados e desbalanceados, para a distribuição normal.

\begin{tabular}{ccccc|c|cccc}
\hline & & \multicolumn{4}{c|}{ Dias } & \multicolumn{4}{c}{ Tratamentos $\times$ Dias } \\
\cline { 3 - 10 } & Estrutura & \multicolumn{4}{c|}{ Classes } & \multicolumn{4}{c}{ Classes } \\
\cline { 2 - 11 } & de $\Sigma$ & $0,00-0,05$ & $0,05-0,10$ & $0,10-0,15$ & $0,15-0,20$ & $0,00-0,05$ & $0,05-0,10$ & $0,10-0,15$ & $0,15-0,20$ \\
\hline & VC & 999 & 0 & 0 & 0 & 999 & 0 & 0 & 0 \\
B & CS & 999 & 0 & 0 & 0 & 999 & 0 & 0 & 0 \\
A & HF & 999 & 0 & 0 & 0 & 941 & 30 & 14 & 6 \\
L & UN(1) & 999 & 0 & 0 & 0 & 992 & 4 & 1 & 0 \\
A & UN & 999 & 0 & 0 & 0 & 426 & 82 & 53 & 55 \\
N & UNR & 999 & 0 & 0 & 0 & 424 & 78 & 64 & 38 \\
C. & TOEP(2) & 999 & 0 & 0 & 0 & 989 & 7 & 0 & 0 \\
& AR(1) & 999 & 0 & 0 & 0 & 996 & 1 & 0 & 0 \\
\hline & VC & 999 & 0 & 0 & 0 & 994 & 4 & 0 & 0 \\
D & CS & 999 & 0 & 0 & 0 & 998 & 0 & 0 & 0 \\
E & HF & 999 & 0 & 0 & 0 & 935 & 38 & 10 & 6 \\
S & UN(1) & 999 & 0 & 0 & 0 & 991 & 5 & 1 & 0 \\
B & UN & 999 & 0 & 0 & 0 & 415 & 70 & 60 & 41 \\
A & UNR & 999 & 0 & 0 & 0 & 432 & 69 & 48 & 35 \\
L. & TOEP(2) & 999 & 0 & 0 & 0 & 985 & 10 & 0 & 0 \\
& AR(1) & 999 & 0 & 0 & 0 & 990 & 4 & 0 & 1 \\
\hline
\end{tabular}


Tabela 22. Frequências dos níveis mínimos de significância nas primeiras classes, associados aos valores da estatística $F$ para Dias $e$ interação Tratamentos $\times$ Dias, com dados balanceados e desbalanceados, para a distribuição normal contaminada com $\alpha=0,05$ e $(1-\alpha)=0,95$.

\begin{tabular}{cccccc|ccccc}
\hline & & \multicolumn{5}{c|}{ Dias } & \multicolumn{4}{c}{ Tratamentos $\times$ Dias } \\
\cline { 3 - 10 } & Estrutura & \multicolumn{4}{c}{ Classes } & \multicolumn{4}{c}{ Classes } \\
\cline { 2 - 10 } & de $\Sigma$ & $\mathbf{0 , 0 0 - 0 , 0 5}$ & $0,05-0,10$ & $0,10-0,15$ & $0,15-0,20$ & $0,00-0,05$ & $0,05-0,10$ & $0,10-0,15$ & $0,15-0,20$ \\
\hline & VC & 999 & 0 & 0 & 0 & 932 & 28 & 13 & 8 \\
B & CS & 999 & 0 & 0 & 0 & 945 & 34 & 8 & 3 \\
A & HF & 999 & 0 & 0 & 0 & 822 & 77 & 23 & 25 \\
L & UN(1) & 999 & 0 & 0 & 0 & 815 & 90 & 35 & 22 \\
A & UN & 999 & 0 & 0 & 0 & 412 & 78 & 62 & 44 \\
N & UNR & 999 & 0 & 0 & 0 & 412 & 80 & 64 & 45 \\
C. & TOEP(2) & 999 & 0 & 0 & 0 & 922 & 32 & 9 & 7 \\
& AR(1) & 999 & 0 & 0 & 0 & 943 & 23 & 10 & 5 \\
\hline & VC & 999 & 0 & 0 & 0 & 925 & 45 & 11 & 8 \\
D & CS & 999 & 0 & 0 & 0 & 930 & 37 & 16 & 5 \\
E & HF & 999 & 0 & 0 & 0 & 807 & 78 & 41 & 27 \\
S & UN(1) & 999 & 0 & 0 & 0 & 823 & 80 & 36 & 23 \\
B & UN & 999 & 0 & 0 & 0 & 410 & 77 & 50 & 42 \\
A & UNR & 999 & 0 & 0 & 0 & 391 & 86 & 54 & 48 \\
L. & TOEP(2) & 999 & 0 & 0 & 0 & 878 & 45 & 20 & 21 \\
& AR(1) & 999 & 0 & 0 & 0 & 933 & 33 & 15 & 2 \\
\hline
\end{tabular}

Tabela 23. Frequências dos niveis mínimos de significância nas primeiras classes, associados aos valores da estatística $F$ para Dias e interação Tratamentos $\times$ Dias, com dados balanceados e desbalanceados, para a distribuição normal contaminada $\operatorname{com} \alpha=0,10$ e $(1-\alpha)=0,90$.

\begin{tabular}{cccccc|ccccc}
\hline & & \multicolumn{5}{c|}{ Dias } & \multicolumn{4}{c}{ Tratamentos $\times$ Dias } \\
\cline { 2 - 10 } & Estrutura & \multicolumn{4}{c|}{ Classes } & \multicolumn{4}{c}{ Classes } \\
\cline { 2 - 10 } & de $\Sigma$ & $0,00-0,05$ & $0,05-0,10$ & $0,10-0,15$ & $0,15-0,20$ & $0,00-0,05$ & $0,05-0,10$ & $0,10-0,15$ & $0,15-0,20$ \\
\hline & VC & 999 & 0 & 0 & 0 & 956 & 25 & 7 & 1 \\
B & CS & 999 & 0 & 0 & 0 & 964 & 25 & 3 & 2 \\
A & HF & 999 & 0 & 0 & 0 & 865 & 60 & 26 & 19 \\
L & UN(1) & 999 & 0 & 0 & 0 & 868 & 75 & 21 & 12 \\
A & UN & 999 & 0 & 0 & 0 & 441 & 85 & 58 & 51 \\
N & UNR & 999 & 0 & 0 & 0 & 457 & 94 & 46 & 41 \\
C. & TOEP(2) & 999 & 0 & 0 & 0 & 949 & 23 & 11 & 2 \\
& AR(1) & 999 & 0 & 0 & 0 & 967 & 15 & 4 & 2 \\
\hline & VC & 999 & 0 & 0 & 0 & 946 & 30 & 9 & 4 \\
D & CS & 999 & 0 & 0 & 0 & 968 & 19 & 6 & 1 \\
E & HF & 999 & 0 & 0 & 0 & 864 & 63 & 25 & 15 \\
S & UN(1) & 999 & 0 & 0 & 0 & 877 & 58 & 25 & 12 \\
B & UN & 999 & 0 & 0 & 0 & 404 & 87 & 64 & 46 \\
A & UNR & 999 & 0 & 0 & 0 & 463 & 101 & 58 & 41 \\
L. & TOEP(2) & 999 & 0 & 0 & 0 & 927 & 32 & 16 & 5 \\
& AR(1) & 999 & 0 & 0 & 0 & 949 & 28 & 6 & 4 \\
\hline
\end{tabular}


Tabela 24. Frequências dos níveis mínimos de significância nas primeiras classes, associados aos valores da estatística $F$ para Dias e interação Tratamentos $\times$ Dias, com dados balanceados e desbalanceados, para a distribuição normal contaminada com $\alpha=0,15$ e $(1-\alpha)=0,85$.

\begin{tabular}{cccccc|ccccc}
\hline & & \multicolumn{4}{c|}{ Dias } & \multicolumn{4}{c}{ Tratamentos $\times$ Dias } \\
\cline { 2 - 10 } & Estrutura & \multicolumn{3}{c}{ Classes } & \multicolumn{4}{c}{ Classes } \\
\cline { 2 - 10 } & de $\Sigma$ & $0,00-0,05$ & $0,05-0,10$ & $0,10-0,15$ & $0,15-0,20$ & $0,00-0,05$ & $0,05-0,10$ & $0,10-0,15$ & $0,15-0,20$ \\
\hline & VC & 999 & 0 & 0 & 0 & 969 & 14 & 5 & 3 \\
B & CS & 999 & 0 & 0 & 0 & 984 & 10 & 1 & 0 \\
A & HF & 999 & 0 & 0 & 0 & 901 & 45 & 25 & 10 \\
L & UN(1) & 999 & 0 & 0 & 0 & 913 & 44 & 19 & 9 \\
A & UN & 999 & 0 & 0 & 0 & 456 & 86 & 54 & 57 \\
N & UNR & 999 & 0 & 0 & 0 & 460 & 109 & 55 & 49 \\
C & TOEP(2) & 999 & 0 & 0 & 0 & 965 & 17 & 5 & 2 \\
& AR(1) & 999 & 0 & 0 & 0 & 975 & 13 & 0 & 4 \\
\hline & VC & 999 & 0 & 0 & 0 & 964 & 18 & 9 & 0 \\
D & CS & 999 & 0 & 0 & 0 & 974 & 18 & 1 & 0 \\
E & HF & 999 & 0 & 0 & 0 & 902 & 48 & 24 & 7 \\
S & UN(1) & 999 & 0 & 0 & 0 & 923 & 37 & 20 & 6 \\
B & UN & 999 & 0 & 0 & 0 & 465 & 83 & 62 & 51 \\
A & UNR & 999 & 0 & 0 & 0 & 472 & 84 & 63 & 43 \\
L. & TOEP(2) & 999 & 0 & 0 & 0 & 961 & 20 & 3 & 4 \\
& AR(1) & 999 & 0 & 0 & 0 & 966 & 17 & 9 & 2 \\
\hline
\end{tabular}

Tabela 25. Frequências dos níveis mínimos de significância nas primeiras classes, associados aos valores da estatística $F$ para Dias $e$ interação Tratamentos $\times$ Dias, com dados balanceados e desbalanceados, para a distribuição normal contaminada $\operatorname{com} \alpha=0,20$ e $(1-\alpha)=0,80$.

\begin{tabular}{cccccc|ccccc}
\hline & & \multicolumn{4}{c|}{ Dias } & \multicolumn{4}{c}{ Tratamentos $\times$ Dias } \\
\cline { 2 - 10 } & Estrutura & \multicolumn{3}{c}{ Classes } & \multicolumn{4}{c}{ Classes } \\
\cline { 2 - 10 } & de $\Sigma$ & $0,00-0,05$ & $0,05-0,10$ & $0,10-0,15$ & $0,15-0,20$ & $0,00-0,05$ & $0,05-0,10$ & $0,10-0,15$ & $0,15-0,20$ \\
\hline & VC & 999 & 0 & 0 & 0 & 986 & 5 & 3 & 0 \\
B & CS & 999 & 0 & 0 & 0 & 988 & 6 & 2 & 0 \\
A & HF & 999 & 0 & 0 & 0 & 942 & 28 & 14 & 2 \\
L & UN(1) & 999 & 0 & 0 & 0 & 946 & 22 & 15 & 5 \\
A & UN & 999 & 0 & 0 & 0 & 493 & 77 & 58 & 41 \\
N & UNR & 999 & 0 & 0 & 0 & 522 & 85 & 45 & 38 \\
C. & TOEP(2) & 999 & 0 & 0 & 0 & 983 & 12 & 2 & 0 \\
& AR(1) & 999 & 0 & 0 & 0 & 983 & 7 & 4 & 0 \\
\hline & VC & 999 & 0 & 0 & 0 & 987 & 5 & 2 & 1 \\
D & CS & 999 & 0 & 0 & 0 & 994 & 4 & 0 & 0 \\
E & HF & 999 & 0 & 0 & 0 & 943 & 27 & 11 & 2 \\
S & UN(1) & 999 & 0 & 0 & 0 & 934 & 34 & 16 & 3 \\
B & UN & 999 & 0 & 0 & 0 & 497 & 99 & 59 & 50 \\
A & UNR & 999 & 0 & 0 & 0 & 487 & 81 & 49 & 43 \\
L. & TOEP(2) & 999 & 0 & 0 & 0 & 976 & 11 & 6 & 0 \\
& AR(1) & 999 & 0 & 0 & 0 & 980 & 11 & 4 & 0 \\
\hline
\end{tabular}


Através das Tabelas 21, 22, 23, 24 e 25 observa-se que independentemente do tipo da distribuição, se normal ou normal contaminada, bem como do balanceamento ou desbalanceamento dos dados, com os efeitos utilizados, em todos os casos das estruturas da matriz de covariâncias, o teste superestima a indicação de efeitos, quando esses existem. Isso tanto para os testes do fator Dias, como para a interação Tratamentos $\times$ Dias.

Para os níveis mínimos de significância do fator Dias, em nenhum dos casos as frequências $(0,05-0,10],(0,10-0,15]$ e $(0,15-0,20]$ apresentaram uma observação sequer. Isso se deve ao fato de que os efeitos utilizados apresentam muita diferença. Pode-se verificar isso observando os efeitos não nulos para o fator Dias que são $\tau_{1}=-$ $9,945, \tau_{2}=-7,288, \tau_{3}=-4,320, \tau_{4}=-2,988, \tau_{5}=-0,748, \tau_{6}=0$, por exemplo, tem-se $-9,945$ para o primeiro tempo e zero para o tempo 6 .

Malheiros (1999), que trabalhou com diferentes estruturas de covariâncias, considerando, porém, que todas as estruturas tinham a mesma variância, diferindo somente as covariâncias, e apresentando efeitos dos parâmetros pequenos, obteve resultados em que as matrizes de covariâncias sem estruturada com correlações linearmente crescentes e decrescentes apresentaram acurácia satisfatória para a análise univariada. Também encontrou estruturas em que o teste não superestima a indicação dos efeitos quando eles existem.

Pode-se, então, concluir que dependendo dos efeitos dos parâmetros que o experimento apresenta, estes influenciam o resultado dos testes no sentido de superestimar a indicação dos efeitos, quando esses efeitos têm o intervalo de variação grande, e também quando as estruturas da matriz de covariâncias utilizadas não apresentam variâncias iguais.

Para esse caso em que as estruturas das matrizes de covariâncias para as simulações não apresentaram variâncias iguais e o intervalo de variação dos efeitos é grande, conclui-se que a análise de variância univariada só apresenta resultados válidos para as estatísticas $\mathrm{F}$ dos fatores intra-indivíduos, se a matriz de covariâncias atender à condição de esfericidade. Caso a matriz não atenda a essa condição, correções deverão 
ser utilizadas para os números de graus de liberdade dos fatores intra-indivíduos, ou então, optar por modelos multivariados ou modelos mistos. 


\section{CONCLUSÕES}

De acordo com as metodologias empregadas neste trabalho, e com base nos resultados obtidos, pode-se chegar às seguintes conclusões:

a) Com relação aos procedimentos do SAS, proc GLM e proc MIXED, pode-se concluir que ambos são de grande valia na análise de medidas repetidas. O proc GLM é mais limitado por não permitir que se trabalhe com dados desbalanceados, mas para a análise univariada e multivariada apresenta a maioria dos testes usuais implementados. O proc MIXED tem a vantagem de permitir a utilização de dados desbalanceados, a escolha do método de estimação, e para modelos mistos, apresenta várias estruturas de covariâncias já implementadas.

b) As simulações foram importantes por confirmar que a condição de esfericidade é suficiente e necessária para que se tenha uma boa acurácia da análise da variância, para dados de medidas repetidas no tempo, no esquema de parcelas subdivididas (modelo univariado).

c) Os resultados das simulações utilizando a distribuição normal e a normal contaminada, apresentaram resultados semelhantes, ou seja, confirmaram através dos testes de aderência que a utilização de matrizes de covariâncias, que não atendam à condição de esfericidade, levam a resultados inválidos para os testes dos fatores intra-indivíduos. Somente as estruturas VC (componente de variância) e CS (simetria composta) apresentaram acurácia satisfatória para a análise de variância. 
d) Com relação à estrutura H-F (Huynh-Feldt) mais estudos devem ser realizados para se verificar o fato de que dados simulados a partir dessa estrutura, que é uma condição necessária e suficiente, não apresentem resultados razoáveis quanto à acurácia das análises. 


\section{REFERÊNCIAS BIBLIOGRÁFICAS}

BOX, G.E.P. Some theorems on quadratic forms applied in the study of analysis of variance problems I. Effects of inequality of variance in the one-way classification. Annals of the Mathematical Statistics, v.25, p.290-302, 1954.

BOX, G.E.P. Some theorems on quadratic forms applied in the study of analysis of variance problems II. Effects of inequality of variance and of correlation between erros in the two-way classification. Annals of the Mathematical Statistics, v.25, p.484-498, 1954.

BOSWELL, M.T.; GORE, S.D.; PATIL, G.P.; et al. The art of computer generation of random variables. In: RAO, C.R. (Ed.) Computacional statistics. North-Holland: Elsevier Science Publishers, 1993. cap.20, p.661-721.

CIÓL, A.M. Métodos de estimação de componentes de variância em modelos mistos. Campinas, 1982. 126p. Dissertação (M.S.) - Universidade Estadual de Campinas

CROWDER, M.J.; HAND, D.J. Analysis of repeated measures. London: Chapman \& Hall, 1990, 256p.

DACHS, N. Estatística computacional. Rio de Janeiro: Livros Técnicos e Científicos Editora, 1988, 236p. 
DIAS, C.T.S. Planejamento de uma fazenda em condições de risco: programação linear e simulação multidimensional. Piracicaba, 1996. 100p. Tese (Doutorado) - Escola Superior de Agricultura "Luiz de Queiroz", Universidade de São Paulo.

DIGGLE, P.J. An approach to the analysis of repeated measurements. Biometrics, v.44, p.959-971, 1988.

DIGGLE, P.J.; LIANG, K-Y.; ZEGER, S.L. Analysis of longitudinal data. 4.ed New York : Oxford University Press Inc., 1998, 253p. (Oxford Statistical Science Series 13).

FERNANDEZ, G.C.J. Repeated measure analysis of line-source sprinkler experiments. HortScience, v.26, n.4, p.339-342, 1991.

GEISSER, J.; GREENHOUSE, S.W. An extension of Box's results on the use of the F distribution in multivariate analysis. Annals of the Mathematical Statistics, v.29, p.855-891, 1958.

GUIMARÃES, P.R.B. Modelo linear misto de Laird-Ware; predição de efeitos aleatórios e estimação de parâmetros via filtro de Kalman. Campinas, 1994. 135p. Dissertação (Mestrado) - Universidade Estadual de Campinas.

HARVILLE, D.A. Bayesian inference for variance components using only contrasts. Biometrika, v.61, p.383-385, 1974.

HUYNH, H.; FELDT, L.S. Conditions under which mean square rations in repeated measurements designs have exact F-distributions. Journal of the American Statistical Association, v.65, n.332, p.1582-1589, Dez 1970. 
HUYNH, H.; FELDT, L.S. Estimation of the Box correction for degrees of freedom from sample data in the randomized block and split-plot designs. Journal of Educational Statistics, v.1, n. 1, p.69-82, 1976.

HUYNH, H.; FELDT, L.S. Some aproximate tests for repeated measurement designs. Psychometrika, v.43, n.2, p.161-175, Jun 1978.

JENNRICH, R.I.; SCHLUCHTER, M.D. Unbalanced repeated measures models with structured covariance matrices. Biometrics, v.42, p.805-820, Dez 1986.

JOHNSON, M.E. Multivariate statistical simulation. New York: John Wiley and Sons, 1987, 230p.

KANASHIRO, S. Efeitos de diferentes substratos na produção da espécie Aechmea fasciata (Lindley) baker em vasos. Piracicaba, 1999. 79p. Dissertação (Mestrado) Escola Superior de Agricultura "Luiz de Queiroz", Universidade de São Paulo.

KIRK, R.E. Experimental design: procedures for the behavioral. 1995, cap.6, p.243-267: Randomized block designs.

KOCH, G.G; ELASSHOFF, J.D.; AMARA, I.A. Repeated measures studies: design and analysis. In: JOHNSON, N.L.; KOTZ, S. Encyclopedia of Statistical Sciences. New York: Jonh Wiley and Sons Inc., 1985, p.46-71

KSHIRSAGAR, A.M.; SMITH, W.B. Growth Curves. New York: Marcel Dekker Inc., 1995, 359p.

KUEHL, R.D. Statistical Principles of Research design and analysis duxbury press. Belmont California, 1994. cap. 15, p.499-528: Repeated measures designs. 
LAIRD, N.M.; WARE, J.H. Random-effects models for longitudinal data. Biometrics, v.38, p.963-974, 1982.

LIMA, C.G. Análise de dados longitudinais provenientes de experimentos em blocos casualizados. Piracicaba, 1996. 126p. Tese (Doutorado) - Escola Superior de Agricultura “'Luiz de Queiroz”, Universidade de São Paulo.

LITTELL, R.C.; HENRY, P.R.; AMMERMAN, C.B. Statistical analysis of repeated measures data using SAS procedures. American Society of Animal Science, v.76, p.1216-1231, 1998.

MALHEIROS, E.B. Precisão da análise de dados longitudinais, com diferentes estruturas para a matriz de variâncias e covariâncias, quando se utiliza o esquema em parcelas subdivididas. Revista de Matemática e Estatística, v.17, p.229-239, 1999.

MATSUSHITA, R.Y. Modelos longitudinais mistos com correlação serial nos erros. Campinas, 1994. 188p. Dissertação (Mestrado) - Universidade Estadual de Campinas.

MAUCHLY, J.W. Significance test for sphericity of a normal n-variate distribution. Annals of Mathematical Statistics, v.11, p.204-209, 1940.

MEREDITH, M.P.; STEHMAN, S.V. Repeated measures experiments in forestry: focus on analysis of response curves. Canadian Journal of Forest Research, v.21, p.957-965, 1991. 
MILLIKEN, G.A.; JOHNSON, D.E. Analysis of messy data v.1 designed experiments. New York: Chapman \& Hall, 1992, 473p.

MOOD, A.M.; GRAYBLL,F.A.; BOES, D.C. Introduction to the theory of statistics. 3.ed. New York: McGraw-Hill, 1974, 564p.

MULLER, K. E. \& BARTON, C. N. Approximate power for repeated-measures ANOVA lacking sphericity. Journal of the American Statistical Association, v.84, n.406, p.549-555, Jun 1989.

SAS/STAT Software: changes and anhancements through release 6.12. SAS Institute Inc. Cary, NC. USA. 1997, 1167p.

SHIMIZU, T. Simulação em computador digital. São Paulo: Edgard Blücher, editora da USP, 1975, 109p.

SINGER, J.M.; ANDRADE, D.F. Análise de dados longitudinais. In: VII SIMPÓSIO NACIONAL DE PROBABILIDADE E ESTATÍSTICA, Campinas, São Paulo, 1986. $106 \mathrm{p}$.

STEEL, R.G.D.; TORRIE, J.H. Principles and procedures of statisitics with special reference to the biological sciences. New York: Mc Graw-Hill Book Company Inc., $1960,481 \mathrm{p}$.

VON ENDE, C.N. Repeated-measures analysis: Growth and other time-dependent measures. In: SCHEINER, S.M.; GUREVITCH, J. (Ed.) Design and analysis of ecological experiments. New York: Chapman and Hall, 1993. cap.6, p.113-137. 
VONESH, F.E.; CHINCHILLI, V.M. Linear and nonlinear models for the analysis of repeated measurements. New York: Marcel Dekker, Inc. 1997. 560p.

WARE, J. H. Linear models for the analysis of longitudinal studies. Amerrican Statistical Association, v.39, n.2, p.95-101, 1985.

WOLFINGER, R. \& CHANG, M. Comparing the SAS GLM and MIXED Procedures for repeated measures. SAS Institute Inc. Cary. NC. www.sas.com/usergroups. (1999). 